\title{
GERMLINE TRANSMISSION OF MITOCHONDRIAL DNA IN THE MOUSE
}

\author{
By \\ Timothy Wai \\ Department of Human Genetics, McGill University, Montreal \\ May 2009
}

A thesis submitted to McGill University in partial fulfillment of the requirements of the degree of Ph.D.

(C) Timothy Wai, 2009 


\section{ACKNOWLEDGEMENTS}

I would like to being by thanking Dr. Eric Shoubridge for his guidance, patience, and unwavering support, especially in the face of personal difficulty. I have greatly benefited from his intelligence and scientific fearlessness and am grateful for his helping during the preparation of this thesis and during my search for a post-doctoral position. I am grateful for the support and continued encouragement of Dr. David Rosenblatt and the assistance provided by Dr. Daniel Dufort and Dr. William Foulkes as members of my supervisory committee. I acknowledge Xiaoyun Zhang and Dr. Asangla Ao, for the biopsy of blastomeres, Dr. Daniel Dufort, for the embryo transfers, and Dr. Daniel Cyr and Mary Gregory, for helping me with sperm analyses, Dr. Nils-Goran Larsson, for the TFAM mice, Dr. Carlos Moraes for the COX10 mice, and Dr. Hans Scholer for the OG2 mice. I am grateful to the staff of the animal care facility for their diligent work and technical assistance.

For the warm and enjoyable atmosphere of the lab I thank Hana Antonicka, Florin Sassarman, Timothy Johns, Tamiko Nishimura, Wan Weraarpachai, Catherine Brunel, Lissi Neiva, and Olga Zurita. Daniella Teoli, Fiona Jones, and Margaret Redpath taught me to teach and, along with Timothy Johns provided valuable technical assistance. Brendan Battersby and Scot Leary, friends and colleagues, both instilled a sense of scientific rigor.

I am grateful for the support of my friends from Montreal and East Van who were invaluable in helping me achieve a healthy balance between work and play. 
Above all, I would like to thank my parents Athinea and Hong Wai, whose love, encouragement, and sacrifices made all my success possible. Pour tout çela, je vous suis reconnaissant. 


\section{Table of Contents}



ABSTRACT

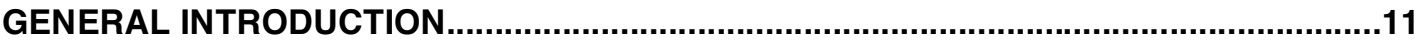

PART 1:THE MITOCHONDRIAL GENOME.........................................14

PART 2: FEMALE GAMETOGENESIS AND EARLY EMBRYOGENESIS............23

PART 3: GERMLINE TRANSMISSION OF MITOCHONDRIAL DNA.................29

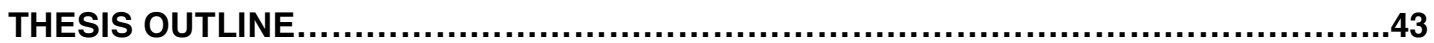

CHAPTER 1: THE MITOCHONDRIAL DNA GENETIC BOTTLENECK RESULTS FROM REPLICATION OF A SUBPOPULATION OF GENOMES DURING OOYCTE MATURATION IN

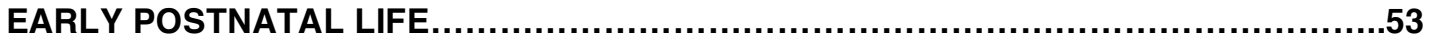

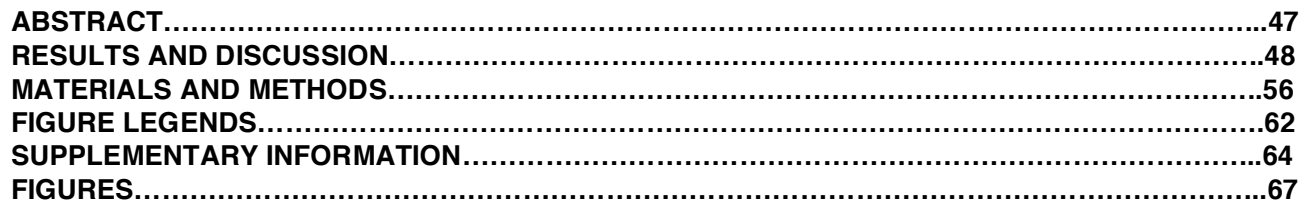

CHAPTER 2: THE SIZE OF THE MITOCHONDRIAL GENETIC BOTTLENECK.............79

ABSTRACT

CHAPTER 3: THE ROLE OF MITOCHONDRIAL DNA COPY NUMBER IN MAMMALIAN

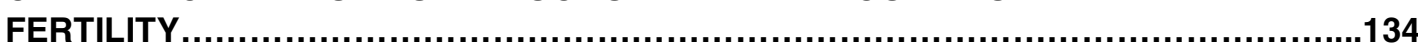

ABSTRACT

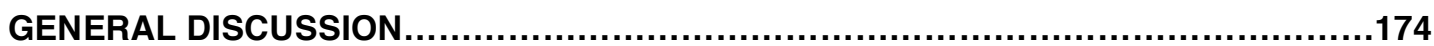

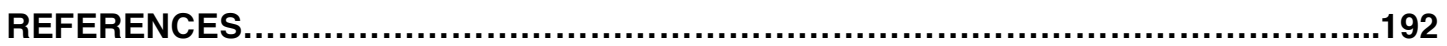






\section{Contributions of Authors}

The general introduction drew upon a book chapter review published in 2007 in Current Topics in Developmental Biology, Vol. 77 Chapter 4, entitled "Mitochondrial DNA and the Mammalian Oocyte" by Dr. Eric A. Shoubridge and Timothy Wai.

Chapter 1 is a manuscript published in 2008 in Nature Genetics, 40 (12): 1484-1488, by Timothy Wai, Daniella Teoli, and Dr. Eric A. Shoubridge. All the experiments in this chapter were designed and executed by Timothy Wai, under the supervision of Dr. Eric A. Shoubridge, with the exception of some DNA extractions from tail biopsy samples and transgene genotyping PCRs, performed by Daniella Teoli.

Chapter 2 is a manuscript to be submitted for publication, by Timothy Wai, Dr. Brendan J. Battersby, and Dr. Eric A. Shoubridge. All the experiments in this chapter were designed and executed by Timothy Wai, with the exception of the generation and genotyping of congenic heteroplasmic mice, which was performed by Dr. Brendan J. Battersby.

Chapter 3 is a manuscript to be submitted for publication, by Timothy Wai, Xiaoyun Zhang, Asangla Ao, Dr. Daniel Dufort, Mary Gregory, Dr. Daniel Cyr, and Dr. Eric A. Shoubridge. Experiments presented in this chapter were designed and executed by Timothy Wai, with the exception of blastomere biopsies, performed by Xiaoyun Zhang and Dr. Asangla Ao, embryo transfers performed by Dr. Daniel Dufort, and sperm analyses performed together with Mary Gregory in the lab of Dr. Daniel Cyr. 


\section{Abbreviations}

2-D: two-dimensional

ATP6 \& 8: subunits of complex 5 of the respiratory chain

BMP4 \& 8b: bone morphogenic protein 4 \& 8b

BrdU: bromodeoxyuridine

COX: subunit of cytochrome c oxidase

CSB: conserved sequence blocks

Ct: Cycle threshold

D-loop: displacement loop of mitochondrial DNA

DNA: deoxyribonucleic acid

Dpc: days post-coitum

EGFP: Enhanced Green Fluorescent Protein

EM: electron microscopy

FACS: Fluorescence Activated Cell Sorting

F1\& F2: first intercross and second intercross

HBSS: Hanks balanced salt solution

HSP: heavy-strand promoter

ICM: inner cell mass

IFITM1, 2, \& 3: Interferon induced transmembrane protein 1, 2, \& 3

kb: kilobase

LHON: Lebers Hereditary Optic Neuropathy

LSP: light-strand promoter

MELAS: mitochondrial encephalomyopathy, lactic acidosis, stoke-like episodes 
MERRF: myoclonus epilepsy, ragged-red fibres

mRNA: messenger ribonucleic acid

mt: mitochondrial

mtDNA: mitochondrial DNA

mtSSB: mitochondrial single stranded binding protein

mtTFB: human mitochondrial transcription factor B

mTERF: mitochondrial transcription termination factor

mtTERM: mitochondrial termination factor

NARP: Neuropathy, Ataxia, and Retinitis Pigmentosa

ND: subunits of complex 1 of the respiratory chain

OCT4: octamer transcription factor, which binds to the ATTTGCAT sequence

$\mathrm{OH}$ : heavy-strand origin of replication

OL: light-strand origin of replication

OXPHOS: oxidative phosphorylation

PALM: photoactivated localization microscope

PBS: phosphate buffered saline

PCR: polymerase chain reaction

PEO: progressive external opthalmoplegia

PGC: primordial germ cell

Phe: Phenylalanine

POLG: DNA polymerase gamma

POU: DNA binding domain found in transcription factors family of the same name Q-PCR: quantitative polymerase chain reaction 
QTL: quantitative trait loci

RFLP: restriction fragment length polymorphism

RITOLS: ribonucleotide incorporation throughout the lagging strand

RNA: ribonucleic acid

RNase MRP: mitochondrial RNA processing endoribonuclease

rRNA: ribosomal ribonucleic acid

STORM: stochastic optical reconstruction microscopy

TEM: transmission electron microscopy

Tfam: mitochondrial transcription factor A

TFB1 \& 2: mitochondrial transcription factor B1 \& B2

TNAP: tissue-nonspecific alkaline phosphatase

Tr: trophectoderm

tRNA: transfer ribonucleic acid

Val: Valine 


\section{Abstract (English)}

In mammals, mitochondria and mitochondrial DNA (mtDNA) are transmitted through the female germline. Mature oocytes contain nearly 200,000 copies of mtDNA, organized at 1-2 copies per organelle. Despite the high genome copy number, mtDNA sequence variants are observed to segregate rapidly between generations, and this has led to the concept of a genetic bottleneck for the transmission of mtDNA. In this thesis, I demonstrate that a subgroup of replicating genomes in the early post-natal ovary is responsible for the rapid segregation of mtDNA. I show that the rate of segregation of mtDNA can be accelerated when mtDNA copy number is further reduced in heteroplasmic germline-specific knockout mouse models, yet very extreme reductions in germ cell mtDNA content seem to cause female-specific infertility. Low copy number embryos can be fertilized and proceed through preimplantation development yet fail to develop normally after implantation. Tracking the developmental outcome of embryos containing a range of mtDNAs points to a developmental threshold of about 50,000 copies of mtDNA in the oocyte In this thesis I advance the hypothesis that the large number of mitochondria and mtDNAs present in the oocyte represent a genetic mechanism to ensure their distribution to the gametes and somatic cells of the next generation. If true, mtDNA copy number may be the most important determinant of oocyte quality not because of the effects on oocyte metabolism, but because too few would result in a maldistribution in the embryo. 


\section{Abstrait (En Français)}

Les mitochondries et l'ADN mitochondrial (ADNmt) sont des organites cellulaires qui ne sont transmis que par l'ovule de la mère. Chez les mammifères, l'ovocyte contient presque 200000 copies d'ADNmt, avec 1-2 copies au sein de chaque mitochondrie. Malgré la grande quantité d'ADNmt, nous observons une ségrégation rapide des variants de séquences entre les générations qui nous amène à l'hypothèse d'un goulot d'étranglement génétique pour l'ADNmt. Par cette thèse, je démontre que ce phénomène est dû à un sous-groupe de génomes mitochondriaux qui se multiplient dans l'ovocyte postnatal de la souris. En outre, je démontre qu'une réduction d'ADNmt dans les cellules de souche germinale peut augmenter la vitesse à laquelle ces génotypes se séparent. Une très importante réduction d'ADNmt dans les ovocytes de ces souris mutantes les rend stériles. Or la fertilisation de ces ovocytes ainsi que le développement préimplantatoire se déroulent normalement, par contre l'embryon qui provient d'un ovocyte avec une très faible quantité d'ADNmt ne peut compléter son développement post-implantatoire. Par cette thèse, je propose l'hypothèse suivante : que la grande quantité de mitochondries et génomes mitochondriaux sert à distribuer un nombre suffisant de ces organites aux cellules somatiques et germinales de la prochaine génération. Si cela est vrai, la quantité d'ADNmt pourrait être le plus important déterminant quant à la qualité de l'ovocyte, pas à cause de ses effets sur le métabolisme de l'ovocyte, mais par le fait qu'une quantité insuffisante empêcherait sa distribution dans les cellules de l'embryon. 


\section{General Introduction}

Professor Lynn Margulis, a pioneer in the field of evolutionary biology, was quoted as saying "Life did not take over the globe by combat, but by networking". As an example, she pointed to the origin of mitochondria, where the symbiosis between ancestral cells and alpha proto-bacteria is thought to have been instrumental in mitochondrial evolution. Several lines of evidence support the idea that both mitochondria and chloroplasts evolved from the endocytosis of bacteria by host cells containing a eukaryotic nucleus, thereby creating endosymbionts. When it was first proposed to explain the origin of chloroplasts (by A.F.W. Schimper in 1883) and then later extended to mitochondria (by I.E Wallin in 1923), the endosymbiont theory was quickly dismissed and ignored but the discovery of DNA within mitochondria (mtDNA), and the morphological resemblance of cyanobacteria to chloroplasts (made possible by the advent of the electron microscope), played pivotal roles in the resurrection of this onceunpopular theory. The discovery of DNA in a cellular compartment outside of the nucleus of eukaryotic cells has proven to be one of the most far-reaching findings in biology, impacting fields from anthropology to zoology and opening up new and exciting avenues of thinking regarding mitochondrial biology in the process.

Yet, conspicuously absent from modern day organellar genomes are the genes present in the proposed descendents of the alpha proto-bacterial ancestor, including a full complement of housekeeping genes. Both the loss and transfer of these genes from the proto-mitochondrial genome to the nucleus have been proposed to account for the present-day configuration of mtDNA. While it is 
curious that only a subset of these genes have been transferred, the remaining mammalian mtDNA genes encode polypeptides that are highly hydrophobic polytopic inner membrane proteins and may therefore be incompatible with the migration to the nuclear genome. Further examination of mitochondrial DNA sequences lead to the discovery that the genetic code used in the expression of mitochondrially-encoded genes often differs from genetic code used in the nucleus. There are several other differences that figure prominently between mitochondrial and nuclear genetics that have since been revealed. Unlike the nuclear genome, mtDNA exists in hundreds to thousands of copies per cell and its replication is not strictly tied to the synthesis of nuclear DNA ${ }^{1}$. Usually, the mtDNA molecules present in a cell, tissue, and organism are identical, which is a condition referred to as homoplasmy. The co-existence of two or more sequence variants (whether neutral or deleterious) is referred to as heteroplasmy and in the context of mutations in mtDNA, such as those that can cause mitochondrial diseases, heteroplasmy often refers to the mutant load, which can vary between tissues of an individual. Such mutations can only be passed on from mother to offspring because the early embryo inherits all its mitochondria from the oocyte. With very few exceptions, uniparental transmission of mtDNA has been evolutionary conserved in all eukaryotes, perhaps due to the disparity in cytoplasmic volume between the male and female gametes. In most metazoans in which it has been studied, the increase in cytoplasmic volume that occurs during female gametogenesis is accompanied by an increase in mitochondrial number, culminating in a mature female gamete harbouring hundreds of 
thousands of mitochondria and mtDNAs ${ }^{2}$. The mtDNA copy number in the mammalian oocyte is orders of magnitude greater than in the sperm. Despite the high copy number in the mammalian oocyte, rapid segregation of mtDNA has been observed in heteroplasmic animal models as well as in human pedigrees. An intergenerational mitochondrial genetic bottleneck was proposed to explain the segregation patterns observed in offspring born to heteroplasmic mothers ${ }^{3}$, such as the heteroplasmic mice used to prove that the rapid segregation of mtDNA in the female germline of the mouse is due to random genetic drift ${ }^{4}$. While this study could not definitively pinpoint the genetic bottleneck to a particular stage of germline development, a working hypothesis placed the genetic bottleneck at the outset of embryonic germline development, when the cellular content of mitochondria was thought to be much lower than in the mature oocytes. This hypothesis gained widespread acceptance and remained unchallenged for over a decade ${ }^{4}$. However, recent reports scrutinizing this model have failed to shed further light on the rules of transmission governing the segregation of $m t D N A^{5,6}$.

The aim of this thesis is to describe the mitochondrial genetic bottleneck that is responsible for the rapid segregation of mtDNA in the female germline, which requires an explanation of both the mitochondrial genome (Part 1) as well as the process of female gametogenesis (Part 2). I will conclude by describing what is known about the germline transmission of mtDNA (Part 3) before clearly defining the individual aims of the thesis. 


\section{Part I: The Mitochondrial Genome:}

\section{Mitochondrial DNA Structure}

MtDNA is a double-stranded circular DNA molecule of $\sim 16.5 \mathrm{~kb}$ in all

mammals in which it has been sequenced (Figure 1). The two stands are referred to as heavy $(\mathrm{H})$ and light $(\mathrm{L})$ reflecting their behaviour in alkaline cesium chloride density gradients due to a guanine $(\mathrm{G})$ and thymidine $(\mathrm{T})$ content bias of the $\mathrm{H}$ strand. Mammalian mtDNA codes for 13 polypeptides (Complex I subunits: ND1, ND2, ND3, ND4, ND4L, ND5, ND6; Complex III subunits: Cytochrome b, Complex IV, COI, COII, COIII, Complex V: ATP6, ATP8), all of which are subunits of the enzyme complexes of the oxidative phosphorylation system, and 22 transfer RNAs (tRNAs) and 2 ribosomal RNAs (rRNAs), which constitute part of the dedicated mitochondrial translation machinery. Interestingly, the $\mathrm{H}$ strand encodes for the majority of these genes, while the $L$ strand encodes only 8 tRNAs and one protein-coding gene (ND6). The genome is exceedingly compact; there are no introns, and there is only one non-coding (control) region of $\sim 1 \mathrm{~kb}$ that contains the replication origin for leading strand synthesis $\left(\mathrm{O}_{\mathrm{H}}\right)$, and the promoters for transcription of the $\mathrm{H}$ - and L-strands. This region, known as the displacement loop (D-loop), is highly polymorphic and shows little conservation throughout vertebrate evolution, except for sequence elements that have been shown to be of importance during both replication and transcription, known as conserved sequence blocks (CSB) I, II, and III. 
Figure 1

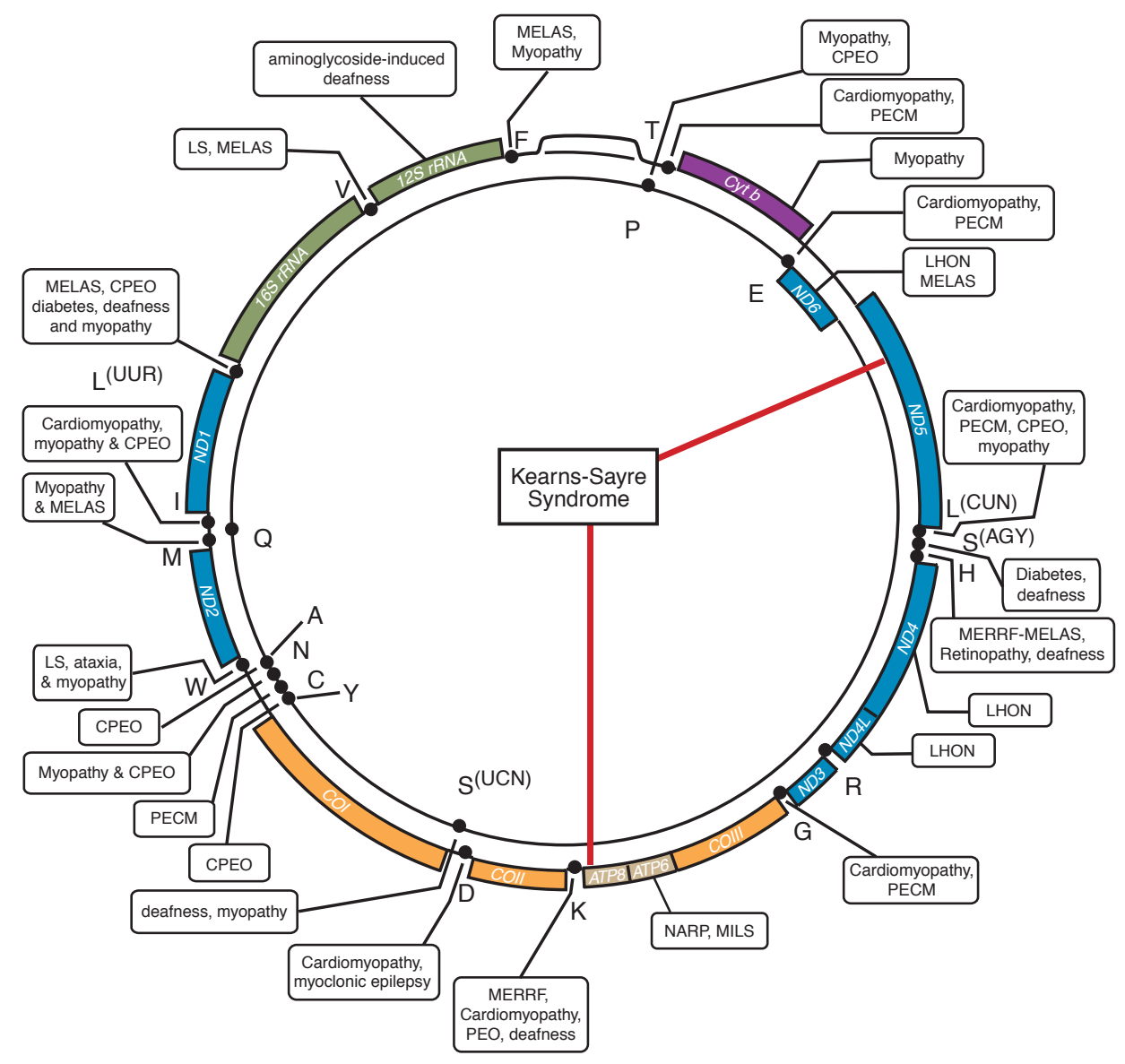




\section{Mitochondrial DNA Replication}

The mechanism of mtDNA replication has been the subject of recent controversy. Mammalian cells grown in tissue culture have provided a convenient platform for the investigation of the replication of mitochondrial DNA. Most of what is known regarding the mechanism, dynamics, and kinetics of replication has been extrapolated from studies performed in this system.

The conventional, strand-asynchronous displacement model developed over the past two decades by Clayton and colleagues ${ }^{7,8}$ was suggested to be an artifact of biased incorporation of ribonucleotides into the L-strand ${ }^{9}$, and a coupled, strand-synchronous mechanism was proposed instead by Holt and colleagues ${ }^{10,11}$. In the strand displacement model, leading strand synthesis of the $\mathrm{H}$-strand starts from $\mathrm{O}_{\mathrm{H}}$ in the control region and proceeds about 2/3 the way around the molecule until a second origin $\left(\mathrm{O}_{\mathrm{L}}\right)$ is exposed, allowing synthesis of the L-strand to proceed. Hence, the synthesis of the L-strand initiated from $\mathrm{O}_{\mathrm{L}}$ is absolutely contingent upon its displacement by the nascent DNA being synthesized from the $\mathrm{H}$-strand. This mechanism of DNA synthesis produces two daughter genomes, one with a parental $\mathrm{H}$-strand and the other with the parental L-strand.

The alternative model, based largely on evidence from two-dimensional (2D) agarose gels, proposes that replication is strand-coupled, originating from a broad zone around $\mathrm{O}_{\mathrm{H}}{ }^{11}$. A recent study in which atomic force microscopy was used to examine mtDNA replicative intermediates in mouse liver was entirely consistent with the strand-displacement model, and did not show any evidence of 
the $\theta$ structures that would be predicted from the strand-coupled model ${ }^{12}$. The same study also provided evidence for alternative L-strand origins, and it was suggested that their presence, in addition to the propensity for branch migration in replicating DNA, might account for the patterns observed by 2-D gel electrophoresis that were interpreted as evidence for strand-coupled replication ${ }^{13}$. More recently, proponents of the strand-coupled model demonstrated the presence of ribonucleotide incorporation throughout the lagging strand (termed RITOLS). Conceding to the existence of the conventional strand-displacement model under certain conditions, Holt and coworkers proposed that the majority of mtDNA replication occurs via a strand-coupled model, based on the observed propensity of RITOLS intermediates in vertebrate tissues ${ }^{14}$. Clearly, this area remains one of continued controversy and so it would be imprudent to conclusively declare either model as being exclusively correct at this time ${ }^{15}$.

Replication is catalyzed by a distinct nuclear-encoded polymerase, the $Y$ DNA polymerase, which possesses similarities to bacteriophage homologues. This enzyme complex is composed of a large catalytic subunit (POLG), which is responsible for the 5'-3' polymerization as well as the 3'-5' exonuclease activity. A smaller accessory subunit (POLG2), present as a homodimer, binds to the RNA primer and stimulates the processivity of the catalytic subunit ${ }^{16,17}$. Both replication models require a primase to prime replication of the L-strand, and although a primase activity has been recovered from human mitochondria ${ }^{18}$, its molecular identity remains unknown. Phylogenetic analysis of eukaryotic homologues of the twinkle helicase suggests that it might retain a mitochondrial 
primase function, although the domains associated with this function are not well conserved in mammals ${ }^{19}$. While the functional identity of the primase enzyme remains elusive, the efforts to uncover the events following RNA priming have met with considerably more success. Given the coordination of transcription and replication of mtDNA, it is perhaps not surprising to find some of these same factors involved in the maintenance and organization of the mitochondrial genome. 


\section{Mitochondrial DNA Organization}

The mtDNA copy number in somatic cells is generally in the range of $10^{3}$ $10^{4}$ copies per cell, packaged in a DNA-protein structure called the nucleoid, at approximately 2-10 copies per nucleoid ${ }^{20-22}$. Gametes are a notable exception: mature oocytes have approximately 100,000 mtDNAs ${ }^{23}$ and sperm about $100^{24}$. Investigation of the protein constituents of the yeast mitochondrial nucleoid by mass spectrometry has revealed a large number of proteins, some of which have dual functions in nucleoid maintenance and tricarboxylic acid cycle activity ${ }^{25,26}$. The mitochondrial nucleoid in higher eukaryotes is reported to contain mTTFA, a mitochondrial transcription factor, single-stranded binding protein, twinkle (a helicase) and at least four additional inner membrane proteins ${ }^{27-29}$. Recently, a slew of new candidate proteins identified by cross-linking studies performed in mammalian mitochondria have been proposed ${ }^{29}$ but the authenticity of these candidates remains to be validated ${ }^{30}$. It is generally agreed that a few bona fide factors comprise the nucleoid and in vitro defined minimal replisome, including TFAM, TFB1, TFB2, POLG, SSB, TWINKLE. TFAM, originally named MtTFA, is a basic protein of the HMG box family that is thought to package mtDNA ${ }^{31}$. In vitro studies using Atomic Force Microscopy and Surface Plasmon Resonance have shown that TFAM protein is able to bind to mtDNA cooperatively and compact non-specific plasmid DNA in a protein-dependent fashion ${ }^{32}$, further solidifying its role in mtDNA maintenance. Moreover, decreasing mtTFA levels results in loss of mtDNA; likewise cells devoid of mtDNA contain no detectable mtTFA, leading to the suggestion that mtDNA levels are controlled by mtTFA ${ }^{33}$. 
MtDNA copy number varies widely from cell to cell, and is tightly regulated in a cell-specific fashion; however, replication of mtDNA is not tightly coupled to the cell cycle ${ }^{7}$. The first studies performed in cultured cells demonstrate that mitochondria located in a peri-nuclear position are the first to undergo mitochondrial DNA replication ${ }^{34}$, although other studies have not been successful in recapitulating this peri-nuclear bias. Thus, during mitosis some templates may replicate more than once, others not at all ${ }^{35}$. This behaviour, coupled with the random distribution of mtDNAs to daughter cells at cytokinesis provides a mechanism for the segregation of mtDNA sequence variants. It is important to point out that replicative segregation of mtDNA also occurs in post-mitotic cells, as mtDNA replication and turnover is an ongoing process throughout their lifetime ${ }^{36}$

\section{Mitochondrial DNA Transcription}

MtDNA is transcribed as three polycistronic units: the entire $\mathrm{H}$ and $\mathrm{L}$ strands and the two rRNAs, by a single subunit, phage-like RNA polymerase ${ }^{8}$. The rRNAs are transcribed 10-60 times more frequently than the entire $\mathrm{H}$-stand, a process controlled by a specific termination factor that binds to both the transcription initiation site, and a termination site located 3' to the 16S rRNA in the gene coding for tRNA ${ }^{\text {leu(UUR) } 37,38}$. The absence of coding information in the D-loop region was the first clue that drew researchers to investigate it as the control region for transcription initiation, but it was only with the advent of a system for in vitro transcription initiation in mitochondrial extracts that allowed for 
its molecular dissection ${ }^{39}$. Linker scanning mutagenesis of the D-loop region identified the essential promoter element sequences necessary for the initiation of transcription from the L- and $\mathrm{H}$ - strands (LSP and HSP). In mammals, approximately 150 base pairs separate these two, which are non-overlapping and independently functioning. The L-strand transcript is a polycistronic molecule that encodes 8 tRNAs and one polypeptide of complex I (ND6). Transcription from the $\mathrm{H}$-strand proceeds in the opposite direction under the control of two separate initiation sites, designated HSP1 and HSP2 ${ }^{40}$. The first of two overlapping transcripts code for tRNA ${ }^{\text {Phe }}$, tRNA ${ }^{\mathrm{Val}}$, and the large and small rRNAs: termination occurs at the 3' end of the small rRNA. The other polycistronic transcript is eventually processed, resulting in the other mRNAs and the remaining tRNAs. Mitochondrial transcription has been reconstituted in vitro and requires only the presence of two transcription factors, mtTFA and mtTFB (either B1 or B2), and the single subunit RNA polymerase (POLG) ${ }^{41,42}$. How the transcription machinery recognizes promoter sequences and initiates transcription is not fully understood, although it has been proposed that sequence-specific binding of TFAM upstream of the HSP and LSP alters the local conformation of mtDNA and allows for start site recognition. Maturation of the mitochondrial transcripts requires an RNAase $\mathrm{P}$ activity, and it is thought that the tRNA genes, which are interspersed between many of the protein coding genes, act as signal sequences in this process ${ }^{43}$. The regulation of transcription termination of the $\mathrm{H} 2$ transcript is mediated by mTERF1, which belongs to a family of recently-identified transcription factors (MTERF1-MTERF4) ${ }^{44}$. A 
termination site for the $\mathrm{H} 2$ transcript has been identified, although the identity of the transcription factors that bind this region upstream of the tRNA ${ }^{\text {Phe }}$ have yet to be revealed. While the actual functions of MTERF2 and MTERF4 have yet to be elucidated, studies in MTERF3 knockout mice indicate that it is a negative regulator of transcription ${ }^{45}$ while decreased synthesis of mitochondrial polypeptides observed in MTERF3 knock-down cells suggests a putative role in mitochondrial translation, perhaps bridging the mitochondrial transcription and translation apparatus ${ }^{46}$.

\section{Mitochondrial Translation}

Mitochondrial translation occurs on a dedicated apparatus, located in the mitochondrial matrix, that resembles that of prokaryotes ${ }^{47}$. Mitoribosomes are sensitive to antibiotics that inhibit prokaryotic translation, but they are remarkable in that they have a much higher protein/rRNA ratio ${ }^{48}$. In fact, almost half of the mitochondrial ribosomal proteins have no obvious orthologues with either prokaryotic ribosomal proteins or those in cytoplasmic ribosomes. The initiation, elongation and termination factors necessary for mammalian mitochondrial translation have all been cloned, but the regulation of translation remains poorly understood ${ }^{47}$. All of the proteins involved in the replication and expression of mtDNA are encoded in the nuclear genome and must be targeted to the mitochondria. 


\section{Part 2: Female Gametogenesis and Early Embryogenesis in the Mouse Origin and Development of the Germ Line}

The emergence and development of the female germ line in mammals is a complex and dynamic process involving the interaction the soma and the germ cells. Germline development begins with the specification of the primordial germ cells (PGCs), which are induced in the primitive ectoderm of the pre-gastrulating embryo. In the mouse embryo, primordial germ cells are first observed as a founding cluster of approximately 50 cells appearing posterior to the primitive streak between the endoderm and mesoderm of the ventral part of the amniotic fold. These large, round cells were first identified by the intense plasma membrane staining activity of tissue-nonspecific alkaline phosphatase (TNAP), which still remains the de facto histochemical marker for PGCs ${ }^{49,50}$. Beginning at embryonic day 6 (E6.0), which is 6 days post coitum (6 dpc), the earliest events of specification were investigated by single cell transcriptional profiling of nascent PGCs, leading to the identification of two genes, originally termed Fragilis and Stella ${ }^{51,52}$ that are believed to define PGC competence prior to germ cell fate determination. Further investigation of Fragilis (also referred to as IFITM3), revealed a cluster located on human chromosome 11 containing two other family members, IFITM1 and $2^{53}$. IFITM1 expression mediates the repulsive activity that drives the migration of PGCs from the mesoderm to endoderm, and while the role of IFITM3 is less clear, it seems that its activity is important in the regionalization of PGCs to the posterior endoderm. After E7.0, signaling from the overlying ectoderm by BMP4/BMP8 $b^{54,55}$ to the precursor population of nascent 
PGCs is absolutely necessary for further specification of germ cell fate. Mice lacking BMP4 function are unable to produce PGCs, while BMP8b null mice have a severely reduced number of germ cells.

Following PGC specification, these cells migrate from the posterior primitive streak whilst expressing a subset of well-characterized and spatiotemporally specific markers of the germ cell lineage. In addition to the IFITMs and Stella, Oct4, which belongs to the POU family of transcription factors, is required to maintain the pluripotency of the germ cell lineage. A transgenic mouse in which the PGC-specific promoter elements of Oct4 are used to drive expression of a GFP reporter has served as a useful tool to track the movements of PGCs in vivo ${ }^{56,57}$. At E8.5 the PGCs reside largely in and around the stalk of the allantois at the posterior end of the primitive streak. By E9.5, the PGCs have increased in number (approximately 200 TNAP-positive cells) when they exit dorsally and become embedded in the wall of the hindgut endoderm ${ }^{50}$. Molyneaux and colleagues have used time-lapse movies to show that the motility of PGCs is constant while migrating from the allantois, during which time cell division occurs every 16 hours ${ }^{58,59}$.

From E9 until the gonad colonization, the PGCs develop pseudopodia and other structures capable of amoeboid movement ${ }^{60}$, suggesting an active migration through the dorsal mesentery to the genital ridge. However, the proliferation and motility of these cells is not exclusively a cell-autonomous process. On the contrary, the somatic cells adjacent to the PGCs also play a role in maintaining the survival of PGCs as they migrate to their final destination. It 
has been suggested that the invaginating hindgut of the embryo facilitates PGC migration by sweeping the PGCs passively during gastrulation ${ }^{60,61}$. PGCs apparently do not to migrate independently, but rather form intercellular cytoplasmic processes, creating an extensive network ${ }^{61}$.

At E10.5, the gonads begin to form by proliferation of somatic cells at the genital ridge. PGCs begin to leave the hindgut endoderm and move along the dorsal mesentery toward the paired thickenings on the ventromedial surface of the mesonephros. At E11.5, the coalescence of germ and somatic cell leads to the formation of paired gonadal primordia. All the PGCs reach the gonad beginning around E12-13 in the mouse and E35 in humans ${ }^{62}$, at which time these cells are termed oogonia in females. In mice, the number of oogonia has been estimated at 4,000 to 11,000 per gonad. Sexual dimorphism becomes apparent morphologically as these cells proceed along sex-specific differentiation paths that are influenced by signaling events initiated by adjacent somatic cells of the mesonephros ${ }^{58,63}$. Concomitant with these events is the proliferation of oogonia until they become oocytes. These germ cells enter meiosis at around E13.5 in the mouse, when expression of markers such as TNAP and Oct-4 are downregulated. Concomitant with meiotic arrest at the diplotene stage, the oocyte surrounds itself with a layer of pregranulosa cells of somatic origin, creating a primordial follicle. Primary follicles arise once the proliferating granulosa cells assume a cuboidal shape and the zona pellucida synthesis begins. Oocyte growth rates to this point are rapid, with follicles increasing from $40 \mu \mathrm{m}$ (primary follicles) to $140 \mu \mathrm{m}$ preovulatory follicles found in humans. The 
establishment of the oocyte pools is a function of three parameters; i) the number of PGCs that reach the gonad ii) the mitotic activity of dividing oogonia iii) the attrition of oogonia during oogenesis. The dogma that female germline stem cells become extinct at birth has recently been challenged ${ }^{64}$, but the interpretation of the data in this latter study has been seriously questioned ${ }^{65}$.

Remarkably little is known about the metabolic activity or molecular biology of mitochondria during PGC specification and migration, or during oogenesis. Ultrastructural studies of human PGCs show that the number of mitochondria increases from about 10 in pre-migratory PGCs to about 200 in oogonia, and to several thousand by the diplotene stage of meiosis where they arrest ${ }^{66}$. There are no data on changes in mtDNA copy number during this period of development. There are approximately 4,500 cells in an E7 mouse embryo and 14,000 cells in an E7.5 embryo ${ }^{67}$. If the approximately 100,000 mitochondria in the zygote were simply apportioned to all cells in the early embryo, and there were no mitochondrial replication, PGCs (and somatic cells) would contain between 7 and 22 mitochondria, remarkably similar to what has been reported from transmission electron microscope (TEM) studies in humans ${ }^{68}$.

\section{Early Embryogenesis}

Following ovulation, the mammalian oocyte is released from the ovary fertilized while still in the oviduct. Fertilization causes the resumption of meiosis and the formation of the second polar body. Approximately 24 hours after fertilization, the first cleavage occurs, with subsequent cleavage divisions initiated 
at 12-hour intervals as the preimplantation embryo descends toward the uterus, progressing from the 2-cell to 4-cell stage. Completion of the third cleavage division in mice generates an 8-cell embryo, which undergoes a process known as compaction, where the blastomeres markedly increase the area of cell surface contact with each other by forming a solid ball of cells ${ }^{69,70}$.

The cells of the compacted embryo divide once again to generate a 16-cell morula composed of group a large of external cells surrounding by a group of smaller internal cells ${ }^{71}$. The external cells will give rise to the trophectoderm (Tr) while the internal cells will develop into the inner cell mass (ICM). The creation of two distinct groups of cells at this stage marks the first event of differentiation.

The ICM will give rise to the embryo proper as well as extraembryonic structures. For the most part, the larger group of cells, known as trophoblast cells, will not generate embryonic structures. Nevertheless, the trophoblast plays an integral role in the continued development of the mammalian embryo by initiating a process known as cavitation. At approximately 3.5 days' gestation (or $\mathrm{dpc}$ ), fluid is secreted into the morula by the trophoblast cells, creating an internal cavity known as a blastocoel (the embryo is henceforth referred to as a blastocyst). The blastocyst expands considerably within the confines of the zona pellucida, which acts as a barrier preventing the premature adhesion of the embryo to the wall of the oviduct as it descends to the uterus.

Between 3.5 and 4.5 days' gestation (or dpc), the ICM becomes divided into two regions. The surface layer in contact with the blastocoel will develop into the primitive endoderm, contributing to the extraembryonic membranes such as 
the yolk sac, allantois, and amnion. The remaining cells of the ICM will develop into primitive ectoderm or epiblast. By 4.5 days after fertilization, the expanding blastocyst is extruded after lysing a hole in the zona pellucida. Once hatching is completed, the blastocyst is ready to make contact with the uterine epithelium and initiate a cascade of enzymatic events that will allow the embryo to bury itself within the uterine wall ${ }^{72,73}$.

At implantation, the cells of the mural trophectoderm at the region not in apposition with the ICM undergo DNA replication in the absence of cytokinesis. This generates the so-called trophoblast giant cells, which are essential for the successful invasion of the embryo into the uterine epithelium. The remaining trophectoderm cells develop into the ectoplacental cone and the extraembryonic ectoderm, both of which contribute to the development of the placenta.

By 6 days post-fertilization, an internal cavity within the epiblast has begun to form. The epiblast, now wrapped around the proamnitoic cavity, is a thin layer of epithelium resembling a cup and consisting of about 1000 cells that will eventually develop into the embryo proper. Mitochondrial DNA replication is thought to resume in the cells of the embryo at this time. By E6.5, the anteriorposterior axis of the embryo first becomes visible and the first events of gastrulation are executed, which have been suggested to play a role in the migration of the emerging primordial germ cells. 


\section{Part 3: Germline Transmission of Mitochondrial DNA}

\section{Segregation of Mitochondrial DNA in the Female Germline}

Most mammals have a single mtDNA sequence variant in all of their cells, a condition referred to as mtDNA homoplasmy. The relative rarity of mtDNA heteroplasmy (the occurrence of more than one sequence variant in an individual) and the high degree of population polymorphism suggest that new mtDNA sequence variants are rapidly segregated in maternal lineages. This seemed paradoxical given the high mtDNA copy number $\left(\sim 10^{5}\right)$ in mature oocytes, and the relatively small number of cell divisions in the development of the female germline, and suggested the existence of a genetic bottleneck for the transmission of mitochondria and mtDNA (Figure 2).

The concept of a bottleneck was first proposed by Hauswirth and Laipis ${ }^{3}$ to explain the rapid segregation of a mtDNA D-loop sequence variant in several maternal lineages of Holstein cows. Complete switching of the same allelic variant was observed in a single generation in $40 \%$ of the mother-daughter pairs examined in another study ${ }^{74}$. Segregation of many different pathogenic mtDNA sequence variants has also been observed in a large number of human pedigrees, however, it is rarely as rapid as that observed in the D-loop of cows $^{4,75}$. The mtDNA copy number is not known in either PGCs or in oogonia; 
Figure 2

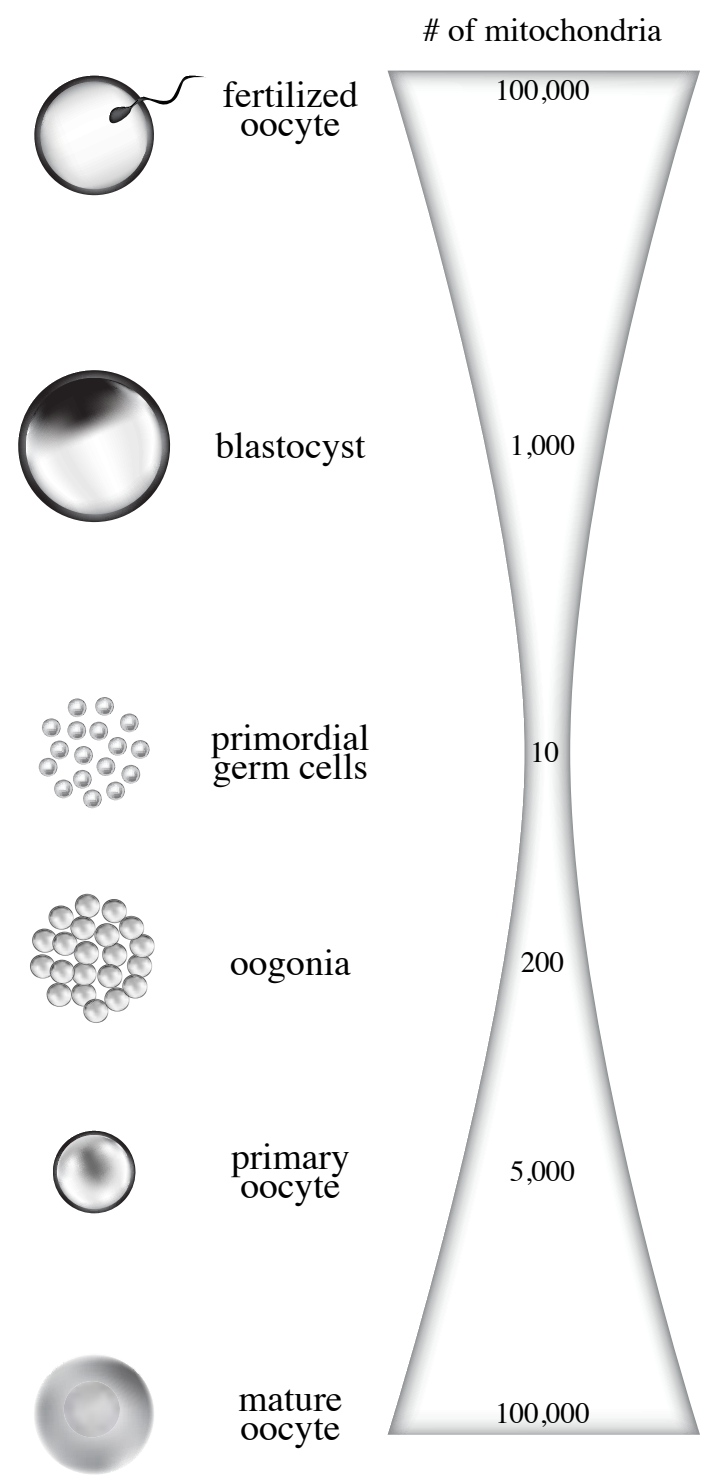


however, it has been speculated that mtDNA copy number may be 1 copy/organelle at all stages of gametogenesis ${ }^{66}$. The ultrastructural evidence clearly demonstrates a physical bottleneck in the number of mitochondria in the PGC population; however it is possible that mtDNA replication is not coupled to mitochondrial biogenesis prior to specification of the PGC population such that each mitochondrion in the PGCs contains multiple mtDNAs. Replication of mtDNA does not appear to restart until after the blastocyst stage ${ }^{23,76}$, although one study suggests that mtDNA replication occurs during a very narrow window (1-2 cell stage) of development in the preimplantation embryo, albeit without a change in mtDNA copy number ${ }^{77}$. Assuming that replication of mitochondrial DNA is halted until postimplantation, and it is worthwhile to note that no direct measurements of mitochondrial DNA replication have been performed, it is therefore likely that the number of copies of mtDNA in PGCs lies somewhere between 10 and 100 . The 10,000-fold reduction in organelle number from the zygote to the PGCs may simply be the result of a failure to restart zygotic replication of mitochondria, but whether that is the result of the absence of some key factor, or whether mitochondrial biogenesis is actively repressed remains unknown.

Studies of the transmission of polymorphic mtDNA sequence variants in heteroplasmic mice using single cell PCR on primordial germ cells and oocytes ${ }^{4}$ have shown that segregation is essentially complete by the time the primary oocyte population is differentiated. Surprisingly, little intercellular variation in the degree of mtDNA heteroplasmy was found amongst individual PGCs sampled 
from individual embryos (although it must be said that the number of PGCs analyzed was quite small). These data imply that the PGCs contain a more or less representative sample of the mitochondria in the zygote, and that the segregation of mtDNA sequence variants occurs by replicative segregation during the expansion of the oogonial population. The observation that the mean level of heteroplasmy in a large sample of offspring from single mothers was not significantly different than the level of heteroplasmy in the mother suggests that mtDNA segregation between generations occurs principally by random genetic drift ${ }^{4,78}$. Studies of homopolymeric tract heteroplasmy in humans are consistent with the mouse studies, showing that segregation has occurred by the time oocytes are mature ${ }^{78}$.

An attempt was made to estimate the effective number of segregating units of mtDNA in the germline using a population genetic model that takes into account the number of mitotic divisions in the development of the germline $(\sim 15)$, but assumes a constant mtDNA copy number during gametogenesis ${ }^{4}$. While the latter is clearly not the case (mtDNA copy number steadily increases during germline development), the analysis at least provides a basis for comparison, though it will tend to overestimate the number of segregating units, as the rate of mtDNA segregation is inversely related to copy number. Using this model, the number of segregating units of mtDNA was estimated at 200 in the mouse ${ }^{4}$, in broad agreement with estimates obtained from human pedigrees segregating pathogenic point mutations, and nearly identical to that obtained by direct evaluation of heteroplasmy in the oocytes from a patient carrying a pathogenic 
tRNA mutation ${ }^{79}$. However, it does not seem that all mutations are treated equally in the germ line. Two studies have investigated oocytes or early embryos from mothers carrying the T8993C NARP (Neuropathy, Ataxia, and Retinitis Pigmentosa) mutation in the ATP6 gene, and have shown almost complete segregation of this mutation, suggesting that the bottleneck for transmission of this mutation might be extraordinarily narrow ${ }^{80,81}$. Consistent with extreme skewing of heteroplasmy in the germ line in these cases, a disproportionate number of reports of apparently de novo mutations in the ATP6 gene have appeared $^{82}$.

The pattern of transmission of mtDNAs has also been analyzed in a large number of pedigrees segregating six of the most common pathogenic mtDNA point mutations (see Figure 1): A8344G (MERRF, Mitochondrial Encephalomyopathy, Ragged-Red Fibres), A3243G (MELAS, Mitochondrial Encephalomyopathy, Lactic Acidosis, Stroke-like Episodes), T8993C (NARP, Neuropathy, Ataxia, Retinitis Pigmentosa), T8993G (NARP), G11778A (LHON, Leber's Hereditary Optic Neuropathy), G3460A (LHON) ${ }^{83}$. If the transmission of pathogenic mtDNAs were stochastic, then the proportions of mutant mtDNAs in the offspring of carrier mothers should be symmetrically distributed about the value in the transmitting female; in other words the proportion of mutant mtDNAs would be just as likely to increase as decrease. Transmission of the pathogenic mutation was not different from that predicted by a stochastic model for two of the mutations, and for three of the mutations there was a significant increase in 
mutant mtDNAs in the offspring. Only one showed a very small shift toward the wild-type sequence.

The creation of animal models segregating specific pathogenic point mutations in mtDNA has been plagued with difficulties, due in large part to the inability to effectively transform mammalian mitochondria. This problem has been circumvented in one instance by transferring naturally occurring large-scale mtDNA deletions to one-cell embryos using enucleated cytoplasts as the transfer vehicle ${ }^{84}$. In humans these large deletions are usually associated with progressive external ophthalmoplegia (PEO) or Kearns-Sayre Syndrome (KSS), both of which are nearly always sporadic diseases ${ }^{85}$. The mice were able to transmit the deleted species of mtDNA at high levels (greater than $80 \%$ mutant mtDNAs in some animals) through three generations, clearly showing that there is no barrier to the transmission of pathogenic mtDNA mutations in this model, and no loss of oocytes with high proportions of mtDNA deletions. By contrast, the risk of transmitting a large-scale mtDNA deletion in humans is only about $4 \%{ }^{81,86}$. It is not known why the human and mouse differ in their ability to transmit these particular mtDNA mutations, but it likely reflects the fact that humans with high proportions of large-scale mtDNA deletions have a severe clinical phenotype and rarely reproduce.

The above data, while still limited, suggest that the transmission of polymorphic and pathogenic mtDNA sequence variants in mammals is primarily a stochastic process, and that the presence of a genetic bottleneck in the PGCs results in 
more rapid sorting out of new mutations than expected based on the high genome copy number in the zygote.

\section{Oxidative phosphorylation, atresia and oocyte quality}

Most of the oocytes that are formed during fetal life die by atresia. About half die before birth and the process of wastage is continuous throughout life. Thus, in humans, of the roughly $10^{6}$ primary oocytes present at birth, about $3 \times 10^{5}$ remain at sexual maturity, and $10^{3}$ at menopause ${ }^{87}$. An evolutionary argument has been advanced suggesting that mitochondrial function might be the basis for atresia ${ }^{88}$. The argument is based on the strong correlation between the size of the genetic bottleneck for mtDNA and the fraction of atretic cells determined in a number of different animal species. Mitochondrial function has also been suggested to be important contributor to oopause and to oocyte ageing ${ }^{66,68}$, and the introduction of mitochondria into rodent oocytes can reduce the number undergoing apoptosis in vitro ${ }^{87}$. However, several lines of evidence argue against the idea that any of these effects are based on the capacity for energy production by oxidative phosphorylation in the oocyte.

First, mtDNA diseases are a significant medical problem. Full-term, apparently normal babies, with very high proportions of pathogenic mtDNA mutants, are born to heteroplasmic carrier mothers. These babies only become symptomatic after birth when there is increased demand for oxidative phosphorylation and an upregulation of mitochondrial biogenesis. The simplest interpretation of these facts is that stringent selection for oxidative 
phosphorylation function does not exist, either during oogenesis, or at any stage of fetal life. If atresia were a filter for oxidative phosphorylation capacity, it would effectively prevent the transmission of oocytes with mtDNA mutations, because the mutations would trigger a suicide response at some stage of oogenesis. Second, it is very difficult to imagine how new germline mutations could be selected against at any stage of oogenesis, because they would initially be rare. The expression of a pathogenic phenotype due to an mtDNA mutation usually depends on reaching a critical threshold of mutant mtDNAs before a biochemical or clinical abnormality is observable. These thresholds can range from $30-85 \%$ of mutant mtDNAs ${ }^{89,90}$, so it is unlikely that a small proportion of mutant mtDNAs could be selected against. Even if there were a mechanism to select against such mutants, the number of oocytes harbouring such mutations would be orders of magnitude less than the numbers of oocytes that die by atresia. Finally, atresia that occurs late in development is initiated by the somatic granulosa cells ${ }^{87}$, and it is difficult to see how the capacity for oxidative phosphorylation in the oocyte would initiate an apoptotic cascade in the surrounding follicular cells.

What about mitochondrial function and oocyte ageing? A number of studies have investigated the occurrence of mutations in ageing oocytes, usually using as a marker the presence of the so-called common deletion associated with Kearns Sayre Syndrome ${ }^{91}$. Although these studies have generally been able to detect this mutation in some fraction of oocytes, the proportion of mtDNAs affected has always been very small, and there is not a reproducible correlation with maternal age ${ }^{92-96}$. It is probably premature to completely discard the notion 
that some aspect of mitochondrial function is important in oocyte quality, but there is currently no compelling evidence to suggest that this correlates with the capacity for oxidative phosphorylation.

\section{MtDNA copy number in the Oocyte}

The idea that mitochondrial function is an important determinant of oocyte quality has lead to a recent barrage of studies trying to demonstrate that oocyte mtDNA content, and by inference oxidative phosphorylation capacity during early embryogenesis, is a marker for female infertility. In this light, several groups have suggested that oocytes from women with premature ovarian failure or from older donors have a reduced ability to generate viable embryos, owing to a reduced mtDNA copy number. This argument could have some merit, not because of reduced copy number impairs oxidative phosphorylation capacity in the oocyte in an important way, but rather because reduced mtDNA copy number (and the associated reduced mitochondrial numbers) in the zygote would be predicted to affect the distribution of mitochondria in the cells of the early embryo at a period when mitochondrial biogenesis is slowed or arrested. These studies have generated several estimates of mtDNA copy number in presumably healthy

metaphase II human oocytes ranging from 50,000 to $1,500,000$ copies $^{96-100}$. The range in the estimates of mtDNA copy number ( 30 -fold) is at least 10 -fold greater than earlier measurements made by Southern Blot in murine and bovine oocytes 23,101. 
There are a number of possible explanations for the variability in the estimates of mtDNA copy number in human oocytes as compared to those in other mammals. First, it may simply reflect the fact that the estimates from mice and cows are derived from animals with a more homogeneous nuclear genetic background than the human population. Second, the observed variability may reflect real biological variation in the sample of oocytes used in these studies. For obvious ethical reasons, it is seldom possible to harvest oocytes from normal healthy women for research purposes. Invariably, researchers must turn to IVF clinics to recover ooctyes from women who have given informed consent to donate their surplus oocytes. As a result, the oocyte quality and donor fertility are likely subject to stratification. Thus it seems unlikely that oocytes harvested from this cohort accurately reflect random sampling from a healthy population. A third and more disconcerting cause of the inter- and intra-study variation in mtDNA copy number variability may have to do with the use of the Quantitative Real-Time PCR platform that all the recent studies have used to estimate copy number. Real-Time PCR is a method of simultaneous DNA amplification and quantification. After each round of amplification, the DNA is quantified by measuring a fluorescent signal that is generated immediately following the synthesis of DNA, as it intercalates with a fluorescent dye such as SYBR Green. Tracking the fluorescence as the reaction progresses, it is possible to monitor the early cycles of the log-linear phase of amplification. In theory, there exists an inverse relationship between the quantity of starting material and the fluorescence generated during the log-linear phase of amplification. This is 
reported to the user as a Ct value, which represents the PCR cycle number at which the fluorescence is detectable above a predefined threshold. Using a standard curve generated from serial dilutions of a PCR product or plasmid of known concentration, Real-Time PCR machines can automatically extrapolate unknown starting concentrations from Ct values and provide the user with an absolute starting template number. Under the appropriate conditions, Real-Time PCR quantification of nucleic acid can be a powerful and sensitive platform, rivaling all other detection methods including hybridization techniques (i.e. Southern Blots, Northern Blots) and traditional end-point PCR analyses.

\section{Male Transmission of mtDNA}

There are only rare exceptions to the general rule of uniparental inheritance of organellar genomes, either mitochondrial or chloroplast ${ }^{102}$, and it has been suggested that this nearly universal phenomenon may have evolved to

limit the spread of selfish genetic elements ${ }^{103}$. The lack of a paternal contribution of mitochondrial DNA has been attributed to a variety of factors, including dilution and active elimination. That the mitochondrial DNA content of the mammalian male gamete is estimated to be 3 orders of magnitude less than its female counterpart has caused some to invoke a simple dilution of paternal mtDNA in the oocyte at fertilization as the mechanism that makes transmission to the next generation unlikely. While this may be the most parsimonious explanation for the paucity of paternal mtDNA transmission in mammals, others have invoked a mechanism of active exclusion whereby active destruction of paternal mitochondria (which are 
present in the sperm midpiece that penetrates the oocyte during fertilization) in the zygote lead to uniparental inheritance of mitochondria and their genomes ${ }^{104}$. The surveillance system that targets paternal mtDNA can apparently be bypassed in interspecific crosses, as shown in studies with mice ${ }^{105,106}$. Gyllensten and colleagues discovered that successive backcrossing between species of mice for up to 26 generations could yield animals harbouring low levels of paternally derived mtDNA in all of their tissues. In a similar study using the same interspecific crosses between M.m. domesticus $x$ M. spretus, paternal mtDNA was detected by PCR analysis in early embryos and in about half of all offspring generated from these crosses ${ }^{107}$. Unlike in the earlier report, the distribution of paternal mtDNA in tissues obtained from these positive offspring was non-uniform. Fewer than $10 \%$ of hybrid females generated from this interspecific cross possessed paternal mtDNA in their ovaries, and none of the unfertilized oocytes from these animals were positive for the presence of paternal mtDNA. These observations lead to the notion that sperm from closely related species or sub-species may perchance escape the normal surveillance mechanisms put in place to ensure strict matrilineal transmission of mtDNA. Interestingly, no paternal mtDNA was detected in any of the $\mathrm{N}_{2}$ backcross animals (tested by examining embryos obtained by in vitro fertilization), suggesting some barrier to transmission even in $F_{1}$ hybrid animals, and that the genetic factors responsible act as either a dominant or co-dominant maternal trait. Other mammalian examples of paternal transmission (also referred to as paternal leakage) of mtDNA have been reported in sheep ${ }^{108}$ using similar PCR- 
based methods. For instance, Zhao and colleagues demonstrated the occurrence of leakage in two related hybrid families (out of a total of 48) produced by Dorset crossed to Small Tail Han sheep. Taken together, these results suggest that the molecular targets for paternal mtDNA elimination in intraspecific crosses is a nuclear-encoded factor(s) associated with the sperm midpiece.

It has been widely accepted that spermatozoa are translationally silent, but a recent study has challenged this dogma ${ }^{109}$. Gur and Breitbart showed that labelled amino acid incorporation was completely blocked by mitochondrial translation inhibitors, but not by a cytoplasmic 80 s ribosomal inhibitor. This suggests that mammalian sperm translates nuclear-encoded proteins by mitochondrial-type ribosomes that are important in the fertilization of the oocyte. Sutovsky and colleagues have suggested that mitochondria are earmarked for destruction by the addition of ubiquitin tags ${ }^{104,110}$. The ubiquitination of sperm occurs in the male reproductive tract at the secondary spermatocyte/round spermatid stage, is masked during epididymal passage (possibly by disulphide bond cross-linking), and reappears after fertilization ${ }^{111}$. Although markers of sperm mitochondria and anti-ubiquitin antibodies co-localize by immunofluorescence in early embryos, there is no direct evidence that the mitochondria themselves are tagged. The destruction of sperm mitochondria could, however, be prevented by the injection of anti-ubiquitin antibodies, or an antagonist of proteosome activity ${ }^{111}$, suggesting that ubiquitination is an important signal in the degradation pathway of paternal mitochondria. However, it is unclear how the nuclear gene products required for ubiquitin-mediated protein 
degradation can be differentially recognized in closely related species by the oocyte proteosome.

More recently, it was shown that the paternal mitochondrial genome in fish is actively removed prior to the purported degradation of sperm mitochondria. Using optical tweezers to remove intact sperm from within freshly fertilized fish eggs, Nishimura and colleagues showed that paternal mtDNA was in fact actively degraded well before the disappearance of the paternal mitochondria ${ }^{112}$. While the elimination of paternal organellar DNA seems to be a conserved mechanism used to ensure matrilineal transmission, the nature of such a nucleolytic mechanism remains a mystery. Currently, there are no data on how paternal mtDNA is actively eliminated from the mammalian oocyte.

Recently a single case of paternal transmission of a pathogenic mtDNA mutation was reported in an individual with a severe muscle myopathy ${ }^{113}$. This case prompted other investigators to search for paternal transmission retrospectively in a large group of patients ${ }^{114,115}$. However, no evidence of paternal transmission was found in these studies, suggesting that it is likely to be an extremely rare event in human biology. 


\section{Thesis Outline:}

Most eukaryotic cells rely on adenosine triphosphate (ATP) produced by oxidative phosphorylation (OXPHOS) for their normal function. This process requires the activity of five multi-subunit enzyme complexes located in the inner mitochondrial membrane. These multimeric complexes are unique in the cell in that the component polypeptide subunits are encoded by both the nuclear and mitochondrial (mtDNA) genomes. Of the approximately 80 structural subunits of oxidative phosphorylation, 13 are mtDNA-encoded, and all are essential for function. MtDNA is maternally inherited in mammals, and as mitochondria are not made de novo, but rather elaborated from other mitochondria, all of our mitochondria and mtDNAs ultimately derive from those in one of our mother's oocytes.

Mutations in mtDNA are an important cause of a group of multisystem disorders usually referred to as mitochondrial encephalomyopathies, because of the prominent involvement of the nervous system and striated muscle ${ }^{85,116}$. The minimum prevalence of these disorders has been estimated at about 1:5000,

making them one of the most common inherited metabolic diseases ${ }^{83,116}$. In most patients, pathogenic mtDNAs co-exist with wild-type mtDNAs (a state referred to as heteroplasmy), and a variable proportion of mutant mtDNAs can be transmitted to the next generation. The proportion of mutant mtDNAs transmitted from mother to child is the most important determinant of the onset and severity of disease. The rules governing transmission and segregation of mtDNA in the female germline have thus taken on a renewed importance for genetic counseling 
and the clinical management of patients affected by these disorders. In addition, the introduction of new reproductive technologies (such as cytoplasmic transfer ${ }^{117-119}$ ), have forced a re-evaluation of the potential contribution of exogenous mitochondria to the next generation.

Mammalian oocytes contain at least 100,000 mitochondria and mtDNAs, yet sequence variants segregate rapidly between generations, due to the presence of a bottleneck in development ${ }^{3,4}$. The size and shape of this bottleneck are not known, nor is it known how decreases in mtDNA copy number or diminished OXPHOS function might affect mtDNA transmission. It is widely assumed that the large number of mitochondria in the oocyte are required for energy production, and that diminished mitochondrial function contributes to germ cell atresia ${ }^{66,88}$, and the deterioration in oocyte quality with age ${ }^{66,98,120}$; however, these concepts are not easy to reconcile with the fact that pathogenic mtDNA mutations are transmitted, without evidence of reduced fertility. An alternate hypothesis is that the large number of mitochondria in the ooctye exists simply as a genetic mechanism to ensure that all cells in the early embryo receive mitochondria containing mtDNA. In this view aerobic energy production is of relatively little importance in oogenesis, but mtDNA copy number has a major influence on germline competence. Nevertheless, the physiological threshold of mtDNA copy number (in the oocyte and sperm) required for proper fertilization and embryogenesis remains undefined.

Chapter 1 of this thesis will address the rapid segregation of mitochondrial DNA in the female germline. I will demonstrate that the segregation patterns 
observed in the offspring of wild type heteroplasmic females segregating BALB and NZB mtDNA (Table 1) is the outcome of a mitochondrial genetic bottleneck, caused by the replication of a subgroup of genomes in the early postnatal ovary. I will then propose a model for the transmission of mtDNA. Chapter 2 will seek to validate this model by examining the effects of altered mtDNA copy number and OXPHOS function in the developing germline on the distribution of heteroplasmy in offspring and germ cells. Finally, in Chapter 3 I will evaluate the impact mtDNA copy number on the outcome of gametogenesis in order to define a crucial developmental threshold of mtDNA. 


\title{
Chapter 1
}

The mitochondrial DNA genetic bottleneck results from replication of a subpopulation of genomes during oocyte maturation in early postnatal life

\author{
Timothy Wai, Danielle Teoli, and Eric A. Shoubridge
}

Originally published in Nature Genetics 2008 Dec;40(12):1484-8.

Permission to reproduce this manuscript has been granted by Nature Publishing

Group according to their copyright regulations 


\begin{abstract}
In mammals, mitochondrial DNA (mtDNA) sequence variants are observed to segregate rapidly between generations despite the high mtDNA copy number in the oocyte. This has led to the concept of a genetic bottleneck for the transmission of mtDNA ${ }^{3,121,122}$ but the mechanism remains contentious. Several studies have suggested that the bottleneck occurs during embryonic development, the result of a marked reduction in germline mtDNA copy number 4,5. Mitotic segregation of mtDNAs during preimplantation ${ }^{5}$, or during the expansion of primordial germ cells (PGCs) before they colonize the gonad ${ }^{4,5}$ is thought to account for the increase in genotypic variance observed among mature oocytes from heteroplasmic mothers. This view has, however, been challenged by studies suggesting that the bottleneck occurs without a reduction in germline mtDNA content ${ }^{6}$. To resolve this controversy we measured mtDNA heteroplasmy and copy number in single germ cells isolated from heteroplasmic mice. By directly tracking the evolution of mtDNA genotypic variance during oogenesis we show that the genetic bottleneck occurs during postnatal folliculogenesis, and not during embryonic oogenesis.
\end{abstract}




\section{Results and Discussion}

Hauswirth and Laipis proposed two hypotheses to explain the rapid segregation of neutral mtDNA variants between generations, first observed in Holstein cows: amplification of a small pool of mtDNAs at some point during oogenesis ${ }^{3}$ or unequal partitioning of mtDNA in the early embryo ${ }^{122}$. We previously constructed a heteroplasmic mouse model carrying functionally neutral BALB and NZB mtDNA sequence variants to test these hypotheses ${ }^{4}$. As the genotypic variance measured in the PGCs of these mice was markedly less than the genotypic variance in postnatal germ cells or live offspring, we concluded that random genetic drift due to mitotic segregation of mtDNAs was likely sufficient to account for the increase in genotypic variance in mature oocytes. This hypothesis was apparently corroborated by morphological observations revealing large differences $\left(10^{3}-10^{4}\right)$ in mitochondrial number between PGCs and oocytes in humans ${ }^{68}$.

Using quantitative PCR, Cao et al. ${ }^{6}$ estimated mtDNA copy number in embryonic germ cells (identified by TNAP activity) in E7.5-E13.5 mouse embryos and failed to find any significant changes in mtDNA levels during embryonic germline development. They estimated mean mtDNA copy numbers of $1,500-$ 3,000 and 100 mitochondria per cell. Thus, each mitochondrion was suggested to contain 15-30 mtDNAs, far more than had previously been reported in mammalian oocytes ${ }^{101}$ or any mammalian cell. Without a change in germline mtDNA levels it would be virtually impossible for rapid segregation of mtDNA to occur during the expansion of the PGC population, and so they were forced to 
conclude that the genetic bottleneck was due to a subgroup of mtDNAs undergoing preferential replication during oogenesis. In sharp contrast, using a Stella-GFP mouse to isolate individual PGCs, Cree et al. ${ }^{5}$ estimated $\sim 200$ copies of mtDNA in PGCs at E7.5 and $\sim 1,300$ copies at E14.5. Using a mathematical simulation they predicted that $70 \%$ of the mtDNA genotypic variance in the offspring of heteroplasmic mothers was generated by unequal partitioning of mtDNA in the pre- and early postimplantation embryo, the remainder being generated during the mitotic expansion of the PGCs.

We reasoned that a definitive test of the role of germline mtDNA copy number in the genetic bottleneck would require direct measurements of both genotypic variance and mtDNA copy number during the development of the female germline, and a method to test for statistically significant changes in genotypic variance at each stage of oogenesis. We first isolated embryonic germ cells from heteroplasmic mice expressing EGFP from a modified OCT4 promoter ${ }^{123}$ and made single-cell measurements of mtDNA copy number and heteroplasmy. The reporter transgene eliminates the need for histochemical stains, which had previously been used to isolate the cells of the germline ${ }^{49}$, but which confound mtDNA measurements made by quantitative PCR ${ }^{5,6}$ (Shoubridge \& Wai, data not shown). In manually isolated EGPF-positive germ cells, we detected significant changes in mitochondrial DNA copy number during early oogenesis between E8.5 (mean 280 copies, median 145), the earliest day at which we could unambiguously isolate these cells in the mouse strain we used, and E10.5 ( mean 2,800 copies, median 2200) (Fig. 1, top panel), similar to 
measurements made in PGCs isolated from the Stella-GFP mouse model ${ }^{5}$. Stella is one of the earliest markers of PGC specification, which occurs between E6.25 and E6.5 in the mouse ${ }^{124}$. E6.5 embryos contain about 910 cells $^{67}$, so if mtDNA replication were completely arrested until this stage of development, individual cells would be expected to contain 192 copies of mtDNA. Thus it is likely that the resumption of mtDNA replication in early development is coincident with PGC differentiation. By E14.5, when the PGCs have colonized the gonad, there are about 6,000 copies per cell. We observed significantly less mtDNA copy number variability in early PGCs than reported by Cree et al. ${ }^{5}$, (standard deviation 377 vs 699), a fact that we attribute to the manner in which the cells were collected. Manual isolation assures the exclusion of debris and doublets, which in our hands, occurred at an unacceptable frequency, even under the most stringent FACS gating parameters (data not shown). We conclude that there is in fact a severe decrease in mtDNA copy number in PGCs, at least 700 -fold relative to the mtDNA copy number in fertilized oocytes, and that it increases $10-20$ fold during the expansion of the PGC population prior to colonization of the gonad.

Strikingly, the large increase in mtDNA copy number during embryonic germline development does not lead to a concomitant increase in genotypic variance at any point during embryonic oogenesis when tested by a nonparametric test of equal variance (see Methods) (Supplementary Fig. 1). It is important to note that our analyses of genotypic variance make no a priori assumptions regarding the organization of the mitochondrial nucleoid, the nature or number of segregating units, or the number of germline mitotic divisions 
required for the genesis of mature oocytes. Rather, these analyses simply allow us to detect increases in genotypic variance, which is a direct measure of the segregation of mtDNA sequence variants. In this regard, our results directly contradict those of Cree et al. ${ }^{5}$, who did not examine levels of heteroplasmy directly in germ cells, but rather based their prediction of the evolution of heteroplasmy variance in early embryological development on a mathematical simulation. Actual measurements of genotypic variance in early PGCs in this study and in our previous study ${ }^{4}$ are inconsistent with significant mtDNA segregation before implantation, and even when we do all possible pair-wise comparisons at different stages of embryonic oogenesis, we fail to find significant differences in genotypic variance (Supplementary Fig. 1). We conclude that despite the severe reduction in mtDNA copy number, the mitochondrial genetic bottleneck does not occur during embryonic oogenesis.

We extended our analyses postnatally and discovered an inequality of genotypic variance (Supplementary Table 1) between all groups of germ cells containing more than 10,000 copies of mtDNA/cell (mature ovulated oocytes, primary oocytes in secondary follicles) and those groups of germ cells containing fewer than 10,000 copies of mtDNA/cell (PGCs, oogonia, primary oocytes in primordial follicles) (Fig. 1, Supplementary Fig. 1). These results indicate that the genetic bottleneck must be the result of the selective replication of a random subset of mtDNA templates during the growth and maturation of the ovarian follicles. This likely starts in the primordial follicles, as this subpopulation of 
mtDNAs would have to expand significantly in order for us to detect a change in genotypic variance in the oocytes derived from secondary follicles.

To confirm these genetic observations, we injected early postnatal mice (P1 to $\mathrm{P} 4)$ with the thymidine analogue BrdU to label replicating mtDNA. Following short (2 hr) and long BrdU pulses (24 hr), we harvested tissues for sectioning and immunohistochemical analyses. mtDNAs labeled with BrdU represent a small fraction of the total cellular nucleoid content in oocytes in both primordial and primary follicles as visualized with antibodies against either TFAM (Fig. 2), mt-SSB (Supplementary Fig. 2), or POLG (Supplementary Fig. 3), markers of the mammalian nucleoid ${ }^{125}$, confirming the predictions of the genetic analysis. TFAM-positive punctae exhibit a reduced degree of co-localization to BrdU-positive punctae in ovarian sections (Fig. 2 A, B), as compared to other tissues such as the heart (Fig. 2C, D), and particularly when compared to cultured cells (Supplementary Fig. 2), an observation that was recapitulated using POLG and mt-SSB antibodies (Supplementary Fig. 1). Although it is not clear why established markers of the mitochondrial nucleoid such as TFAM variably colocalize with foci of BrdU incorporation, there could be a number of explanations. We have previously shown that TFAM binds DNA non-specifically with nanomolar affinity and can completely coat and compact plasmid DNA molecules ${ }^{32}$. It is likely that this tight association needs to be modulated in order for the replication machinery to gain access to the DNA template, leading to reduced TFAM content on replicating mtDNAs, or to a change in epitope accessibility. It is worth noting that while BrdU-positive, TFAM-negative foci have been previously observed by 
immunocytochemistry in transformed cultured cells ${ }^{21}$ this observation has generally been ignored. It is entirely possible that this population represents actively replicating templates, which subsequently acquire additional TFAM and become compacted when replication terminates. Thus the relative proportion of nucleoids that stain positive for both TFAM and BrdU might simply reflect the rate of mtDNA replication in a particular cell or tissue.

Immuno-EM in the same postnatal ovaries demonstrates that both antiTFAM and anti-BrdU immunoreactivity were confined to the mitochondrial compartment (Fig. 4C, D). Using either antibody, we rarely observed more than a single focus of immunoreactivity (which we interpret as a nucleoid) in an individual mitochondrion (range 0-2) consistent with the idea that mitochondria in oocytes contain a small number of mtDNAs.

Coincident with the mitochondrial genetic bottleneck is the appearance of the Balbiani body $(\mathrm{Bb})$, which contains Golgi elements surrounded by mitochondria and endoplasmic reticulum ${ }^{126,127}$. This structure is evolutionarily conserved and has been suggested to play a role in mitochondrial inheritance ${ }^{128}$, so we asked whether the Balbiani body might influence the selection templates for replication. We observed a heterogeneous distribution of TFAM-positive mtDNA nucleoids located primarily about the GM130-positive Bb in primary oocytes (Figure $3 \mathrm{~A}$, Figure 4A,B). Following maturation through to the secondary follicle stage, the distribution of mitochondria and mtDNA nucleoids became more homogeneous and the $\mathrm{Bb}$ dispersed (Figure 3B). However, immunohistochemical analyses at the EM or confocal microscope level 
Supplementary Fig. 4) showed that replicating mtDNAs did not exclusively colocalize with the $\mathrm{Bb}$ (Fig. 4). Thus the $\mathrm{Bb}$ does not have an obvious role in the segregation of mammalian mitochondrial DNA. It is, however, possible that mitochondria are moving in and out of the $\mathrm{Bb}$, so we cannot entirely exclude a role for this structure in the selection of mtDNA templates for replication.

If the genetic bottleneck does not occur during embryonic oogenesis, what purpose is served by the severe reduction in mtDNA copy number that occurs in the earliest PGCs? Recent studies ${ }^{129,130}$ have suggested that mtDNA undergoes purifying selection in the germline, eliminating severe mutations. Successive backcrosses of a mutant line carrying a severe frameshift mutation in ND6, led to the rapid elimination of the mutant genome within 3 generations ${ }^{129}$, demonstrating that this filter can extinguish deleterious mutations well below the mutant loads that would be predicted to cause a biochemical deficit at the cellular level. If the mitochondrial genetic bottleneck were responsible for this selective elimination, one would predict that the genotypic variance observed in mature oocytes carrying such mutations would be reduced. To test this, we compared the genotypic variance reported in 12 oocytes from the mouse segregating a deleterious ND6 frameshift mutation ${ }^{129}$ with that which we had previously measured in ovulated oocytes, embryos, or live offspring from our heteroplasmic mice (data not shown), and found no statistically significant differences. As purifying selection decreases genetic variance, this comparison suggests that the mechanism responsible for the selective elimination of severe mutations acts before the genetic bottleneck determined in our mouse model. 
The results we present here allow us to propose a model for the transmission of mtDNA (Fig. 5). The high mtDNA copy number in mature oocytes ( 175,000 copies, likely organized at 1 to 2 copies per organelle) can be viewed as a genetic device to ensure distribution of mitochondria to the cells of the early embryo at a time when mitochondrial biogenesis is arrested. Resumption of mitochondrial biogenesis by E6.5 results in a 10-20 fold increase in mtDNA copy number from the emergence of primordial germ cells until colonization of the gonad at E13.5. While there is no measurable segregation of neutral sequence variants at this time, a physical bottleneck ( 200 mtDNAs) in the earliest PGCs enables selection against severely deleterious mtDNA mutations at the level of the organelle, possibly through replicative competition ${ }^{131}$. The genetic bottleneck for neutral (and less deleterious mtDNA sequence variants that are associated with most human disease) occurs during folliculogenesis, in early postnatal life, due to replication of a subpopulation of mtDNAs. In this way, mutations that escape the filter for purifying selection are rapidly segregated and exposed to selection at the level of the organism in individual maternal lineages. It remains to be determined how a subpopulation of mtDNAs is preferentially replicated in early postnatal oocytes, but the mechanism could involve turning off replication in the majority of organelles. 


\section{Materials and Methods}

\section{Transgenic Mice}

We obtained mice carrying the GOF18deltaPE transgene, which is a specific marker of PGCs after E8.5, from Dr. H. Scholer ${ }^{123}$. The males (C57BL/6 background) were crossed to Balb/c heteroplasmic females ${ }^{4}$. The F3 GOF18deltaPE+/+ males were backcrossed to F2 GOF18deltaPE+/+ heteroplasmic transgenic females, generating the OG2-BALB/NZB line. Genotyping for the presence of the transgene was performed as described ${ }^{123}$. Sex genotyping was performed as described ${ }^{132}$. The McGill University Animal Care Committee approved the animal studies.

Isolation of cleavage stage embryos, oocytes, single embryonic germ cells.

Early embryos (E0.5 to E3.0) were flushed from oviducts following natural timed matings of OG2-BALB/NZB mice. Postnatal oocytes were harvested from individual follicles using tungsten needles. Female PGCs were isolated from OG2-BALB/NZB E8.5 to E14.5 embryos by disaggregating the embryo under an epifluorescencent dissecting microscope and manually pipetting individual germ cells expressing GFP. Disaggregation was performed by enzymatic digestion with Collagenase type 1A (Sigma) and Trypsin-EDTA (0.25\%) in a 1:1 ratio (total volume: $50 \mathrm{ul}$ ) at $37 \mathrm{C}$ for 5 minutes, inverting the microfuge tube every 30 seconds. Single cell separation was achieved by pipeting up and down and by vortexing briefly. Enzymatic digestion was arrested by addition of a 10-fold excess of cold PBS $+0.01 \%$ BSA. Individual cells or embryos were washed three 
times in $\mathrm{Ca}^{2+}$ - and $\mathrm{Mg}^{2+}$ - free PBS and finally transferred into a PCR tube containing 10ul of the washing solution. The cells were lysed by three rounds of freeze- thawing (heating at 99C with immediate freezing in liquid N2) as previously described ${ }^{133}$. Freeze-thawing in PCR buffer or buffers containing detergents had a deleterious effect on the amplification efficiency of the MT9/11 Q-PCR assay.

\section{Mitochondrial DNA Quantitation by Quantitative Real-Time PCR (Q-PCR).}

Single cell lysates were used as the template and Q-PCR reactions were performed in triplicate using MT9 Forward (5'GAGCATCTTATCCACGCTTCC3') and MT11 Reverse (5'GGTGGTACTCCCGCTGTAAA3') primers (Supplementary Fig. 2). Absolute mtDNA copy number in a 1 ul aliquot of single cell lysates was calculated using a standard curve derived from Q-PCR amplifications of 10 to $10^{6}$ copies of a region of mtDNA subcloned into PCR2.1-TopoTA vector using primers MT12 (5'CGCCCTAACAACTATTATCTTCC3') and MT13 (5'GACCGTTTGTTTGTTGTTGAAA3').

The plasmid standard was constructed by cloning a region of mtDNA amplified using primers MT12 (forward) and MT13 (reverse) followed by cloning into PCR2.1-TopoTA (Invitrogen). The MT12/13 plasmid standard concentration was determined by UV spec and High Mass ladder/Agarose gel. Q-PCR reactions using MT9 (forward) and MT11 (reverse) primers were run using DNA Faststart Master Plus SYBR Green (Roche) on the Rotorgene 3000 (Corbett) according to the following conditions: 35 cycles, denaturation at $95 \mathrm{C}$ for 10 seconds, 
annealing at $60 \mathrm{C}$ for 15 seconds, and extension at $72 \mathrm{C}$ for 20 seconds. Melt curve analyses reported on the specificity of the PCR products that were amplified. Post-run analyses of amplification efficiency were performed and corrected using Rotorgene software and LINREGPCR ${ }^{134}$ programs. All samples and standards were performed in triplicate using $1 \mathrm{ul}$ of template per $10 \mathrm{ul} \mathrm{PCR}$ reaction. This Q-PCR system could detect as few as ten copies of a standard DNA template and the linear regression analysis of all standard curves for sample with copy number between 10 and $10^{9}$ showed a high correlation coefficient $\left(r^{2}=\right.$ 0.999). The Q-PCR assay was validated by slot blotting serially diluted MT12/13 plasmid standards as well as pooled ovulated oocytes from OG2-BALB/NZB females (data not shown). Rho zero cells were as negative controls.

\section{Mitochondrial DNA Genotyping}

The proportion of NZB mtDNA in single cells was determined essentially as previously described ${ }^{135}$ except that non-radioactive nested PCR reactions were first performed using primers MT12 (forward) and MT13 (reverse) for 20 cycles in single cell lysates obtained from embryonic and neonatal germ cells. $2 \mathrm{ul}$ of these PCR products were subsequently amplified using MT9 and MT10 (5'CTGCTTCAGTTGATCGTGGGT3') in a "last cycle hot" PCR reaction. 


\section{BrdU Labeling}

Neonatal females (P1 to P4) were subcutaneously injected with $100 \mathrm{mg} / \mathrm{kg}$ body weight BrdU (Roche) and sacrificed after 2 or 24 hours. Tissues were immediately harvested and prepared for immunohistochemistry.

\section{Confocal Microscopy and Immunohistochemistry}

For immunocytochemistry, C2C12 mouse cells were grown in DMEM (Hyclone) containing 10\% fetal bovine serum and plated on coverslips prior to fixation in $4 \%$ paraformaldehyde. For immunohistochemistry, tissues harvested from BrdU-injected pups were frozen in OCT freezing medium. Cryosections (10 $\mu \mathrm{m})$ were incubated with various combinations of: mouse anti-BrdU (Roche) (1/10), mouse anti-GM130 (1/250) (BD Biosciences Pharmingen), rabbit antiTFAM (gift of Dr. B. Kaufman), anti-mtSSB (gift of Dr. M. Zeviani), anti-POLG (gifts of Dr. W. Copeland) primary antibodies (1/250), followed by anti-mouse (goat) Alexa488 and anti-rabbit (goat) Alexa568 (both 1/500) (Molecular Probes, Eugene, OR). Parallel negative controls omitted the primary antibodies. In some instances a series of fluorescent confocal images ( $Z$ stacks) were acquired with a LSM 510 meta confocal microscope (Zeiss Oberkochen,Germany) and visualized using a 543-nm Helium-Neon laser and 488-nm Argon laser lines respectively. Slides were imaged in $Z$ series every $0.20 \mu \mathrm{m}$ and $Z$ images were deconvolved according to empirically determined point spread functions with Huygens Professional 3.0 software. 


\section{Transmission Electron Microscopy and Immunohistochemistry}

Ovaries taken from neonatal mice injected with BrdU (see above) were fixed and processed in either Epon (Hexion Specialty Chemicals) or LR White acrylic resin (London Resin Company; Berkshire, UK) as previously described ${ }^{136}$. For transmission electron microscopy (TEM), selected regions were trimmed, and ultrathin sections $(80 \mathrm{~nm})$ were placed on Formvar-coated nickel grids. Postembedding immunolabeling was performed on the grid-mounted sections as described previously ${ }^{136}$. Grid-mounted tissue sections were processed for colloidal-gold immunocytochemistry by incubation of the sections with either undiluted anti-BrdU (Roche) or anti-Tfam primary antibodies, after which immunolabeling patterns were visualized by incubation with protein A-colloidal gold complex (18 nm; Dr. M. McKee, McGill University, Montreal, Canada). Briefly, the grids were incubated for 10 min with $1 \%$ BSA in PBS, followed by incubation with primary antibody for $1 \mathrm{~h}$, blocking with $1 \% \mathrm{BSA}$ in PBS, and then incubation with protein A-gold for 30 min. The grids were then rinsed with distilled water, air-dried, and stained conventionally for transmission electron microscopy using uranyl acetate and lead citrate. The images were recorded using a Philips FEI Tecnai 12 transmission electron microscope operating at $120 \mathrm{kV}$.

\section{Statistical Testing}

The non-normal (beta) heteroplasmy distributions in germ cells, embryos, or offspring violate the assumptions of the parametric F-test for equal variances. Hence we used the Levene Test and Bootstrapped-Levene Test for Equal 
Variances using the Brown and Forsythe correction ${ }^{137}$. Head to head comparisons of all known tests of equal variances have shown that the Levene test is the most robust and powerful, and least likely to commit type 2 errors 138,139. Nevertheless, we tested the power and robustness of both the bootstrapped and non-bootstrapped Brown-Forsythe corrected Levene tests, validating them both by the Monte Carlo permutation test on simulated data as well as on actual data sets wherein previously established differences in genotypic variance had been proven ${ }^{140}$. The results indicated that in the event of small sample sizes (i.e. $n>5$ ), the bootstrapped version of the Levene test is more reliable. Pairwise comparisons of the genotypic variance of groups of cells sampled at various points during germline development were performed using both the bootstrapped and non-bootstrapped Levene tests and $p$ values are reported in table S1 (Supplementary Fig. 1).

\section{Acknowledgements}

We thank Louis Villeneuve for help with confocal microscopy, Jeannie Mui, for assistance with electron microscopy, Timothy Johns, Fiona Jones, and Davood Sabour for technical assistance, and José Correa for statistical design. We are grateful for antibodies directed against TFAM (Dr. B.Kaufman), mtSSB (Dr. M. Zeviani), and POLG (Dr. W. Copeland). The OCT4 PE-EGFP mice were obtained from Dr.H.R. Scholer. This research was supported by the Canadian Institutes of Health Research and the National Institutes of Health (USA). EAS is an International Scholar of the HHMI. 


\section{Figure Legends}

\section{Fig 1. Changes in mtDNA copy number during female germline}

\section{development.}

Top panel shows mean mitochondrial DNA copy number $( \pm$ SEM) measured in single embryonic germ cells isolated from OG2-BALB/NZB female embryos from E8.5 to E16.5. Bottom panel shows mean mitochondrial DNA copy number ( \pm SEM) measured in single postnatal oocytes isolated from live female offspring born to OG2-BALB/NZB mothers. Most of the oocytes from the P1-P4 animals are in primary follicles, and most from the P29 animals are in secondary follicles. The insets show the frequency histograms of heteroplasmy levels in primary oocytes from a P1 mouse (left) and a P29 mouse (right), demonstrating the change in genotypic variance that is due to the mitochondrial genetic bottleneck.

\section{Fig. 2. Replicating mtDNA visualized in vivo by incorporation of BrdU.}

BrdU incorporation studies in primary oocytes labeled for (A) 24 hours or (B) 2 hours show that replicating mtDNAs (green) represent a small subgroup of all Tfam-positive mtDNA nucleoids (red). BrdU labeling in sections of neonatal heart (C) and in sections of neonatal liver (D) are shown for comparison. The degree to which BrdU punctae colocalize with Tfam punctae is tissue-specific. Sections of primary oocytes show the least colocalization compared to neonatal heart or liver sections. Confocal micrographs are represented as merged maximum projections. Insets to the right in each panel show close-ups of individual channels and merged maximum projections. Videos showing 3D views of the 
merged maximum projection close-ups for primary oocytes and neonatal heart are shown in Supplementary data (Video S1, primary oocyte, Video S2, neonatal heart).

\section{Fig. 3. The distribution of mtDNA nucleoids in primary oocytes.}

(A) Tfam-positive mtDNA nucleoids (red) are asymmetrically distributed about the GM130-positive Balbiani body (green) in primary oocytes. By the secondary follicle stage $(B)$, oocytes regain their homogeneous distribution following the dispersion of the Balbiani body. Confocal micrographs are presented as merged maximum projections.

Fig. 4. Transmission EM and immunogold labeling in primary oocytes. Mitochondria are asymmetrically distributed about the Balbiani body (A) (x6000) and are cristae poor (B) (x10 000). No more than 2 foci of either BdrU (C) or Tfam (D) were observed in cross-sections of oocyte mitochondria after immunogold labeling (x 43000$)$.

\section{Fig. 5. Model for the Transmission of mtDNA in the female germline.}

The postnatal mitochondrial genetic bottleneck during folliculogenesis rapidly segregates sequence variants that pass through the filter for purifying selection at the physical bottleneck for mtDNA in primordial germ cells. 


\section{Supplementary Information}

Supplementary Fig. 1: Pairwise comparisons of mtDNA genotypic variance using the Brown-Forsythe corrected Levene and Bootstrapped Levene Tests.

All possible pairwise comparisons of genotypic variances generated from groups of $(A)$ embryonic and $(B)$ postnatal germ cells were made. The resulting $p$ values for both non- parametric tests of equal variance are listed in each case, with red fill in the cases where a statistically significant difference in genotypic variance was discovered $(p<0.05)$.

\section{Supplementary Fig.2. BrdU incorporation in cells and tissues labels replicating mtDNA nucleoids.}

BrdU incorporation in $\mathrm{C} 2 \mathrm{C} 12$ mouse myoblast cells in the presence of Aphidicolin (which inhibits replication of nuclear DNA) labels replicating mtDNA. BrdU punctae (green) colocalize with TFAM (A), mtSSB (D), and POLG $(G)$, which are labeled red. Compared to the in vivo BrdU incorporation of cardiomyocytes costained for TFAM (B), mtSSB (E), or POLG $(H)$ and primary oocytes co-stained for TFAM (C), mtSSB (F), or POLG (I), cultured mouse myoblasts show greater degrees of colocalization of the nucleoid protein markers with BrdU immunoreactivity. Confocal micrographs are represented as maximum projections. 
Supplementary Fig. 3. Strategy for mtDNA Heteroplasmy Quantification. Nonradioactive nested PCR using MT12 (forward) and MT13 (reverse) primers was followed by a "last cycle hot" PCR using MT9 (forward) and MT10 (reverse) primers. Fragments of digested PCR products separated by electrophoresis are quantified by densitometry. MT9 (forward) and MT 11 (reverse) primers are used absolute copy number measurements made by Quantitative Real Time PCR.

Supplementary Fig. 4. Immunocytochemical analysis of the relationship between BrdU positive foci and the Balbiani body in postnatal oocytes. The Balbiani body (anti-GM130) and BrdU are labeled in green and TFAM is labeled red. 


\section{Supplementary Videos.}

$3 \mathrm{D}$ views of the merged maximum projection close-ups in

Figure 2 for primary oocytes (Video S1) and neonatal heart (Video S2).

Supplemental Videos 1 and 2 can be found on the Nature Genetics website:

http://www.nature.com/ng/journal/v40/n12/suppinfo/ng.258 S1.html 
Figure 1
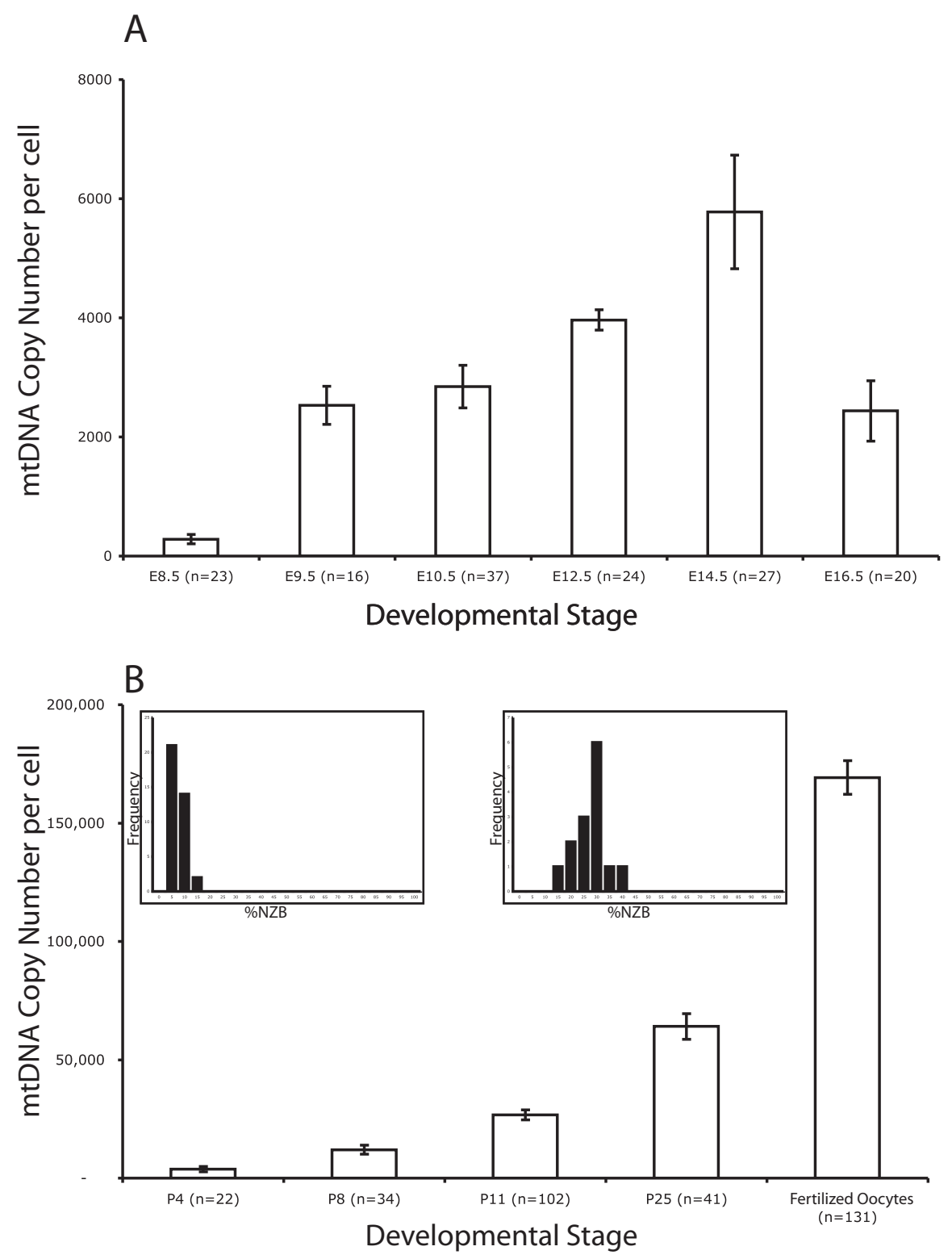
Figure 2
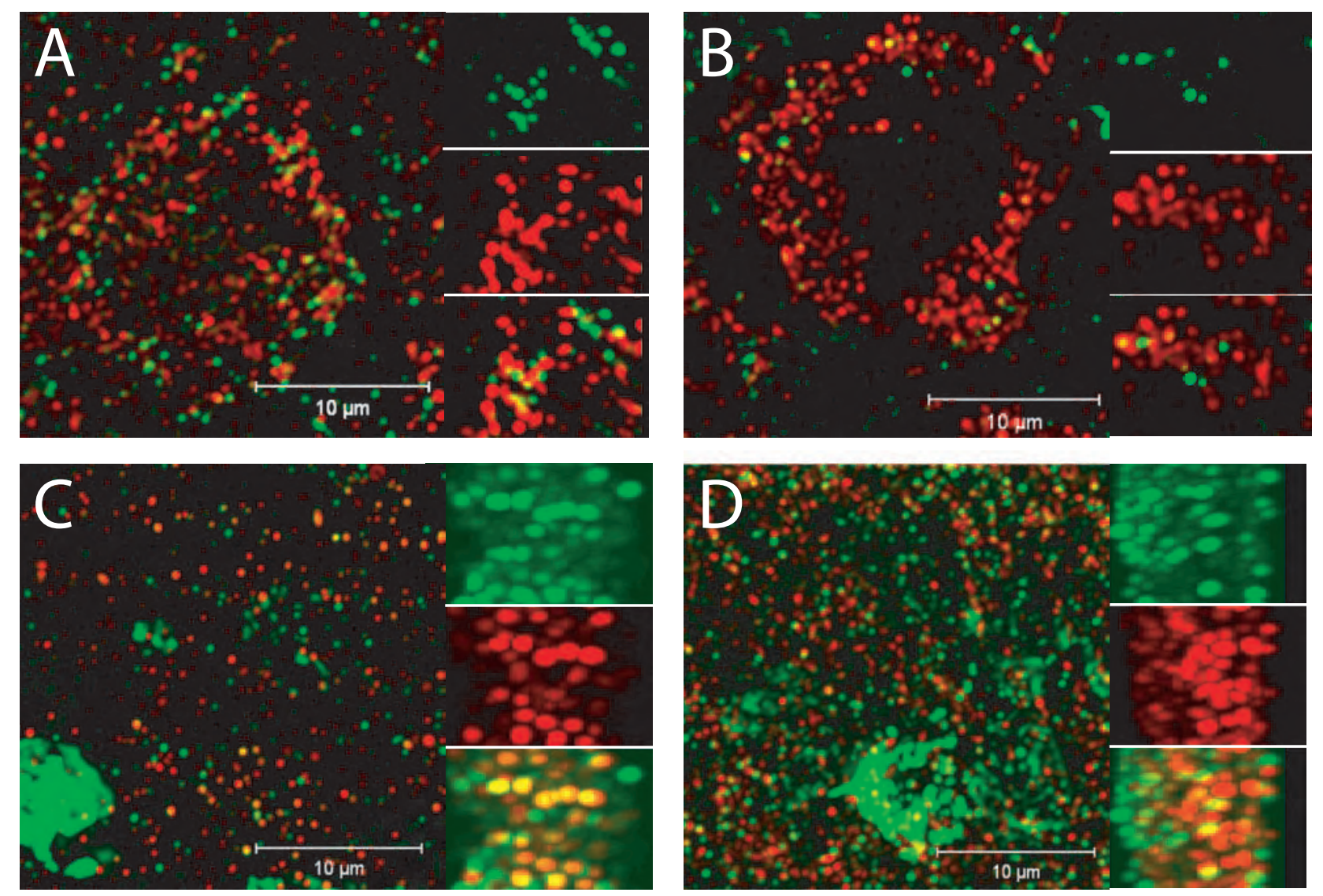
Figure 3




Figure 4








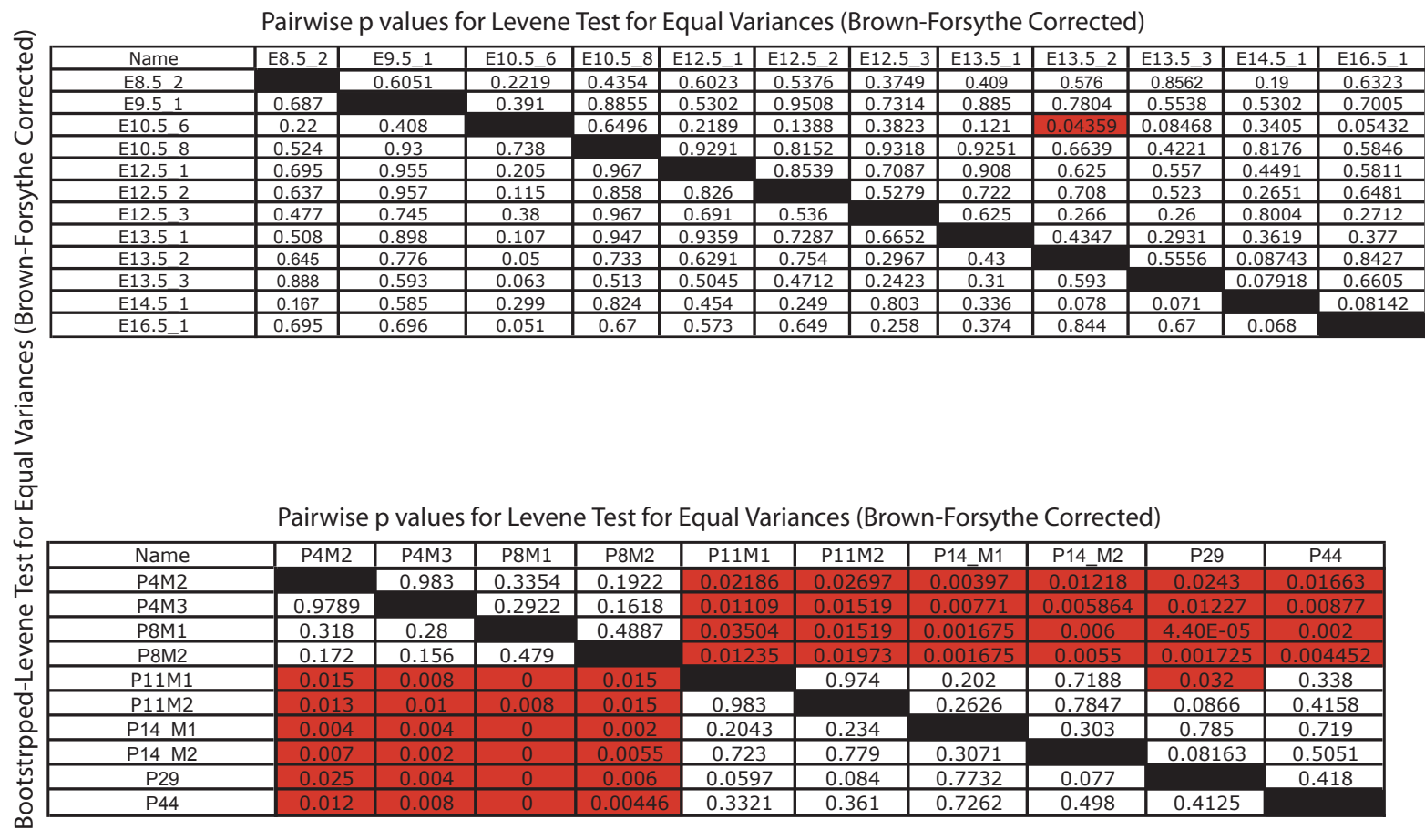




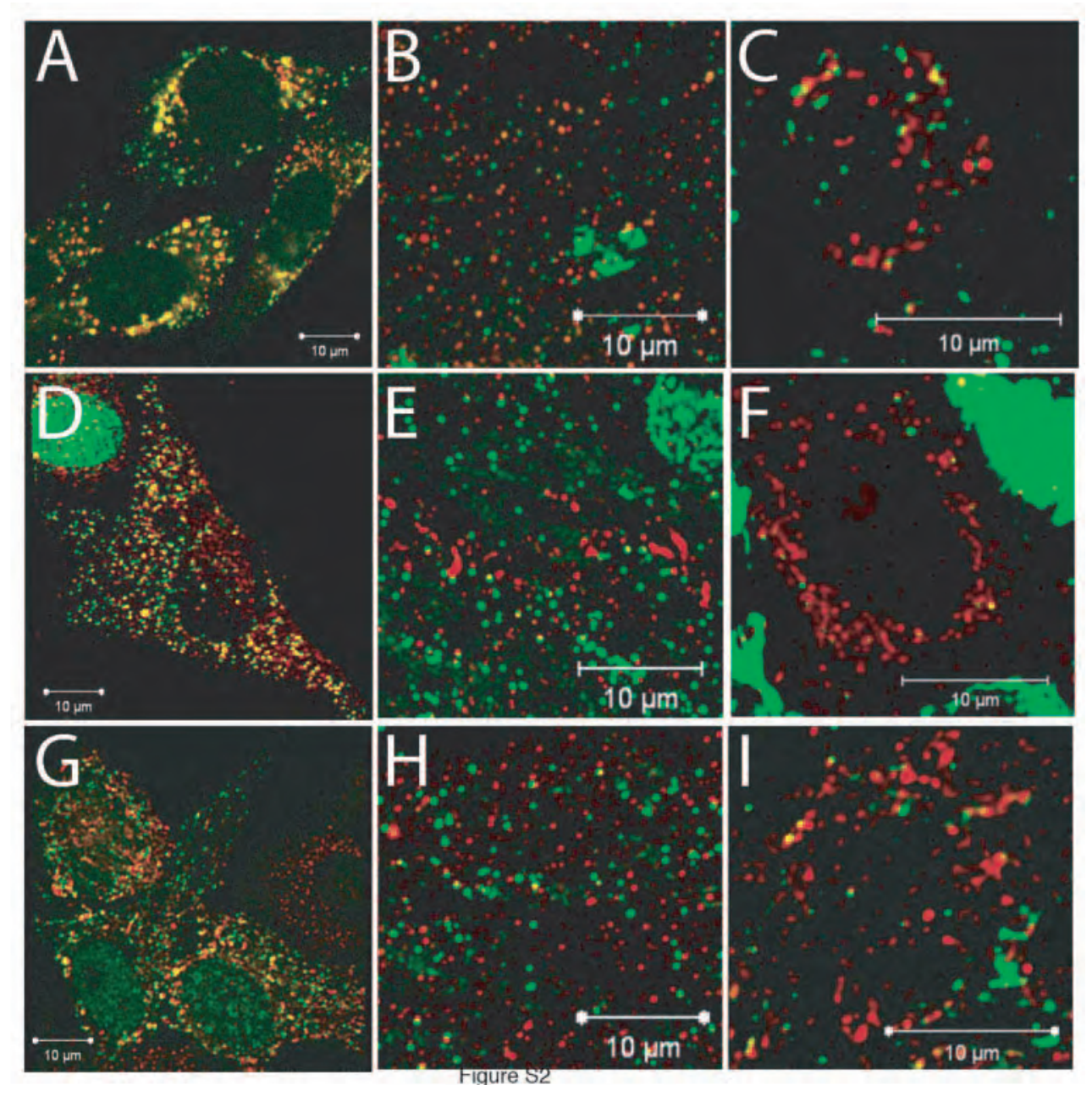




\section{MTDNA HETEROPLASMY}

NESTED PCR

ROUND 1: MT12 \& MT13 PRIMERS

ROUND 2: MT9 \& MT10 PRIMERS

AdD ${ }^{32}$ P PRIOR To Final CyCLE

RFLP ANALYSIS ON PAGE

QUANTIFICATION BY PHOSPHOIMAGER

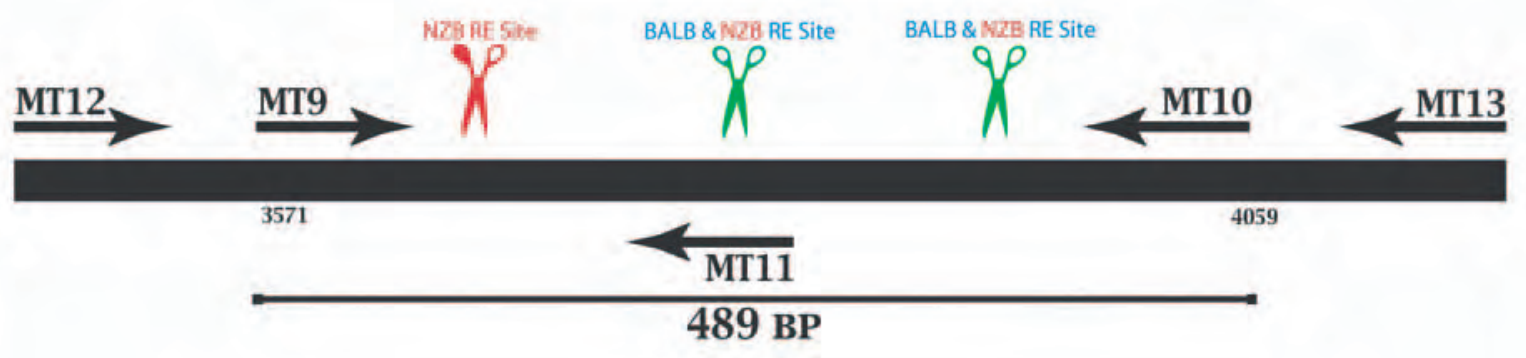

Sample

$\# 1 \quad \# 2$

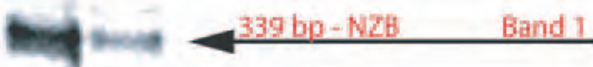

t218bp-BALB Band 2

$-121 \mathrm{bp}-\mathrm{BALB}$

Band 4

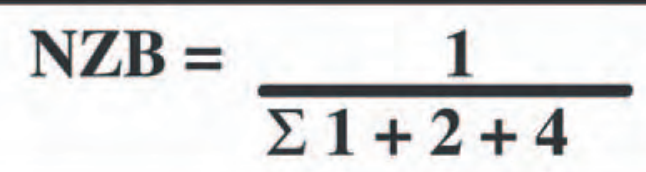




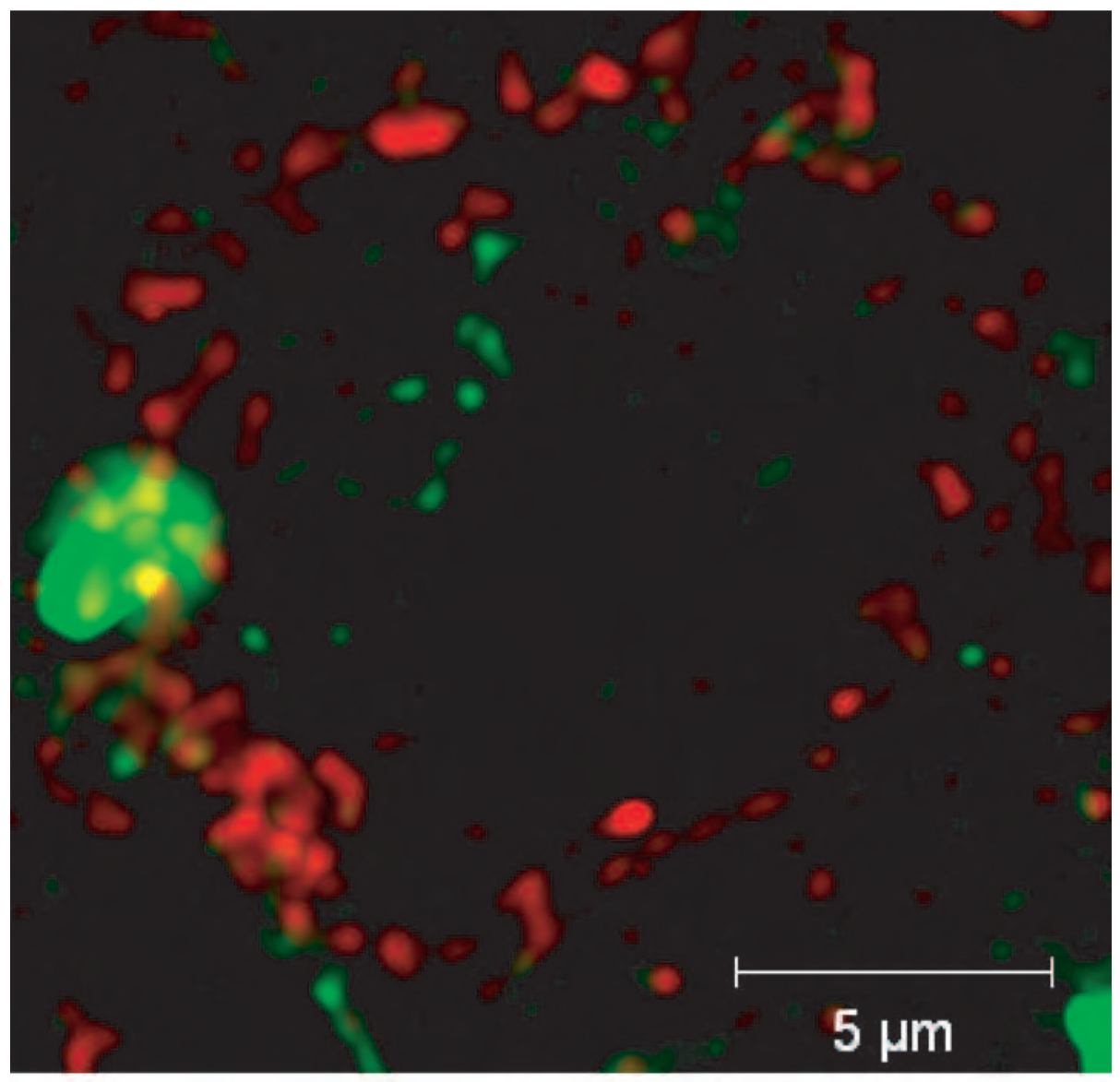

FIGURE S4 
Supplementary Table 1. Analysis of heteroplasmy variance in oocytes from postnatal mice.

\begin{tabular}{|c|c|c|c|c|c|c|c|c|c|c|c|}
\hline $\begin{array}{c}\text { Postnatal } \\
\text { Stage }\end{array}$ & \begin{tabular}{|c|} 
Mean \\
heteroplasmy \\
level $(\%)$
\end{tabular} & $\begin{array}{c}\text { Standard } \\
\text { Error }\end{array}$ & Median & $\begin{array}{l}\text { Standard } \\
\text { Deviation }\end{array}$ & $\begin{array}{c}\text { Sample } \\
\text { Variance }\end{array}$ & Kurtosis & Skewness & Range & Minimum & Maximum & Count \\
\hline P4_1 & 21.7 & 0.6 & 21.8 & 1.6 & 2.6 & 0.5 & -0.2 & 5.3 & 19.0 & 24.3 & 8.0 \\
\hline P4_2 & 20.2 & 0.6 & 19.9 & 1.9 & 3.6 & 2.4 & 0.9 & 7.3 & 17.1 & 24.3 & 10.0 \\
\hline P8_1 & 5.1 & 0.4 & 4.5 & 2.5 & 6.5 & 2.3 & 1.2 & 12.4 & 1.1 & 13.6 & 37.0 \\
\hline P8_2 & 3.9 & 0.7 & 3.1 & 3.2 & 10.3 & 3.4 & 1.7 & 13.9 & 0.2 & 14.2 & 24.0 \\
\hline $\mathrm{P} 1 \overline{1}_{-} 1$ & 26.5 & 0.8 & 26.8 & 5.6 & 31.2 & 0.6 & 0.1 & 28.8 & 11.7 & 40.5 & 46.0 \\
\hline $\mathrm{P} 11 \_2$ & 16.5 & 1.2 & 15.8 & 5.6 & 31.7 & 0.3 & 0.4 & 21.8 & 7.3 & 29.1 & 23.0 \\
\hline P14_1 & 28.3 & 2.7 & 27.0 & 7.7 & 58.6 & -1.0 & 0.6 & 20.3 & 20.5 & 40.8 & 8.0 \\
\hline P14_2 & 15.1 & 1.1 & 15.0 & 5.8 & 33.6 & -0.1 & -0.1 & 23.5 & 2.7 & 26.2 & 30.0 \\
\hline P29 & 41.0 & 1.7 & 43.2 & 9.7 & 94.7 & 2.0 & -1.1 & 45.1 & 12.6 & 57.7 & 33.0 \\
\hline P30 & 26.8 & 1.9 & 27.6 & 9.1 & 83.7 & -0.8 & -0.2 & 31.3 & 10.9 & 42.2 & 24.0 \\
\hline P44 & 25.4 & 1.9 & 26.8 & 7.0 & 48.4 & -0.4 & -0.1 & 24.6 & 13.3 & 37.9 & 14.0 \\
\hline
\end{tabular}


Supplementary Table 2. Sequences of the primers used in this study.

\begin{tabular}{|l|l|}
\hline Primer & Sequence 5' to 3 ' \\
\hline MT11 & GGTGGTACTCCCGCTGTAAA \\
\hline MT9 & GAGCATCTTATCCACGCTTCC \\
\hline MT10 & CTGCTTCAGTTGATCGTGGGT \\
\hline MT12 & CGCCCTAACAACTATTATCTTCC \\
\hline MT13 & GACCGTTTGTTTGTTGTTGAAA \\
\hline
\end{tabular}


Chapter 2 is a manuscript to be submitted for publication, which tests the model for the transmission of mitochondrial DNA sequence variants put forth in Chapter 1. The analysis of segregation patterns in heteroplasmic early and late germlinespecific conditional knockout mouse models that affect mitochondrial DNA copy number and OXPHOS function support the model of a post-natal mitochondrial genetic bottleneck. In this Chapter I adapt a population genetics equation to determine the size of the mitochondrial genetic bottleneck in the germlines of various wild-type and conditional knockout females. 


\section{Chapter 2}

\section{The size of the mitochondrial genetic bottleneck}

Timothy Wai, Brendan J. Battersby, and Eric A. Shoubridge 


\section{Abstract:}

For more than a decade it has been known that the germline segregation of neutral mtDNA sequence variants is a consequence of random genetic $\mathrm{drift}^{3,4}$ and we recently showed that the genetic bottleneck responsible for the rapid segregation of mtDNA in the female germline of the mouse is caused by replication of a small subgroup of mitochondrial genomes in the primary oocytes of the developing postnatal ovary ${ }^{141}$, although the spatial resolution achieved by current light microscopy cannot accurately determine the number of replicating genomes. Unexpectedly, the physical bottleneck, caused by the absence of mtDNA replication prior to oogenesis did not significantly contribute to the segregation of mtDNA sequence variants following the resumption of mitochondrial biogenesis in the primordial germ cell population. By modifying a population genetics model that predicts the rate of allelic fixation, we are able to use the evolution of genotypic variance and mtDNA copy number in the developing female germline to estimate the size of the subgroup of replicating templates at the mitochondrial genetic bottleneck. We validated our model using early and late germline-specific deletion of TFAM and COX10 in our previously established heteroplasmic mouse mode ${ }^{141}$. Further constriction of the physical bottleneck by early germline-specific deletion of TFAM leads to an increase in genotypic variance during embryogenesis that is subsequently amplified by the post-natal bottleneck to give a heteroplasmic distribution with large variance. Finally, comparisons of mouse and human segregation patterns indicate that the genetic bottleneck in humans is markedly narrower than in mice. 


\section{Introduction:}

For the last 25 years it was believed that the mtDNA genotypic variance observed in the offspring of heteroplasmic animal models, and in human pedigrees, was principally due to the creation of a genetic bottleneck during embryogenesis. A reduction in mtDNA copy number followed by the rapid amplification of this restricted pool of templates would, it was thought, generate most of this genotypic variance during the first few mitotic divisions of the primordial germ cells ${ }^{2-5}$. This idea was recently dispelled when we failed to detect significant fluctuations in genotypic variance during embryonic germline development ${ }^{141}$. After tracking the evolution of genotypic variance throughout postnatal germline development we arrived at the inescapable conclusion that the mitochondrial genetic bottleneck results from the replication of a small subgroup of mitochondrial genomes in the early postnatal oocyte ${ }^{141}$. While these genetic data were corroborated by the visualization of a fraction of mitochondrial genomes undergoing replication, technological limitations, including the spatial resolution of traditional confocal microscopy, prevented an accurate estimate of the size of this replicating subgroup.

In order to determine the number of segregating units (i.e. the size of the mitochondrial genetic bottleneck) we have employed a population genetics model that incorporates the evolution of genotypic variance and mtDNA copy number we observe in the developing female germline. This new prediction of bottleneck size in the germline also accounts for the segregation pattern in our collection of 
heteroplasmic lines of mice, which, to our knowledge, represents the largest data set of its kind.

Using lines of heteroplasmic mice segregating BALB and NZB mtDNA on different nuclear genetic backgrounds, including M.m. castaneus and M.m. domesticus, we demonstrate that the bottleneck size is variable between and within sub-specific maternal lineages. We show that the rate of segregation can be increased when mtDNA copy number is reduced in the oocytes before they pass through the bottleneck. Finally, we compare the segregation rates of various common human pathogenic mutations and neutral mtDNA sequence variants and conclude that the size of the genetic bottleneck is not only subject to biological variability within a species, but also differs between mice and humans. 


\section{Results:}

\section{Estimating the proportion of replicating templates in the female germline}

While random genetic drift shapes the distribution of heteroplasmy in the offspring of the next generation, the rate of homoplasmic fixation (i.e. the rate of segregation) is determined by the size of the genetic bottleneck (also defined as effective population size or number of segregating units). In order to calculate the size of the mitochondrial genetic bottleneck, we returned to a population genetics model originally conceived by Sewell Wright, and later adapted by Solignac and coworkers to calculate the rate of segregation of mitochondrial DNA in flies ${ }^{142}$. We had originally used this equation, where the underlying assumptions of this derivation are non-overlapping generations, no selection, no migration and no denovo mutation, in a finite and steady population size, to calculate the effective number of segregating units in heteroplasmic mice segregating BALB and NZB $m t_{D N A}$. The calculation of genotypic variance can be written is a function of three parameters; $00, t$ and Neff. Neff represents the number of segregating units while the initial maternal heteroplasmy level, which is equal to the mean of that in the offspring, is defined as $\mathrm{p} 0$, and $\mathrm{t}$ is the number of generations the Neff goes through to reach the final population size.

$$
V=p 0(1-p 0)\left[1-(1 / \text { Neff })^{t}\right]
$$

While it is incorrect to assume either a steady or finite population size, such an assumption simplifies our calculations and, fortunately, has a negligible impact on the estimation of Neff ${ }^{143}$. Originally, the number of generations ( $t$ ) had been set to 15 to reflect the number of mitotic divisions required to generate the mature 
murine oocyte ${ }^{4}$. Until recently, it was believed that the replication of a restricted pool of mtDNAs, particularly in the early stages of oogenesis, was responsible for generating the vast majority of the genotypic variance observed in the offspring of the next generation, but we now know that only a subgroup of replicating templates in the postnatal ovary of the mouse is responsible for the intergenerational genetic bottleneck ${ }^{141}$. Hence, we must re-evaluate the parameters of the Sewell-Wright equation if we are to accurately determine the actual size of the genetic bottleneck.

By tracking the evolution of genotypic variance and mtDNA copy number in the developing female germline, we are able to calculate the number of segregating units (Neff) predicted to yield a given genotypic variance, which is calculated by averaging the squared difference of an observed heteroplasmy level from the group mean (maternal) level. However, there is a confounding factor here: the normality of these heteroplasmy distributions is greatly affected by the mean level of heteroplasmy, and boundary conditions of fixation will inevitably result in non-normal (beta) distributions when maternal heteroplasmy levels are not in the mid- range (Figure 1).

Hence, heteroplasmy levels tending toward either BALB or NZB homoplasmy result in a low variance whereas mean heteroplasmy, near 0.5 , yields the maximum measured variance (Figure 2A). Standard population genetics theory says that this confounding factor can be corrected for by normalizing the heteroplasmy variance by $\mathrm{p} 0(1-\mathrm{p} 0)$, where $\mathrm{p} 0$ is the group mean or maternal heteroplasmy level (Figure 2B). 
So, the original Sewell-Wright equation

$$
V=p 0(1-p 0)\left[1-(1 / \text { Neff })^{t}\right]
$$

can be re-written as follows:

$$
\mathrm{V} /(\mathrm{p} 0(1-\mathrm{p} 0))=1-(1 / \mathrm{Neff})^{\mathrm{t}}
$$

where $\mathrm{V} /(\mathrm{p} 0(1-\mathrm{p} 0))$ represents the normalized variance.

So, for any group of cells sampled during germline development, it is now possible to solve for Neff and t when starting with a measured variance and maternal heteroplasmy without generating any bias (Figure 2C). But since Neff and $t$ are and not independent variables, how do we decide which precise values to substitute? (Recall that the number of generations was initially set to reflect the number of mitotic divisions of the required to produce a mature oocyte: $t$ was held constant at 15, making the calculation of Neff rather straightforward). For the purposes of simplification, we begin with the assumption that the mitochondrial genome represents the segregating unit. For each sample, we empirically adjust the value of Neff in relation to $t$, which now represents the number of replications of mtDNA, by calculating the precise Neff and t values that, according to the equation Neff $X 2^{t}$, would yield a final mtDNA copy number equal to the mean mtDNA copy number of that sample. Implicit to this model are assumptions of a constant number of genomes per nucleoid during the development of the germline. 
Until now, the importance of empirically determining the value of $t$ has been ignored in the calculation of bottleneck size $e^{4,5,143}$ because it was assumed that all mtDNAs in a developing germ cell were replicating at equivalent rates. Having demonstrated that this is not true in the postnatal ovary, we sought to calculate the effective number of segregating units responsible for generating the genotypic variance in the context of a known content of mtDNA for various stages of germline development. We observe that the normalized genotypic variance increases dramatically in postnatal life, after the transition from primary to secondary follicles (Figure $3 \mathrm{~A}$ ) and of course in mature oocytes (Figure 3B) and offspring (Figure 3C). Congruent with the results of our non-parametric tests of equal variances ${ }^{141}$, we do not observe an increase in normalized genotypic variance during embryonic germline development and so we are able to estimate the number of segregating units, which we equate to the number of mitochondrial genomes estimated to replicate at every point in germline development (Table 1).

Based on the observed mean mtDNA content of the cells of the developing germline, we were able to determine the number of segregating units (i.e. mtDNA templates) required to replicate in order to yield a given normalized genotypic variance. As the germline mtDNA copy number increases, the proportion of genomes originally designated to replicate in order to repopulate the germline decreases precipitously. (Figure 4, Table 1). Hence, we conclude that the majority of the observed genotypic variance in the oocytes of the post-natal ovary is generated by the replication of fewer than $5 \%$ of the mtDNAs present in the oocyte of the primary follicle ( 13,000 copies). It must be said, however, that 
while the variance measured during embryonic oogenesis does not rise to a level of detection that is statistically significant according to non-parametric test of equal variances ${ }^{141}$, we cannot rule out that the genotypic variance present prior to the post-natal genetic bottleneck does not in some way contribute to an increase rate of segregation once the subgroup of replicating genomes is selected in the primary oocyte. Nevertheless, we conclude that the mitochondrial genetic bottleneck is defined by the replication of a subgroup of genomes composing fewer than $2 \%$ of the all of the mtDNAs in the primary oocyte contained in a preantral secondary follicle ( 26,000 copies). In other words, the vast majority of mtDNAs in the mature oocyte ( 161,000 copies) originated from a minority of templates in the primary follicle; a fraction that equates to less than $0.1 \%$ of the total content of mtDNA in the mature oocyte.

\section{Estimating the Size of the Bottleneck}

The comparisons of genotypic variances, made using validated nonparametric tests of equal variances (e.g. Brown-Forsythe corrected Bootstrapped Levene test ${ }^{137}$ do not provide a quantitative description of the degree of difference between maternal lineages, particularly when the maternal heteroplasmy levels are dramatically different (Figure 1). We use our model to estimate the size of the bottleneck responsible for shaping the distribution of heteroplasmy using a large number of maternal lineages with varying maternal heteroplasmy levels. We report that the mean bottleneck size in our wild type heteroplasmic mice $(n=687)$ is 189 segregating units (Figure 5), (range 90 to 
325). When we analyzed the segregation patterns of our heteroplasmic mice either by non-parametric tests of equal variances or by the size of the genetic bottleneck, we observed a great deal of variability in the number of segregating units (Figure 5, Table 2). Since we also observed substantial variation in the amount of mtDNA present in the oocytes and early embryos $(161,000 \pm 73,000$ copies. Chapter 3, Figure 1) as well as in germ cells sampled during embryonic stages of oogenesis (Figure S1), we propose that the natural variability in size of the genetic bottleneck, caused by the replication of a subgroup of mitochondrial genomes, may simply reflect the natural variability in the number of copies of mtDNA present during oogenesis and, by extension, the start of embryogenesis.

\section{Germline segregation of mtDNA is not affected by nuclear genetic}

\section{background}

It has been shown that BALB and NZB mtDNA genotypes exhibit clear somatic tissue-specific segregation patterns ${ }^{144}$, which were later attributed to nuclear genetic background differences between two sub-species of mice; M.m. domesticus and M.m. castaneus ${ }^{140}$. While there is no directional selection in the germline of these mice, we sought to test whether such nuclear genetic background differences could alter the rate of germline segregation. We compared the segregation pattern in offspring generated from females covering a wide range of heteroplasmy levels (initial allele frequencies $10 \%$ to $80 \%$ ) that were segregating on either domesticus or castaneus nuclear backgrounds (Figure 1, Table 3). We did observe a very few significant differences in 
genotypic variance among litters of either domesticus or castaneus females (Table S1), which may reflect small sample sizes of the individual litters. We observed significant differences in genotypic variance within domesticus (Table S2A) and castaneus (Table S2B) maternal lineages but did not observe differences in mean bottleneck size between these two sub-species. Given that there is just as much variability in bottleneck size within a sub-species as there is between sub-species, we are unable to conclude that sub-specific differences in nuclear genetic background between domesticus and castaneus contribute in any way to the rate of germline segregation. Using the largest reported data set to date, ( $n \approx 1500$ offspring), we confirm that germline segregation pattern of BALB/NZB mtDNA is due entirely to random genetic drift, and is consistent with heteroplasmic distributions described by the Kimura equation ${ }^{143}$ (data not shown). 


\section{Genetically Manipulating the Bottleneck}

Since the replication of a subgroup of mitochondrial genomes is responsible for the rapid segregation of BALB/NZB genotypes, a decrease in the number of replicating genomes at this genetic bottleneck would be predicted to increase the rate of segregation. In order to test this prediction, we used two separate strategies for TFAM depletion to experimentally lower mtDNA copy in female germ cells during stages of oogenesis both before and after the post-natal bottleneck. TFAM is a critical factor in the replication, maintenance, and expression of $m t_{D N A}{ }^{125,145}$. Homozygous deletion of TFAM leads to the complete loss of mtDNA and is incompatible with post implantation development in the mouse, but heterozygotes are viable and exhibit a $\sim 50 \%$ reduction in $m t D N A$ in somatic tissues ${ }^{146}$. We found a similar reduction in copy number in PGCs isolated from TFAM+/- E10.5 embryos (Figure 6A) where the null allele was either maternally inherited or created by early-germline excision from a floxed allele, mediated by a Cre recombinase driven by the promoter for the gene encoding Tissue Non-specific Alkaline Phosphatase (TNAP-Cre) ${ }^{147}$, the original marker of PGCs ${ }^{49}$.

As predicted, the heteroplasmic distributions calculated from offspring born to heteroplasmic females from germlines derived from $\mathrm{TFAM}^{+/-}$PGCs exhibit greater variance (Figure S2) and a decrease in the estimated number of segregating units (about $50 \%$ of control) (Figure 5). In contrast, heterozygous deletion of TFAM by Zp3-Cre recombinase ${ }^{148}$ (activated in during late folliculogenesis and post-genetic bottleneck) ${ }^{149}$ did not alter rate of segregation 
(Figure S3) despite a near ten-fold reduction in mtDNA content of fertilized oocytes (Chapter 3: Figure ). The offspring of 6 independent lines of heteroplasmic TFAM ${ }^{\mathrm{fl} /+} ; \mathrm{Zp} 3-\mathrm{Cre}^{+}$females were analyzed $(\mathrm{n}=192)$.

We examined the primary oocytes of $\mathrm{TFAM}^{+/-}$ovaries from post-natal day (PND) 4 females by immunohistochemistry and confocal microscopy. We observed a qualitative and uniform decrease in TFAM immunofluorescence in primary oocytes as well as a decrease in the number of TFAM-positive punctae (which we interpret as mtDNA nucleoids) relative to control (Figure 7). Since TFAM is a required factor for all mtDNA molecules, the uniform decrease in TFAM protein (and presumably mtDNA copy number) did not, as we had predicted, alter size of the preferentially replicating population (Table 4).

Curiously, we observed significantly increased genotypic variances in offspring from recessive testcrosses between wild type males and TFAM ${ }^{+/-}$ heteroplasmic females $(p<0.0001)($ Table 4$)$, but not in the reciprocal testcross of TFAM $^{+/-}$male to wild type heteroplasmic female $(p=0.57)($ Figure 6B). When we examined embryos from these crosses, we discovered that PGCs isolated from $\mathrm{TFAM}^{+/-}$embryos that had inherited a deleted paternal TFAM allele had wild type levels of mtDNA (Figure 6A), but mature oocytes harvested from $\mathrm{TFAM}^{+-}$females generated by either type of recessive testcross had virtually indistinguishable levels of mtDNA (about $40 \%$ of control). We failed to detect a statistically significant increase in genotypic variance in E10.5 PGCs (Table S3) isolated from embryos that received either a maternally deleted TFAM allele or had excised a maternally inherited floxed allele through TNAP-Cre activity, which is activated in 
PGCs at $E 9^{147}$, even though mtDNA copy number was decreased by approximately 50\% (Figure 6A). However, by E11.5, we observed a significant increase in genotypic variance $(P=0.007125)$ (Figure $6 \mathrm{~A}$, Table 2$)$. These observations could be explained if further constriction of the physical bottleneck during early oogenesis by genetic depletion of maternal TFAM protein in the primordial germ cells were to prime a more rapid segregation of mtDNA at the postnatal genetic bottleneck. Therefore, we propose that the increased rate of segregation of mtDNA that is observed in offspring from TFAM heterozygous mothers is not a consequence of the relatively diminished number of replicating templates at the time of the genetic bottleneck (Figure 4B), but rather a function of an increased genotypic variance established during the migration of the primordial germ cells. 


\section{Analyzing the rate of segregation of human mtDNA sequence variants}

We decided to compare the rate of segregation of commonly inherited human point mutations in mtDNA to our heteroplasmic mouse model to determine whether the bottleneck size in humans and mice are similar.

Since the distribution of heteroplasmy in offspring is a function of the initial maternal allele frequency, it is imperative that single maternal lineages be used for comparisons of genotypic variances. Collecting a sufficiently large sample size for these analyses is a straightforward task when breeding animals or collecting germ cells from a developing murine ovary, but human pedigrees of comparable sizes, wherein mtDNA segregation can be investigated, are exceptionally rare ${ }^{83,150}$. To circumvent this problem, we adopted a strategy previously employed by Chinnery and coworkers ${ }^{75}$, where they analyzed recurrent mtDNA mutations by pooling a large number of human pedigrees. Rather than plotting the offspring's absolute heteroplasmic value versus the number of observations (which would have been uninformative due to the relatively small size of individual pedigrees), the offspring's heteroplasmic deviation (Mother's heteroplasmy value minus Offspring's heteroplasmy value), here denoted as $\mathrm{M}-\mathrm{O}$ value, is used, and plotted against the number of observations.

When we applied this transformation to the distributions of heteroplasmy from BALB/NZB heteroplasmic offspring the normality of these distributions was not significantly altered (data not shown). More importantly, we sought to test whether an $\mathrm{M}-\mathrm{O}$ transformation would affect the ability to detect significant 
differences in genotypic variance. We performed the same type of transformation on our two previously validated samples known to exhibit differences in genotypic variance: mouse PGCs and offspring from a single heteroplasmic maternal lineage. We use the group mean heteroplasmy value as the " $M$ " value and the individual heteroplasmy value of each cell as "O" value. The results of both nonparametric tests of equal variances demonstrate that the difference in genotypic variance that had been detected in the untransformed data $(p=0.002)$ still returned a statistically significant result with the transformed data $(p=0.002)$.

Our analyses of mtDNA mutations A3243G, T8993G, and G11778A showed no significant differences in transformed genotypic variances (Table S4), demonstrating that these mutations are all segregating at the similar rates. When we compared the segregation rates of these human mutations (represented by A3243G), to the segregation rates in wild type mice, we observed significant differences in genotypic variances indicating that the rate of segregation of mtDNA sequence variants in humans is significantly more rapid than in wild type mice ( $p=7.377 \mathrm{E}-09)$ but no different when compared to offspring from TFAM ${ }^{+/}$ females (Figure 8). 


\section{Discussion}

\section{The segregation of mtDNA sequence variants in the germline of the mouse}

We recently demonstrated that the segregation of mitochondrial DNA sequence variants in the female germline of the mouse is due the replication of a subgroup of templates in the post-natal ovary, which generates a distribution of heteroplasmy in the oocytes and offspring of the next generation that is predominantly shaped by random genetic drift. In this study, we estimated the size of the genetic bottleneck to be approximately 190 segregating units in our wild-type heteroplasmic mouse model, which segregates BALB and NZB mtDNAs. The population genetics equation originally employed in the calculation of bottleneck size used three parameters: the genotypic variance in the offspring, the maternal heteroplasmy level (which is defined as the mean of the offspring), and the number of generations required to produce a mature oocyte. Several assumptions were made to simplify the calculation of bottleneck size (that according to this equation would have resulted in an over-estimation by factor of 1.5), including setting the number of generations to a constant of 15 , which was done to reflect the number of mitotic divisions of the germline from primordial germ cell to mature oocyte. Implicit in this assumption was that all mtDNAs were undergoing equal and simultaneous replication throughout germline development. Having demonstrated the violation of this assumption, we realized that the number of generations (t) would have to vary with bottleneck size (Neff) if the number of segregating units (which we effectively equate to mitochondrial genomes) were to accurately reflect the empirically determined copy number in 
the mature oocyte/early embryo. Using this equation, we calculated the number of segregating units for a wild type heteroplasmic maternal lineages and found variability not only in the size of the bottleneck (Figure 5) but in the number of generations of mtDNA (Table 2) which we also observed in mice with a castaneus nuclear genetic background. Since our calculation of bottleneck size was based on a very large set of heteroplasmic offspring, we are confident that the variability we observe likely reflects real biology. We propose that the interlineage variability we observe in both the number of segregating units and the number of generations reflects the variable mtDNA copy number in the early embryo (Chapter 3, Figure 1) and in cells of the developing germline (Figure S1). It is worthwhile to note that even small changes in generation number can account for the variability in wild-type oocyte mtDNA content $(161,000 \pm 73,000$ copies). For example, if we take the offspring from WT 21 (Table 1), from which we calculate a bottleneck size of 230 units and 9.5 generations, the theoretical final copy number in the oocyte would be approximately 166,000 copies. If the number of replication events of these mtDNA templates were to vary by a single generation (either 8.5 or 10.5 generations) the mtDNA content would be between 83,000 and 333,000 copies. This range describes about $85 \%$ of our data and is easily accounted for by invoking the relaxed replication of mtDNA, which has been previously observed in somatic cells ${ }^{1}$ and has been proposed to occur during germline development ${ }^{151}$. It seems as though evolution has also allowed for some flexibility in mtDNA replication in the cells of the germline, although the 
consequences of venturing too far outside this biologically defined range may be detrimental. Such consequences will be discussed in the following chapter.

For the purposes of our calculations, we initially defined the segregating unit as a mitochondrial genome, although the nucleoid itself has been suggested to act as the segregating unit ${ }^{152}$. In vivo, mtDNA does not exist as a naked molecule of nucleic acid, but rather forms a protein-nucleic acid structure known as a nucleoid, in which several factors that also function in the transcription, replication, and maintenance effectively package mtDNA such that it can fit within the matrix compartment of the mitochondrion ${ }^{125}$. Studies performed in somatic cell culture systems estimate that nucleoids contain 2 to 10 copies of $m t D N A^{21,22}$. Morphological studies in mammalian primordial germ cells and oocytes suggest this number is closer to 1 or 2 genomes per nucleoid ${ }^{68,141}$, although a comprehensive study of nucleoid structure and content has yet to be performed in germ cells. In the calculation of bottleneck size, however, maintaining of a constant copy number per nucleoid throughout oogenesis is a far more important parameter than the actual number of copies of mtDNA per nucleoid in female germline.

Primary oocytes from $\mathrm{TFAM}^{+/}$animals have less TFAM protein, which results in about a two-fold decrease in mtDNA copy number and the number of segregating units. If the nucleoid were truly the segregating unit, we would expect a $\sim 50 \%$ reduction in the number of segregating units to result in a similar observed reduction in nucleoid number. Although it is difficult to precisely quantify nucleoid number in either wild-type of TFAM ${ }^{+/-}$ovarian sections (Figure 
5) given that the spatial resolution achieved by deconvolution is still insufficient to resolve all anti-TFAM immunofluorescent punctae (which we interpret as nucleoids), it is tempting to interpret the apparent concordance of these qualitative decreases (in mtDNA copy number, nucleoid number, bottleneck size) as evidence that the nucleoid is in fact the segregating unit.

While the segregation of mtDNA is expected to accelerate when the size of the bottleneck is reduced in the heterozygous TFAM female germline, we were surprised to discover that this was only the case when $\mathrm{TFAM}^{+-}$females inherited the null allele from the mother or when it was deleted by an early germline-acting Cre-recombinase (TNAP-Cre). Moreover, only maternal, but not paternal, inheritance of a null allele resulted in any significant decrease in mtDNA copy number in PGCs (Figure 6A). While there is no change in genotypic variance in the PGCs and oogonia of wild-type embryos, we observed that a further reduction in mtDNA copy number does significantly increase the genotypic variance in the migratory PGCs (by about E11.5). The genotypic variance of TFAM ${ }^{+-}$PGCs at E11.5 (but not yet at E10.5) exhibit normalized genotypic variance levels similar to those found in post-genetic bottleneck germ cells, which are lower than the genotypic variance observed in both $\mathrm{TFAM}^{+-}$mature oocytes and offspring (Figure 4). Taken together, these data suggest that the genotypic variance that may be produced following a further constriction of the physical bottleneck in $\mathrm{TFAM}^{+/}$germlines could further amplified by the post-natal mitochondrial genetic bottleneck. This as an attractive hypothesis and is consistent with the femalespecific segregation pattern observed in $\mathrm{TFAM}^{+/-}$oocytes and offspring, yet 
further measurements of mtDNA copy number and genotypic variance in $\mathrm{TFAM}^{+/-}$ embryonic germ cells sampled at and after gonad colonization are certainly warranted. Nevertheless, we propose that the biological range in mtDNA copy number in the PGCs represents the minimal pool of templates and possibly a threshold, below which rapid segregation of mtDNA would be expected to occur during oogenesis. Under wild-type conditions, most of genotypic variance that is observed in the mature oocytes and offspring of heteroplasmic females is in fact generated by the selection of a small subgroup of replicating genomes in the post-natal ovary, which is about $5 \%$ of the all the mtDNAs found in the primary oocyte of the primary follicle.

\section{The rate of segregation of human mtDNA sequence variants}

Heteroplasmic animal models, such as the ones used in this study, have provided a useful platform for the study of mtDNA segregation both in the germline $^{4,141}$ as well as in somatic tissues ${ }^{140,144}$. Besides the general advantages of animal models, the generally large numbers of offspring and litters provide sufficient sample sizes for the statistical comparisons of germline segregation patterns. We have generated a large set of heteroplasmic lineages to study the segregation of neutral sequence variants. The BALB and NZB sequence variants we use to track both the evolution of genotypic variance within a germline, as well as the distribution of heteroplasmy in offspring from separate maternal lineages, demonstrate that the selection of the small subgroup of mitochondrial genomes

that is chosen to repopulate the mature oocyte is entirely stochastic ${ }^{75,129,130}$. This 
is in stark contrast with the direction selection of severely deleterious mutations of mtDNA, which are rapidly eliminated over a few generations by a filter for purifying selection ${ }^{129,130}$. This process is likely activated during early oogenesis when the cellular content of mtDNA in the germline is low ${ }^{131,141}$. However, this filter does not eliminate all mutations, even those that cause nonsynonymous substitutions. Mutational analyses in mice and humans demonstrate that mutations in tRNA and rRNA genes, but not protein-coding genes, remain largely refractory from this selective elimination and while the functional basis for this discrepancy has yet to be uncovered, what is clear is that the transmission of tRNA mutations in humans (such as A3243G, G11778A, and A8344G), is, for the most part, stochastic ${ }^{75}$. Interestingly, tRNA genes comprise only $9 \%$ of the entire mitochondrial genome, but mutations in tRNA genes account for more than $60 \%$ of inherited mitochondrial DNA diseases. We observed that the rate of segregation of the most common mutations causing MELAS (A3243G), LHON (G11778A), and even NARP (which is caused by a mutation at position 8993 , in the ATP6 gene), segregate at similar rates. So in this study A3243G, T8993G, and $\mathrm{A} 11778 \mathrm{G}$ are used as neutral markers in order to compare the rate of segregation of human and mouse mtDNA sequence variants in the absence of a truly reliable and well-documented categorically neutral and stable marker. We observe that the rates of segregation of these human mutations is equivalent to that of BALB and NZB mtDNA segregating in the germline of $\mathrm{TFAM}^{+/-}$females, but is much more rapid than in wild-type mice. This species-specific discrepancy points to a difference in the size of the genetic bottleneck. If the mean number of 
mtDNAs present in the mature oocytes of both humans and mice are similar, the narrow human genetic bottleneck could be related to the degree of constriction at the physical bottleneck or the number of generations of mtDNA. Electron micrographs of human PGCs reveal far fewer mitochondria than what has been reported in mouse PGCs by immunocytochemistry, although it should be noted that the accurate calculation of mitochondrial number from ultrathin sections does not provide as accurate an estimate as does the 3D reconstruction of an entire mouse PGC from Z-slices acquired by confocal microscopy ${ }^{6}$. Still, if the mitochondrial number were lower in human PGCs, more mitochondrial DNA replication events would be required to repopulate the oocyte, which by itself could increase the genotypic variance. However, if the amplification of mtDNA in the germline were in fact starting from a relatively lower mtDNA copy number, as is the case in the germline of the $\mathrm{TFAM}^{+/-}$female embryos, it is possible that a substantial amount of genotypic variance would be generated during embryogenesis, which would be further amplified by the mitochondrial genetic bottleneck.

An alternative explanation is that the rate of segregation of these human mutations is in fact more rapid than neutral human polymorphisms in mtDNA. While random genetic drift is clearly responsible for the distribution of heteroplasmy of these mutations, perhaps there is some yet-unidentified mechanism that specifically accelerates the rate of segregation of pathogenic mutations that have escaped the filter for purifying selection (such as those mutations that cause MERRF, MELAS, and LHON). While it is difficult for us to 
imagine the functional basis of such a mechanism of selection, particularly since low mutant loads of these mutations rarely elicit a biochemical deficiency at the cellular or organismal level, this explanation will remain a formal possibility until such time as stable and neutral markers of human mtDNA are identified and employed in large-scale segregation studies. 


\section{Materials and Methods}

\section{Transgenic and Congenic Mice}

All experiments were conducted according to established ethical guidelines for the care and use of laboratory animals. The McGill University Animal Care Committee approved all experimental protocols. Heteroplasmic floxed TFAM mice were generated by crossing previously described OG2-BALB/NZB females $^{141}$ to $\operatorname{TFAM}^{\mathrm{f} / \mathrm{fl}}$ males $^{146}$. F1 females were then backcrossed to TFAM ${ }^{\mathrm{f} / \mathrm{fl}}$ males for 5 generations to generate heteroplasmic floxed TFAM females that also carried the GOF18deltaPE transgene, henceforth termed TFAM ${ }^{\mathrm{ft} / \mathrm{I}} \mathrm{OG} 2-$ BALB/NZB. To generate heterozygous germline $\operatorname{TFAM}^{+/-} \mathrm{OG} 2-\mathrm{BALB} / \mathrm{NZB}$ animals, we crossed males carrying the early acting TNAP-Cre recombinase transgene ${ }^{147}$ or the late-acting $Z p 3-C r e$ recombinase transgene ${ }^{148}$ to TFAM ${ }^{f / f l}$ OG2-BALB/NZB females. Genotyping for the presence of the GOF18deltaPE transgene ${ }^{141}$, the TNAP-Cre recombinase transgene ${ }^{147}$, the Zp3Cre recombinase transgene ${ }^{148}$ were performed as previously described. We used previously described methods for TFAM genotyping of offspring ${ }^{153}$.

Isolation of cleavage stage embryos, oocytes, single embryonic germ cells. Embryos and germ cells were isolated as previously described ${ }^{141}$.

\section{Mitochondrial DNA Copy number and Heteroplasmy Quantification}

Mitochondrial DNA heteroplasmy and copy number measurements were determined as previously described ${ }^{141}$. 


\section{Confocal Microscopy and Immunohistochemistry}

Confocal microscopy and immunohistochemistry for TFAM detection in ovarian sections was performed as previously described ${ }^{141}$.

\section{Statistical Testing}

Chi-squared tests and non-parametric tests of equal variances (Levene and Bootstrapped Levene tests with the Brown-Forsythe correction) were performed as previously described. Pairwise t-tests were performed to test for equal means between groups. 


\section{Figure Legends}

\section{Figure 1: Heteroplasmy Distributions of offspring from mothers with}

varying levels of NZB mtDNA.

Frequency histograms of \%NZB heteroplasmy levels measured in offspring from different maternal lineages. Maternal heteroplasmy levels vary from $10 \%$ to $80 \%$ and are segregating on M.m.domesticus nuclear genetic backgrounds unless otherwise denoted as CAST (M.m.castaneus).

Figure 2: Normalization of Genotypic Variance in the Calculation of

\section{Bottleneck Size}

Normalized variance was calculated from the variance measured in female germ cells and offspring using the equation $\mathrm{V} / \mathrm{p} 0(1-\mathrm{p} 0)$, where $\mathrm{V}$ is the genotypic variance and $\mathrm{p} 0$ is the mean heteroplasmy level of the sample (i.e. maternal mean heteroplasmy level). This transformation neutralizes the relationship (A) between genotypic variance (V) and initial heteroplasmy level (p0) (B). So the absence of a relationship between bottleneck size and maternal heteroplasmy level (C) indicates that the normalized variance used in the calculation of bottleneck size represents a useful way of transforming the data to remove bias. 
Figure 3: Tracking the evolution of normalized genotypic variance in female gametogenesis.

By plotting the normalized genotypic variance as a function of either days postcoitum (dpc) or post-natal days (PND), we are able to track the evolution of genotypic variance (A). We observe no statistically significant changes in normalized variance during pre-natal gametogenesis. There is an increase in normalized variance during post-natal oocyte maturation (B) that is reflected in offspring (C). In TFAM ${ }^{+/-}$mature oocytes and offspring have exhibit increased normalized genotypic variance.

Figure 4: The proportion of replicating templates declines during germline development

The segregating units of mtDNA expressed as a proportion of the number of copies of mtDNA declines as the germline develops. This proportion declines precipitously in post-natal oocytes. The proportion of replicating templates remains unchanged in $\mathrm{TFAM}^{+-}$oocytes and offspring despite a decrease in mtDNA copy number. The proportion of replicating genomes drops below $5 \%$ at the mitochondrial genetic bottleneck and remains below $1 \%$ for the remainder of folliculogenesis. 
Figure 5: Calculating the Size of the Mitochondrial Genetic Bottleneck in Wild Type and TFAM Heterozygous Maternal Lineages

The effective number of segregating units was calculated for the mitochondrial DNA segregating on both M.m.domesticus (grey) and M.m.castaneus (white) as well as for the heteroplasmic offspring born to TFAM heterozygous mothers (black) using the Sewell Wright-derived equation where $t$ varies as a function of the number of segregating units required to generate a given normalized genotypic variance and mtDNA content in the mature oocyte. The effective number of segregating units was calculated based on the heteroplasmic offspring segregating BALB and NZB mtDNA on either a mixed domesticus genetic background (WT), $\mathrm{TFAM}^{+/-}$genetic background, M.m.domesticus (grey), or M.m.castaneus (white) nuclear genetic background. The effective number of segregating units in the germlines of $\mathrm{TFAM}^{+/-}$females has a mean value of 85 , which is roughly half that of wild type females and significantly different (Student's t-test; $p=2.85 E-05)$. Mean bottleneck sizes for pure domesticus (BALB) and castaneus (CAST), which were 150 and 135 segregating units, respectively, did not differ significantly (Student's t-test $p=0.6$ ). The mean bottleneck size for WT females was 189 segregating units, which was not significantly different from the pure domesticus bottleneck size $(\mathrm{p}=0.2)$. 
Figure 6: Maternal, but not paternal germline deletion of TFAM affects mtDNA copy number and segregation rate.

(A) Primordial germ cells (PGCs) isolated from E10.5 $\mathrm{TFAM}^{+/-}$female embryos (white) contain 1333 copies of mtDNA while those from WT E10.5 female embryos (grey) contain 2764 copies. E11.5 female TFAM ${ }^{+-}$PGCs (black) contain 437 copies of mtDNA. E9.5 TFAM ${ }^{+/}$female PGCs isolated from embryos where the null allele was paternally inherited contain normal levels of mtDNA (2126 copies). TFAM null alleles were created by the TNAP-Cre excision of a floxed TFAM allele at E9 or by maternal inheritance resulted in similar decreases in germ cells isolated after E10.5. (B) Increased genotypic variance is observed in maternal (grey) but not paternal (black) recessive testcrosses of $\mathrm{TFAM}^{+/}$animals.

\section{Figure 7: Primary Oocytes in TFAM ${ }^{+-}$Ovaries Contain less TFAM Protein and Fewer Nucleoids}

Primary oocytes from TFAM ${ }^{+/-}$PND 4 females exhibit a qualitative decrease in TFAM immunofluorescence compared to WT PND 4 primary oocytes processed simultaneously, imaged using the same settings by confocal microscopy, and deconvolved using the same empirical PSF and number of Z-slices. While the subcellular distribution of nucleoids is unchanged, $\mathrm{TFAM}^{+/-}$primary oocytes, contain fewer nucleoids. 
Figure 8: Mitochondrial DNA segregation rates differ between mice and humans

Frequency histograms depict the distribution of mother-offspring (M-O)

transformations used to illustrate the rate of segregation of pooled data. Inherited human mtDNA mutations, such as the A3243G mutation that causes MELAS (A), segregate more rapidly than BALB and NZB mtDNA in wild type mouse germlines (C), but just as rapidly as in TFAM+/- germlines (B).

Table 1: Calculations of bottleneck size and generation number from groups of cells from the developing female germline Normalized variance, generation number ( $\mathrm{t}$ ), bottleneck size (Neff), and the proportion of replicating templates were calculated from the group mean heteroplasmy level p0) and variance (V) measured in cells sampled during female germline development.

Table 2: Calculations of bottleneck size and generation number from wildtype offspring

Normalized variance, generation number ( $t$ ), bottleneck size (Neff), and the proportion of replicating templates were calculated from the group mean heteroplasmy level p0) and variance (V) measured in tail biopsies sampled from wild-type offspring at 3 weeks of life. 
Table 3: Calculations of bottleneck size and generation number from domesticus and castaneus offspring Normalized variance, generation number ( $t$ ), bottleneck size (Neff), and the proportion of replicating templates were calculated from the group mean heteroplasmy level p0) and variance (V) measured in tail biopsies sampled from domesticus (BALB) or castaneus (CAST) offspring at 3 weeks of life.

Table 4: Calculations of bottleneck size and generation number from offspring from $\mathrm{TFAM}^{+/-}$mothers or parents.

Normalized variance, generation number ( $t$ ), bottleneck size (Neff), and the proportion of replicating templates were calculated from the group mean heteroplasmy level p0) and variance (V) measured in tail biopsies sampled offspring from TFAM ${ }^{+/-}$mothers or parents at 3 weeks of life.

Figure S1: Biological variability in mtDNA copy number in the female germline

Frequency histograms of mtDNA copy number measured in female germ cells sampled at $9.5(A), 10.5(B), 12.5(C)$, and $14.5(D)$ days post-coitum illustrate the biological variability of copy number in the germline. 
Figure S2: Late germline excision of a null TFAM allele does not alter the genotypic variance in offspring of the next generation.

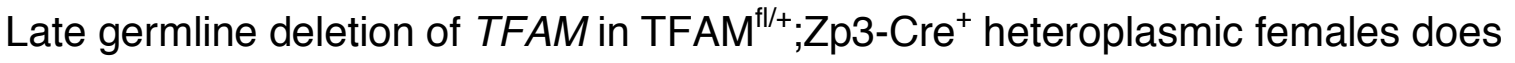
not result in an increased genotypic variance in offspring (A-F). Distributions of heteroplasmy in $\mathrm{TFAM}^{+/-}$(black) and wild-type littermates are not statistically different according to non-parametric test of equal variances.

Figure S3: Early germline excision, or maternal inheritance, of a null TFAM allele increases the genotypic variance in offspring of the next generation. Maternal inheritance of a deleted TFAM allele (A-D) or early germline excision of a floxed TFAM allele by TNAP-Cre (E) increases the genotypic variance calculated from total offspring in the next generation. The heteroplasmic variance of TFAM ${ }^{+/-}$offspring born to a TFAM ${ }^{\mathrm{fl} /} ; \mathrm{TNAP}^{-} \mathrm{Cre}^{+}$mothers is larger than the variance measured in wild-type littermates $(F)$.

Table S1: Inter-litter pairwise comparisons of genotypic variance in offspring from domesticus and castaneus females.

Levene (horizontal) and Bootstrapped Levene (vertical) tests of equal variances with the Brown-Forsythe correction were applied to the genotypic variances measured in litters from heteroplasmic females segregating BALB and NZB mtDNA on either domesticus or castaneus nuclear genetic backgrounds. Red fills indicate statistically significant differences in genotypic variance $(p>0.5)$. 
Table S2: Pairwise comparisons of genotypic variances in offspring either domesticus or castaneus heteroplasmic offspring.

Levene (horizontal) and Bootstrapped Levene (vertical) tests of equal variances with the Brown-Forsythe correction were applied to the genotypic variances measured in total offspring from heteroplasmic females segregating BALB and NZB mtDNA on either domesticus (A) or castaneus $(\mathrm{B})$ nuclear genetic backgrounds. Red fills indicate statistically significant differences in genotypic variance $(p>0.5)$.

Table S3: Pairwise comparisons of genotypic variance in migratory PGCs from $\mathrm{TFAM}^{+/}$female germlines.

The Levene test $(A)$ and Bootstrapped Levene $(B)$ tests of equal variances with the Brown-Forsythe correction were used to compare the genotypic variances measured in PGCs from TFAM ${ }^{+/-}$female embryos isolated at E10.5 and E11.5. While, E10.5 TFAM+/- female PGCs contained 1333 copies of mtDNA, roughly half that of WT E10.5 PGCs, we fail to detect a significant increase in genotypic variance at this stage. However, PGCs from $\mathrm{TFAM}^{+/-}$female embryos isolated at E11.5 exhibit a genotypic variance equivalent to that measured in post-natal oocytes that have already passed through the mitochondrial genetic bottleneck. Red fills denote $p>0.01$ and Yellow fills denote $p>0.05$. 


\section{Table S4: Segregation Pattern of Inherited Human Pathogenic Mutations}

The rates of segregation of heteroplasmic pathogenic human mutations (A3243G, T8993C, and A11778G) were compared using the Levene (horizontal) and Bootstrapped Levene Test for equal variances with the Brown-Forsythe correction. Published pedigrees with clear and quantitated heteroplasmic segregation were transformed before pooling (see Methods). In the case of the A3243G mutation, the transformed and pooled data reflect the mutant load measured in blood, hair, or urine. Probands were excluded, although inclusion in the transformed and pooled data did not affect the statistical significance of the result. Red fills denote $p>0.01$ and Yellow fills denote $p>0.05$. 
Figure 1





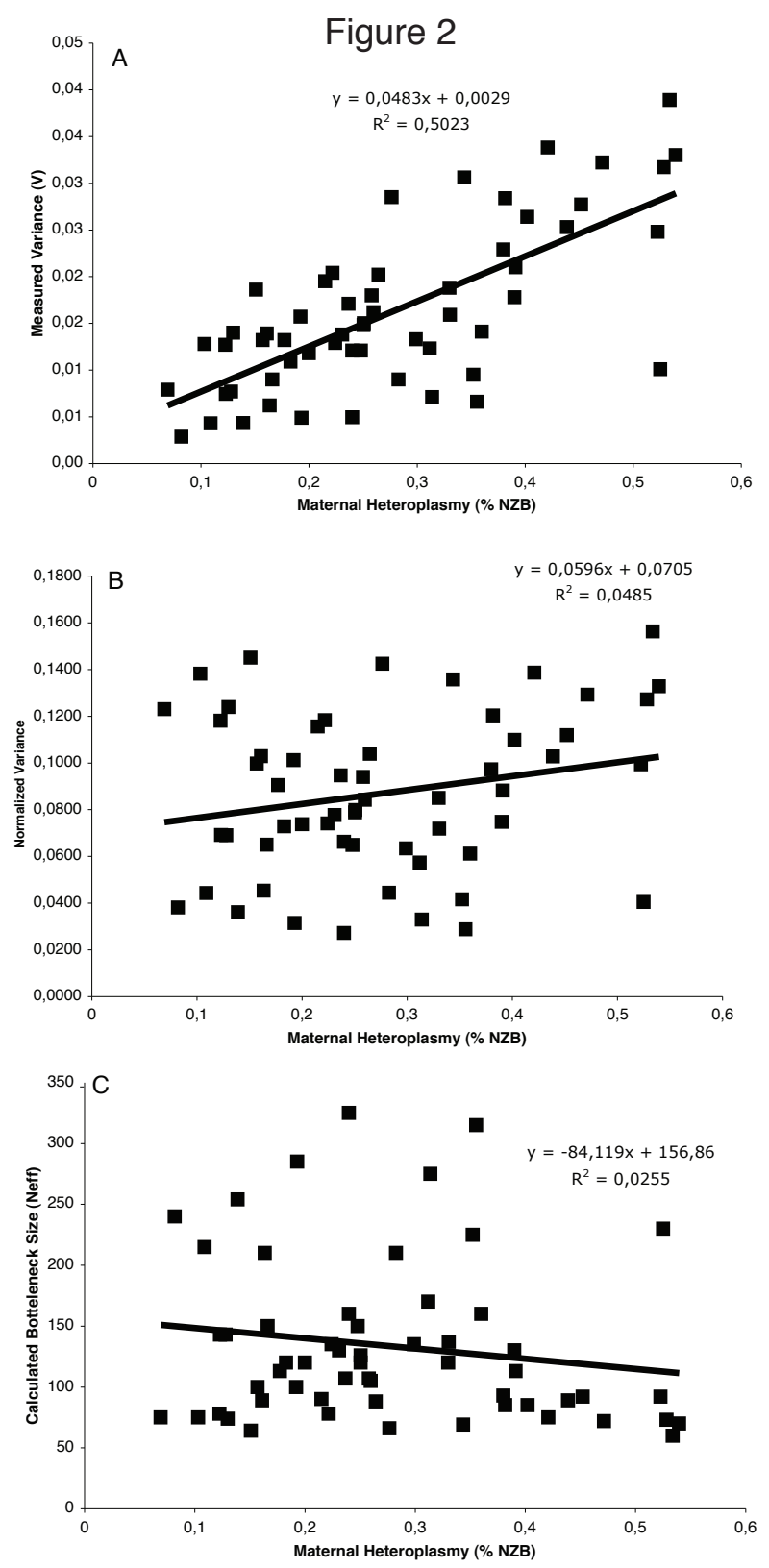
Figure 3
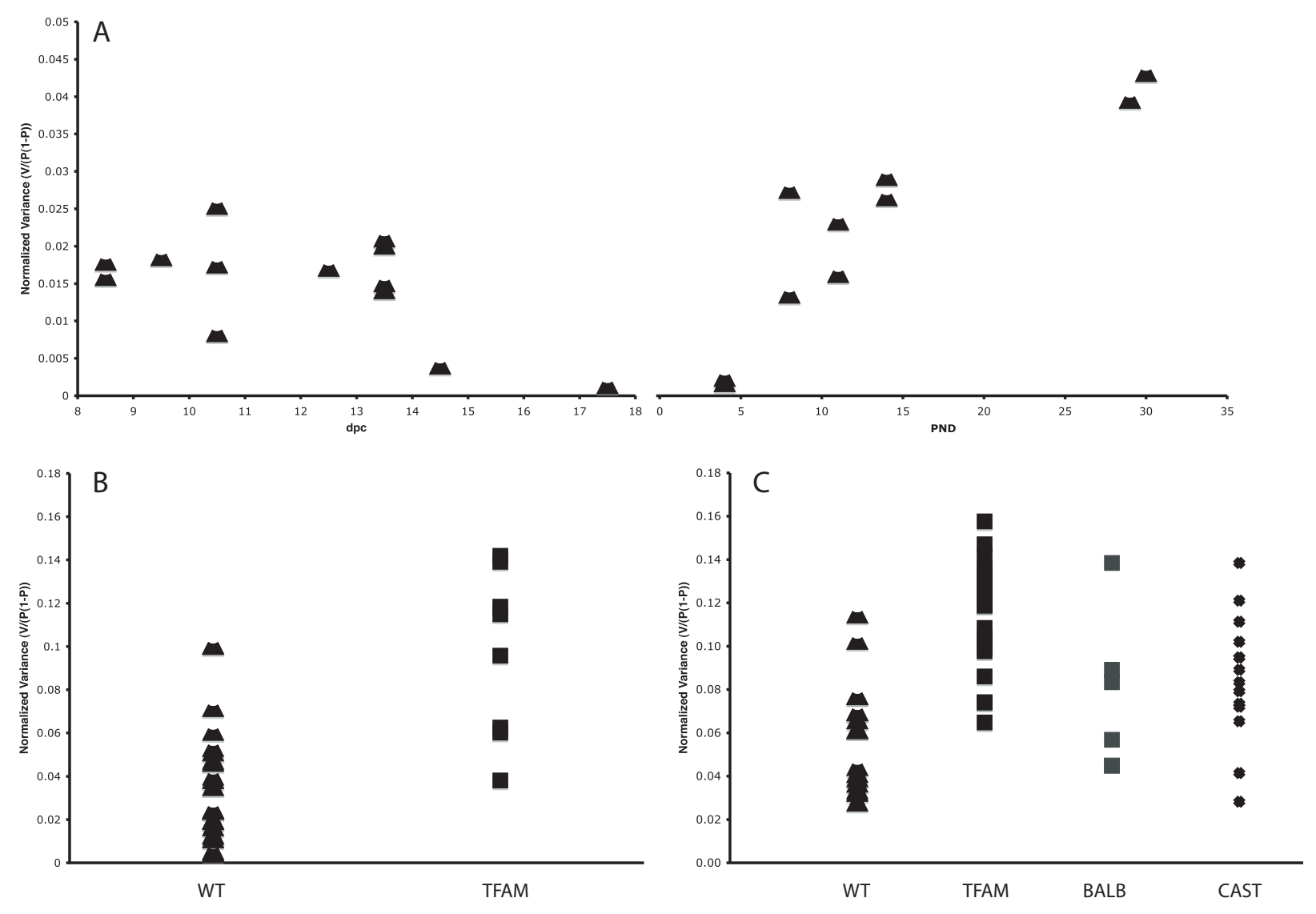


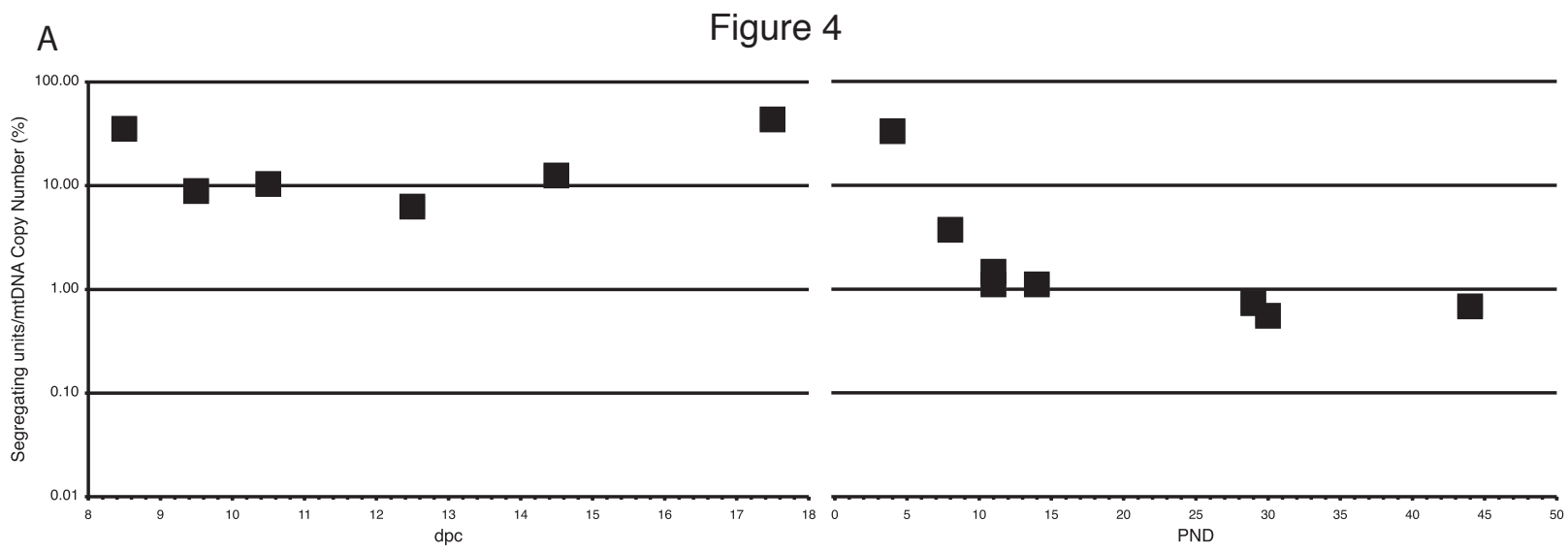

B

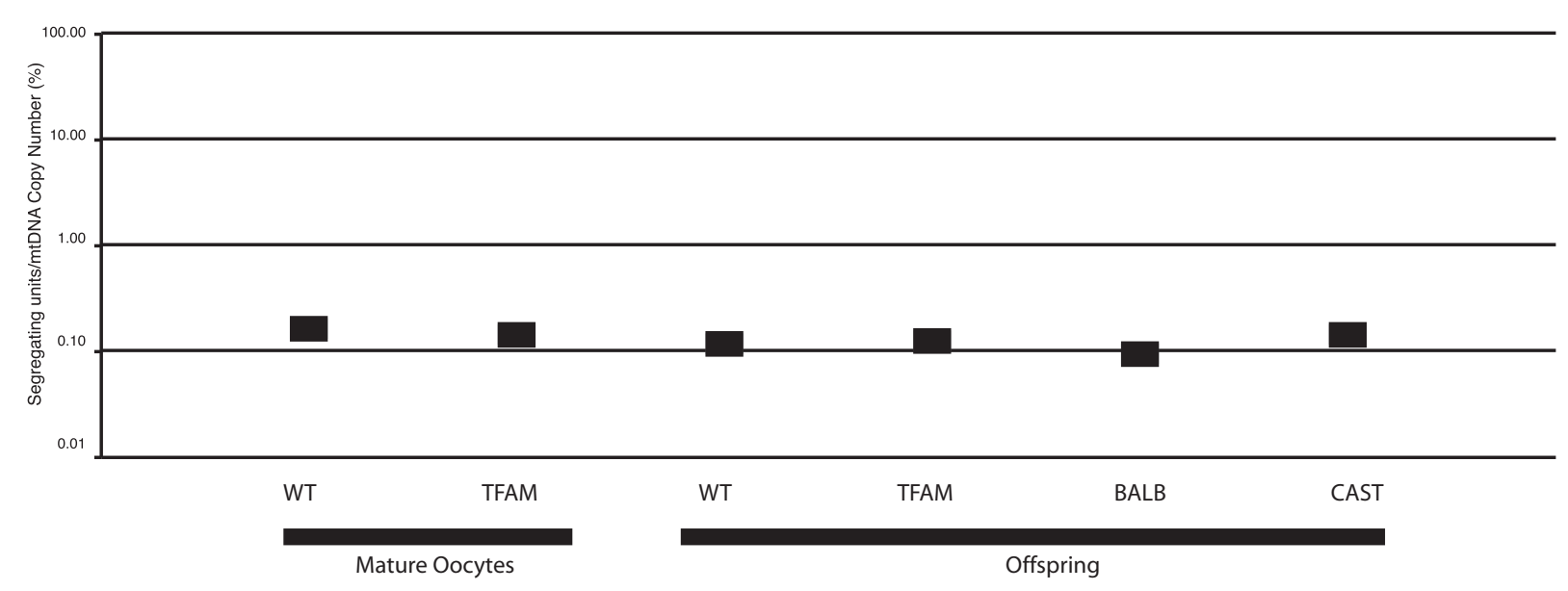


Figure 5

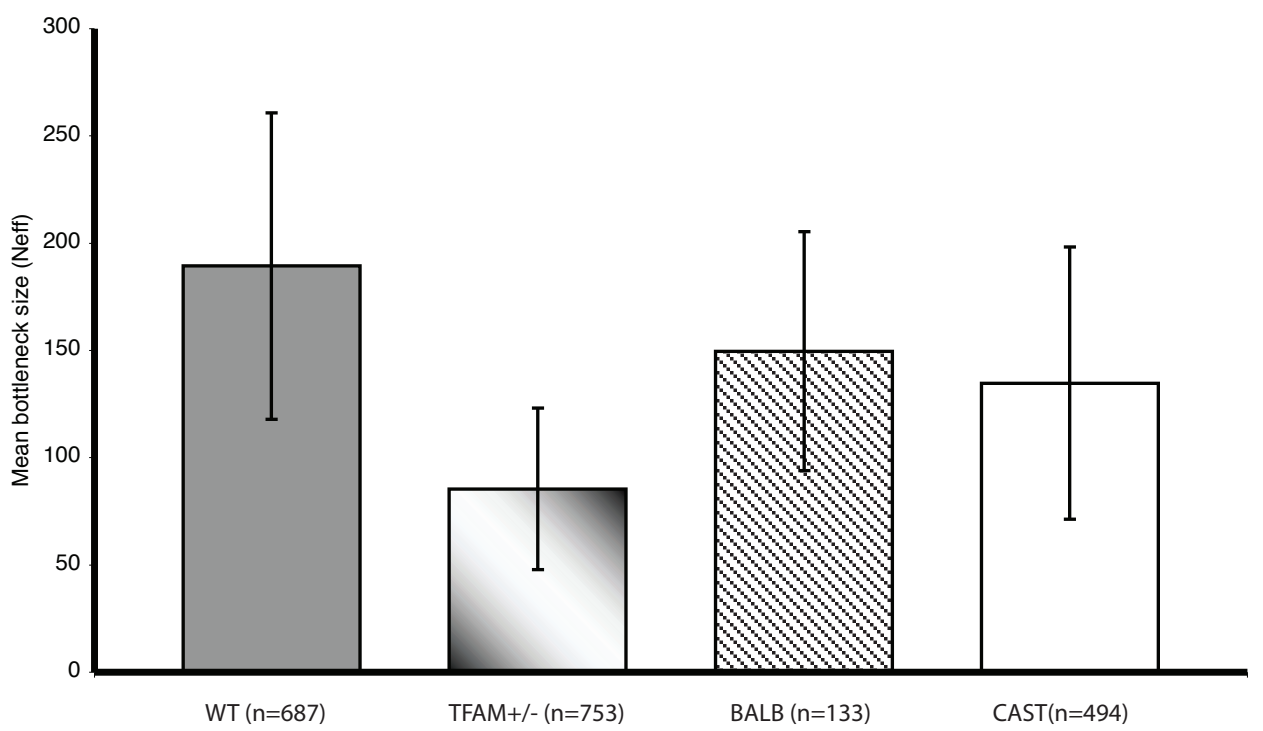


Figure 6

Figure 7
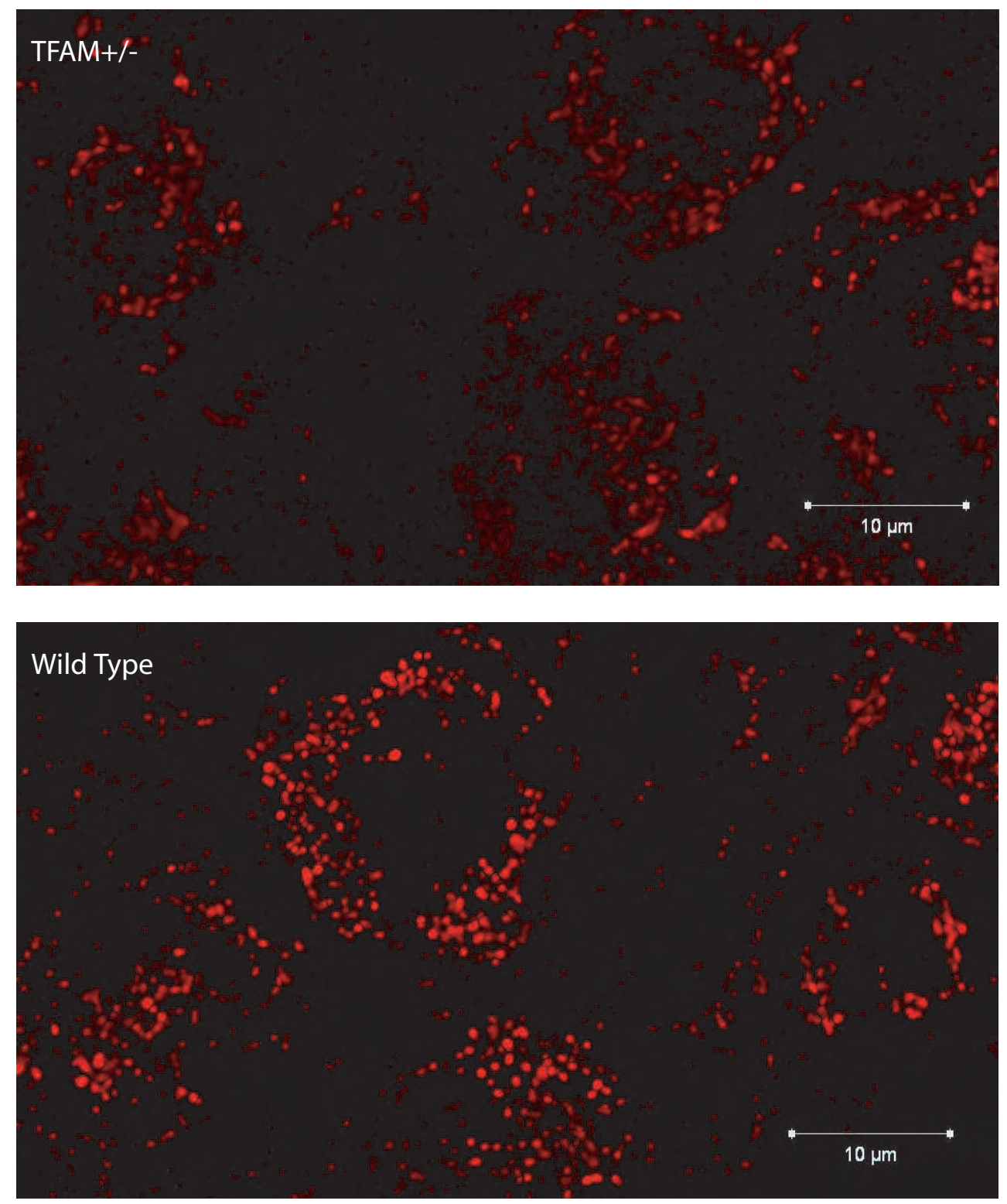
Figure 8
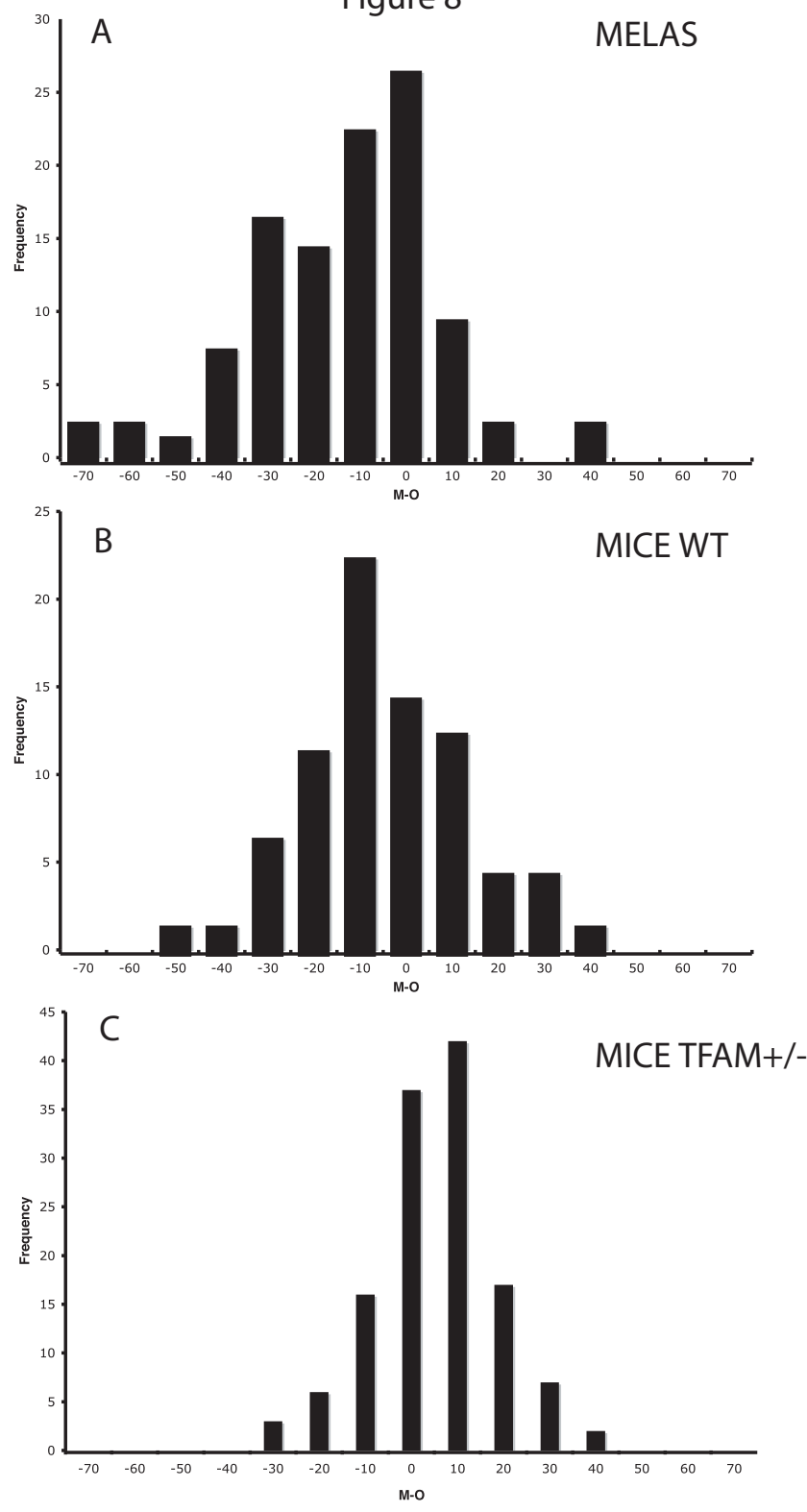
Table 1

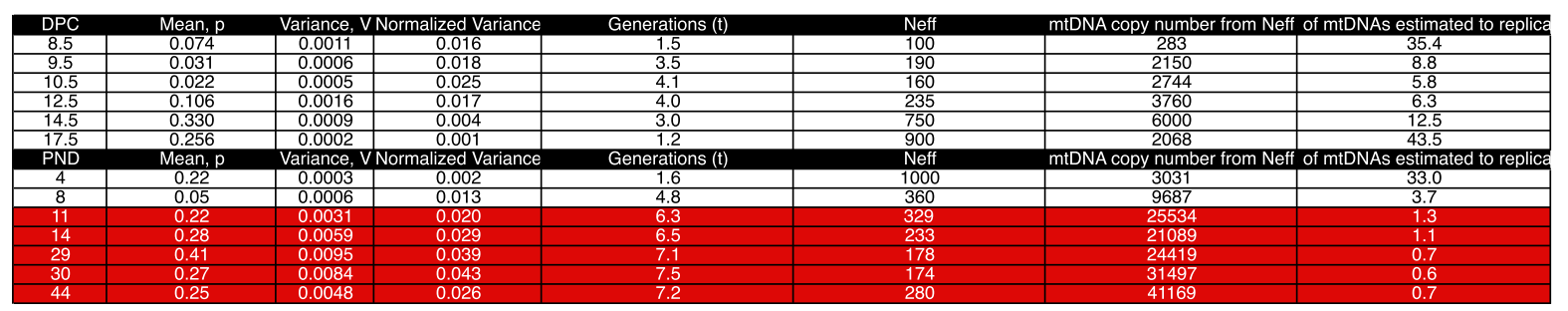


Table 2

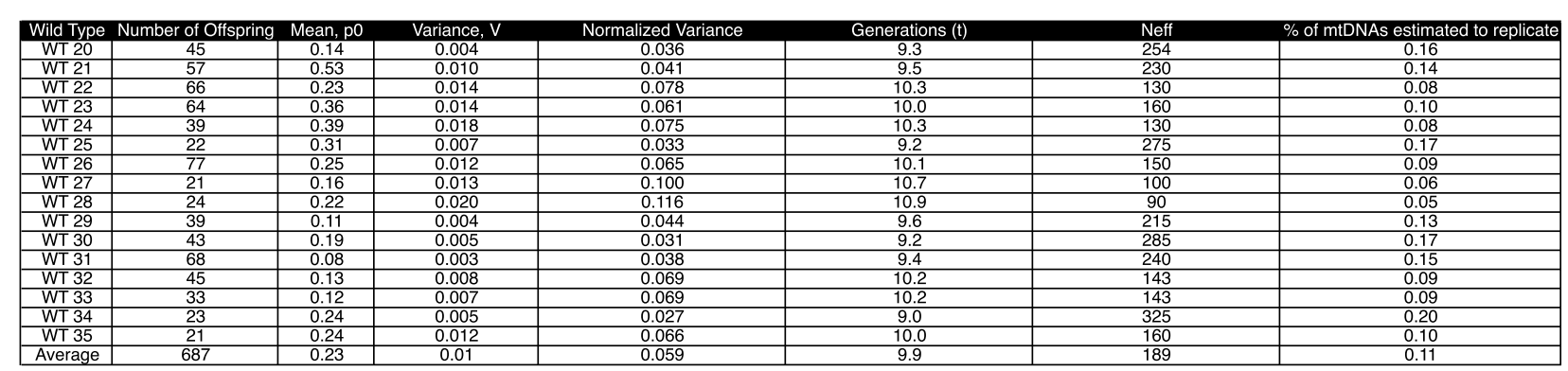


Table 3

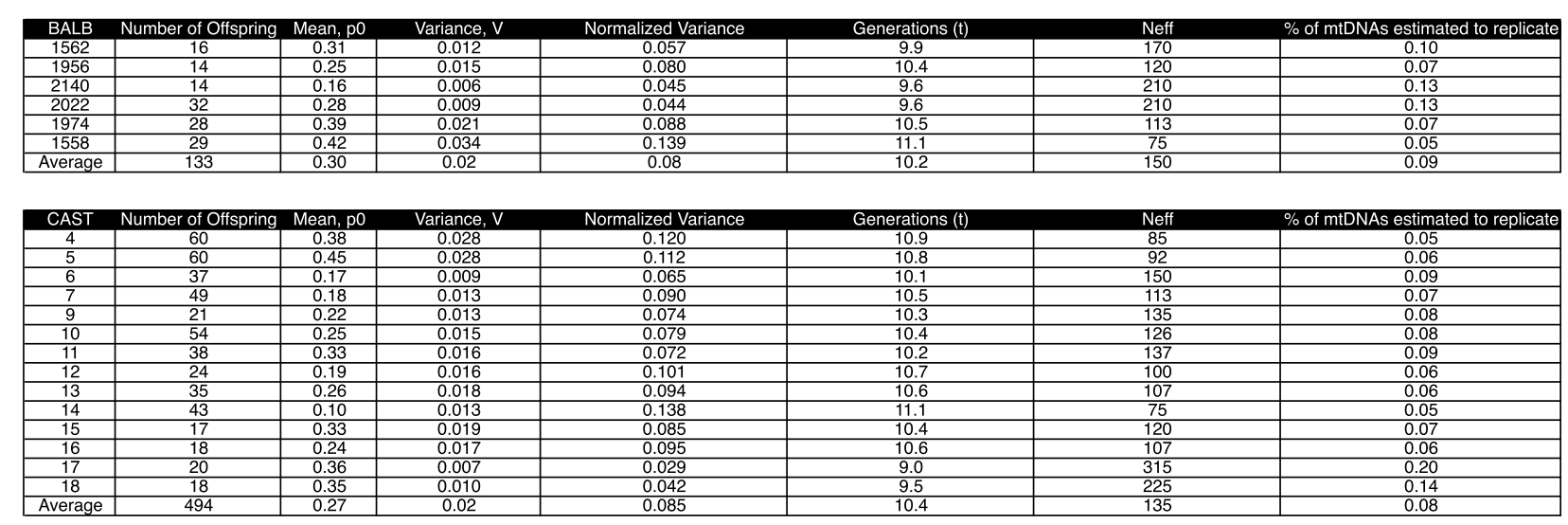


Table 4

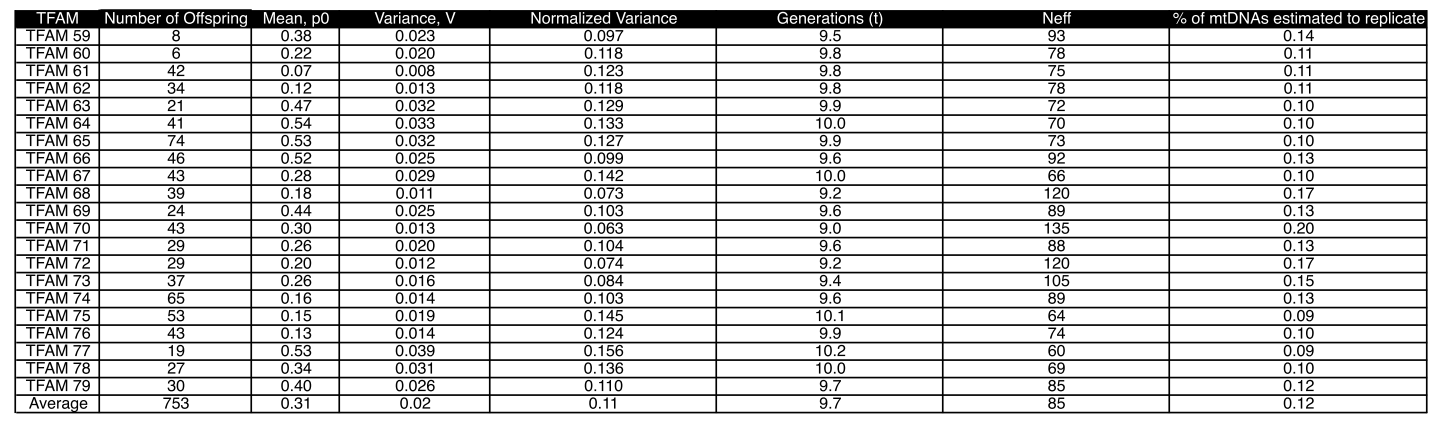


Figure S1
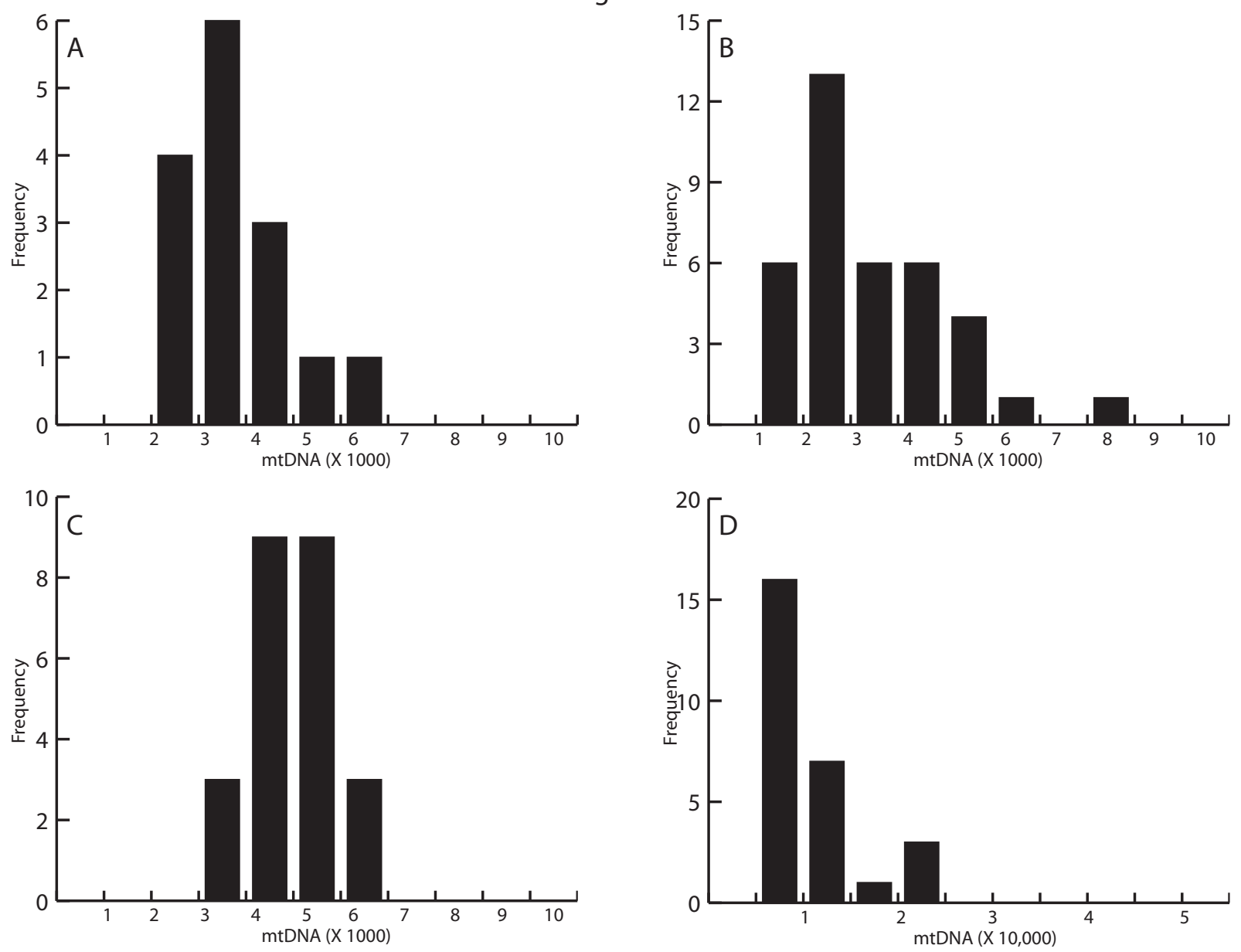
Figure S2
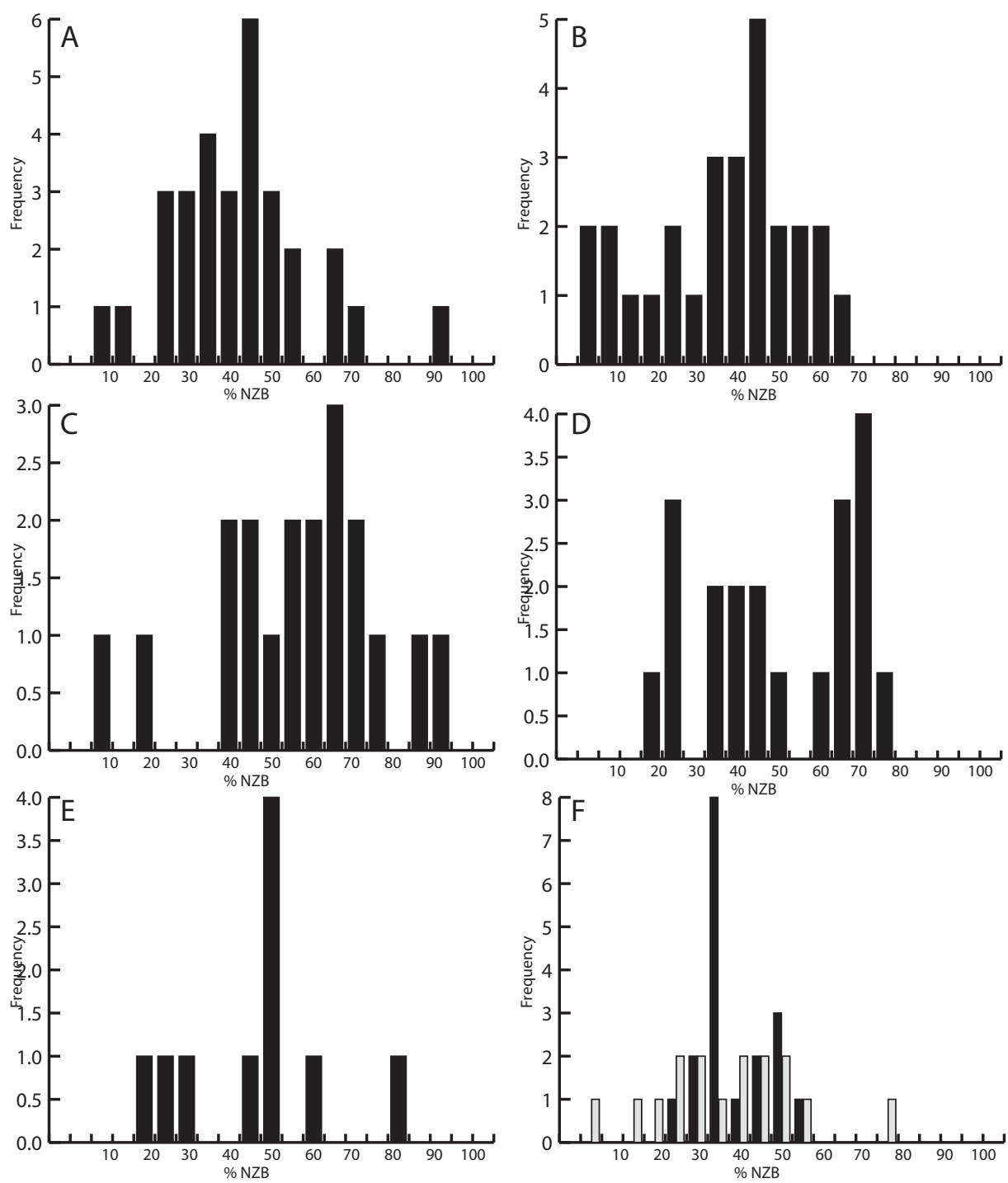
Figure $\mathrm{S} 3$

Table S1

\begin{tabular}{|c|c|c|c|c|}
\hline BALB 1562 & litter1 & litter2 & litter3 & litter4 \\
\hline litter1 & & 0.755 & 0.365 & 0.92 \\
\hline litter2 & 0.755 & & 0.516 & 0.693 \\
\hline litter3 & 0.365 & 0.516 & & 0.347 \\
\hline litter4 & 0.92 & 0.693 & 0.347 & \\
\hline BALB 1956 & litter1 & litter2 & litter3 & litter4 \\
\hline litter1 & & 0.159 & 0.343 & 0.35 \\
\hline litter2 & 0.159 & & 0.914 & 0.108 \\
\hline litter3 & 0.343 & 0.914 & & 0.542 \\
\hline litter4 & 0.35 & 0.108 & 0.542 & \\
\hline
\end{tabular}

\begin{tabular}{|c|c|c|c|}
\hline BALB 2140 & litter1 & litter2 & litter3 \\
\hline litter1 & & 0.071 & 0.876 \\
\hline litter2 & 0.071 & & 0.223 \\
\hline litter3 & 0.876 & 0.223 & \\
\cline { 1 - 2 } & &
\end{tabular}

\begin{tabular}{|c|c|c|c|c|c|c|c|c|c|}
\hline BALB 2022 & litter1 & litter2 & litter3 & litter4 & litter5 & litter6 & litter7 & litter8 & litter9 \\
\hline litter1 & & 0.165 & 0.34 & 0.857 & & 0.826 & 0.89 & 0.985 & 0.572 \\
\hline litter2 & 0.165 & & 0.585 & 0.099 & 0.076 & 0.07 & 0.24 & 0.151 & 0.085 \\
\hline litter3 & 0.34 & 0.585 & & 0.245 & 0.169 & 0.183 & 0.553 & 0.462 & 0.519 \\
\hline litter4 & 0.857 & 0.099 & 0.245 & & 0.835 & 0.973 & 0.58 & 0.681 & 0.248 \\
\hline litter5 & & 0.076 & 0.169 & 0.835 & & 0.808 & 0.526 & 0.643 & 0.142 \\
\hline litter6 & 0.826 & 0.07 & 0.183 & 0.973 & 0.808 & & 0.509 & 0.652 & 0.205 \\
\hline litter7 & 0.89 & 0.24 & 0.553 & 0.58 & 0.526 & 0.509 & & 0.853 & 0.747 \\
\hline litter8 & 0.985 & 0.151 & 0.462 & 0.681 & 0.643 & 0.652 & 0.853 & & 0.584 \\
\hline litter9 & 0.572 & 0.085 & 0.519 & 0.248 & 0.142 & 0.205 & 0.747 & 0.584 & \\
\hline
\end{tabular}

\begin{tabular}{|c|c|c|c|}
\hline BALB 2166 & litter1 & litter2 & litter3 \\
\hline litter1 & & 0.579 & 0.206 \\
\hline litter2 & 0.579 & & 0.103 \\
\hline litter3 & 0.206 & 0.103 & \\
\hline
\end{tabular}

\begin{tabular}{|c|c|c|c|c|c|c|c|c|}
\hline BALB 1974 & litter1 & litter2 & litter3 & litter4 & litter5 & litter6 & litter7 & litter8 \\
\hline litter1 & & 0.795 & 0.204 & 0.617 & 0.449 & 0.072 & 0.387 & 0.061 \\
\hline litter2 & 0.795 & & 0.086 & 0.953 & & & 0.859 & 0.484 \\
\hline litter3 & 0.204 & 0.086 & & 0.385 & 0.089 & 0.084 & 0.207 & 0.037 \\
\hline litter4 & 0.617 & 0.953 & 0.385 & & 0.988 & 0.427 & 0.789 & 0.369 \\
\hline litter5 & 0.449 & & 0.089 & 0.988 & & & 0.897 & 0.559 \\
\hline litter6 & 0.072 & & 0.084 & 0.427 & & & 0.417 & 0.265 \\
\hline litter7 & 0.387 & 0.859 & 0.207 & 0.789 & 0.897 & 0.417 & & 0.504 \\
\hline litter8 & 0.061 & 0.484 & 0.037 & 0.369 & 0.559 & 0.265 & 0.504 & \\
\hline
\end{tabular}

\begin{tabular}{|c|c|c|c|c|c|c|c|c|}
\hline BALB 1558 & litter1 & litter2 & litter3 & litter4 & litter5 & litter6 & litter8 & litter9 \\
\hline litter1 & & 0.461 & 0.354 & 0.139 & 0.734 & 0.409 & 0.329 & \\
\hline litter2 & 0.461 & & 0.454 & 0.777 & 0.476 & 0.872 & 0.278 & 0.341 \\
\hline litter3 & 0.354 & 0.454 & & 0.184 & 0.902 & 0.404 & 0.128 & 0.175 \\
\hline litter4 & 0.139 & 0.777 & 0.184 & & 0.188 & 0.983 & 0.181 & 0.082 \\
\hline litter5 & 0.734 & 0.476 & 0.902 & 0.188 & & 0.359 & 0.131 & 0.366 \\
\hline litter6 & 0.409 & 0.872 & 0.404 & 0.983 & 0.359 & & 0.332 & 0.372 \\
\hline litter8 & 0.329 & 0.278 & 0.128 & 0.181 & 0.131 & 0.332 & & 0.246 \\
\hline litter9 & & 0.341 & 0.175 & 0.082 & 0.366 & 0.372 & 0.246 & \\
\hline
\end{tabular}

\begin{tabular}{|c|c|c|c|c|}
\hline CAST 03 & litter1 & litter2 & litter3 & litter4 \\
\hline litter1 & & 0.481 & 0.098 & 0.196 \\
\hline litter2 & 0.481 & & 0.032 & 0.051 \\
\hline litter3 & 0.098 & 0.032 & & 0.059 \\
\hline litter4 & 0.196 & 0.051 & 0.059 & \\
\hline
\end{tabular}

\begin{tabular}{|c|c|c|c|c|c|}
\hline CAST 06 & litter1 & litter2 & litter3 & litter4 & litter5 \\
\hline litter1 & & 0.837 & 0.206 & 0.191 & 0.022 \\
\hline litter2 & 0.837 & & 0.187 & 0.174 & 0.018 \\
\hline litter3 & 0.206 & 0.187 & & 0.419 & 0.776 \\
\hline litter4 & 0.191 & 0.174 & 0.419 & & 0.096 \\
\hline litter5 & 0.022 & 0.018 & 0.776 & 0.096 & \\
\hline
\end{tabular}


Table S2

Pairwise $\mathrm{p}$ values for Tests for Equal Variances (Brown-Forsythe Corrected) Levene Test

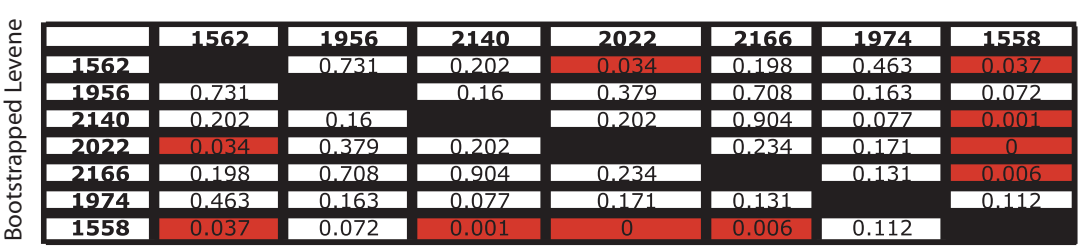

Levene Test

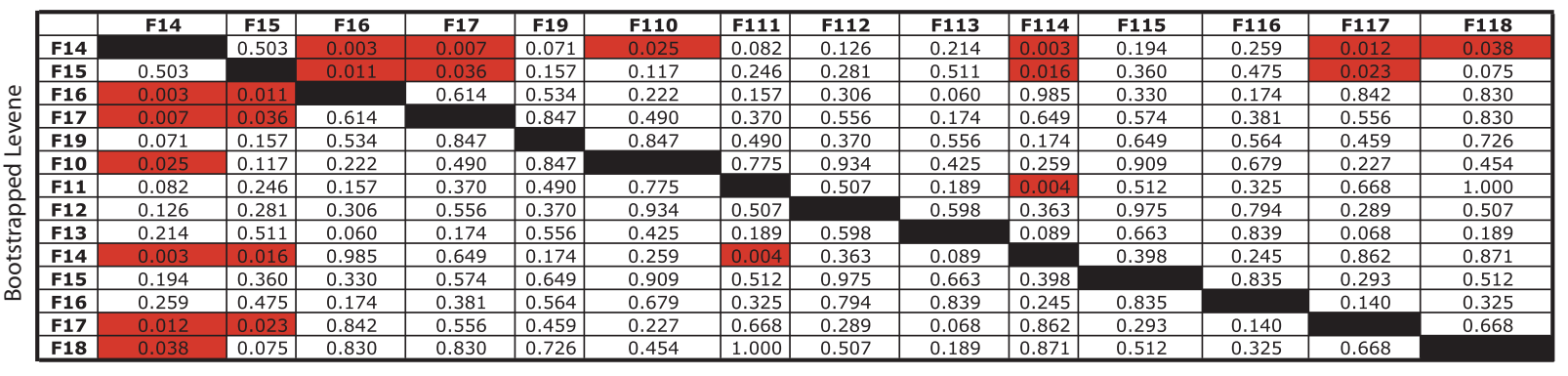




\section{Table S3}

Pairwise $\mathrm{p}$ values for Levene Test for Equal Variances (Brown-Forsythe Corrected)

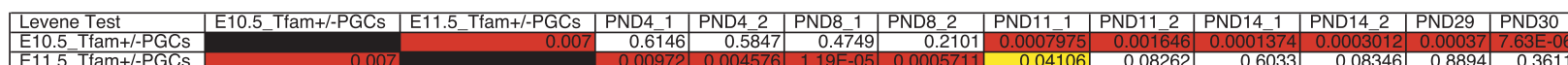

Pairwise $\mathrm{p}$ values for Bootstrpped-Levene Test for Equal Variances (Brown-Forsythe Corrected)

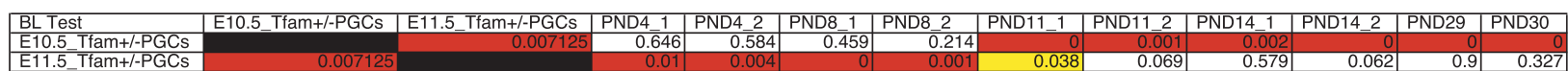


Table S4

\begin{tabular}{|c|c|c|c|}
\hline A3243G (Blood) & A3243G (Blood) & \begin{tabular}{|l|l|}
$\mathrm{T} 8993 \mathrm{G}$ (Blood) & \\
\end{tabular} & \begin{tabular}{|l|l|} 
A11778G (Blood) & \\
& .07406 \\
\end{tabular} \\
\hline$\frac{78993 G \text { (Blood) }}{\text { A11778G (Blood) }}$ & 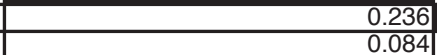 & & $\frac{0.6358}{0.635}$ \\
\hline
\end{tabular}


Chapter 3 is a manuscript to be submitted for publication, which directly test critical developmental threshold of mtDNA copy number in the embryo and oocyte of the mouse. In the construction of the various germline-specific conditional knockout mouse models, we observe a female-specific infertility phenotype in a line of mice where the mtDNA content in the ovulated oocytes is severely reducted. The data in this chapter further support the notion that the high mtDNA levels in the mature oocyte and early embryo act as a genetic mechanism to ensure the proper distribution of mtDNA in the somatic and germ cells of the next generation. 


\section{Chapter 3}

\section{The Role of Mitochondrial DNA Copy Number in Mammalian Fertility}

Timothy Wai, Xiaoyun Zhang, Asangla Ao, Daniel Dufort, and Eric A. Shoubridge 


\section{Abstract}

ATP produced by oxidative phophorylation (OXPHOS) is essential for the normal function of most eukaryotic cells. This process requires the activity of five mitochondrial multimeric enzyme complexes, which create an electrochemical gradient used to power the synthesis of ATP from ADP and inorganic phosphate. The subunits of the OXPHOS complexes are encoded by the nuclear and mitochondrial genomes (mtDNA). In mammals, the number of mitochondria and mtDNAs has been reported to vary between and within cell types, with most cells containing hundreds to thousands of copies. The most extreme variability is observed in mature mammalian gametes where oocytes contain at least 1000 times more mtDNA than sperm.

In this study, we examine the impact of mtDNA copy number on mammalian fertility by genetically manipulating copy number in the male and female germlines of the mouse. In the male germline, we report that $\mathrm{TFAM}^{+-}$males can tolerate a 3-fold decrease in mtDNA copy number in their sperm without impaired fertility or sperm function. Given that mature mouse sperm contain an average of 100 copies of $\mathrm{mtDNA}^{154}$, these studies indicate that sperm can function normally with as little as 30 copies of mtDNA. To our surprise, we observed a significant allelic imbalance with preferential transmission of the null TFAM allele when we analyzed the proportions of wild type and null TFAM allele in sperm and live offspring of these heterozygous males. In the female germline, we provide direct evidence to demonstrate a critical developmental threshold of approximately 50,000 copies of mtDNA in the mature 
oocyte. We report that both fertilization and preimplantation development can proceed normally even when mtDNA content is as low as 50,000 copies, lending strength to the hypothesis that the high mtDNA copy number present in the mature mammalian oocyte is simply a genetic mechanism that ensures that there are a sufficient number of templates present in the cells of the embryo until the time mtDNA replication restarts. 


\section{Introduction}

Studies in a variety of species have revealed that the mitochondrial content of mature female gametes is several orders of magnitude greater than in male gametes. These observations led to the notion that the strict maternal inheritance of mitochondrial DNA (mtDNA) is based on a sex-specific discrepancy in mitochondrial content. Although paternal inheritance of mtDNA can be observed in inter-specific crosses ${ }^{105,155}$ maternal germline transmission of mitochondrial DNA has been faithfully conserved throughout metazoan evolution with very few exceptions ${ }^{113,156,157}$. Selective destruction of paternal mitochondria within the fertilized egg was demonstrated to occur via ubiquitin-mediated degradation ${ }^{104,111}$. In contrast to the mitochondrial amplification that occurs during female germline development, the downregulation of key regulators of mitochondrial DNA during spermatogenesis results in a decrease in mtDNA copy number in sperm ${ }^{158-161}$. The evolutionary impetus behind the conservation of high mtDNA copy number in the female gamete and low mtDNA copy number in the male gamete, however, remains unexplained.

Despite the abundance of mitochondria in mammalian oocytes, studies of mitochondrial morphology suggest that they have little capacity for oxidative phosphorylation ${ }^{141}$. These mitochondria contain very few cristae, which are the inner membrane invaginations that harbour the five multimeric protein complexes of the oxidative phosphorylation system. Cristae increase the surface area of the inner mitochondrial membrane and are therefore abundant in mitochondria from highly aerobic and energy-demanding somatic tissues. 
Studies in frogs ${ }^{161}$, mice $^{15,23}$, rats ${ }^{162}$, shrimp ${ }^{163}$, and pig ${ }^{133}$ report that mitochondrial DNA replication does not occur during the cleavage stages of embryogenesis, which would suggest that the mitochondrial content of the oocyte is sufficient to maintain vertebrate development until implantation and for the specification of the germline in mammals ${ }^{15}$. It has therefore been proposed that the increase of mitochondria and mtDNAs during oogenesis is a genetic mechanism to ensure that a sufficient number of organelles and genomes are present in the cells of the developing embryo once mitochondrial DNA replication restarts ${ }^{2}$. The reasons for the suspension of mtDNA replication in the preimplantation embryo remain unknown. So too are the nature and origin of the signals that reinitiate mitochondrial DNA replication, which is generally believed to resume after implantation but the precise timing may vary between mammals embryonic cell lineages ${ }^{133,164}$.

In the absence of mtDNA replication, one would predict post-implantation embryonic cells to become largely depleted of mitochondrial DNA by around E7.5 to E8.5. From the initial levels of mtDNA in the cleavage stage embryo and the number of cells composing the embryo proper, embryonic cells would be predicted to contain an estimated 18 copies of mtDNA at E7.0 (4510 embryonic cells), 6 copies at E7.5 (14,290 embryonic cells), and largely devoid of mtDNA by E8.5. It is therefore not surprising that the targeted deletion of genes essential to the maintenance, replication, and expression of mitochondrial DNA results in embryonic lethality between E8.5 and E10.5, presumably due to mitochondrial insufficiency ${ }^{146,165,166}$. Knockout mouse models for 
other nuclear-encoded mitochondrial genes also appear to cause lethality around the same time in embryogenesis. ${ }^{167-173}$.

Reports that high mitochondrial content can increase the quality and competence of mature mammalian oocytes ${ }^{174}$ corroborate the notion of a critical developmental threshold, which has been proposed to be on the order of 100,000 mitochondria in humans ${ }^{98}$ and mice ${ }^{23}$. In humans, maternal age and oocyte mtDNA copy number have been reported to be negatively correlated ${ }^{175}$, and oocytes retrieved from patients with ovarian insufficiency contain on average 3 times fewer copies of $m t_{D N A}{ }^{100}$. Indeed, the link between inherited mitochondrial DNA depletion syndromes and female infertility ${ }^{176,177}$ was established when it was noticed that the inherited neurodegenerative disorders caused by a dominant mutation in the polymerase gamma gene ${ }^{178}$ also cooccurred with premature ovarian failure in females ${ }^{176,177}$, ultimately strengthening the link between defects in mtDNA and female infertility. Reduced fertility has also been documented in female mice with a proofreading-deficient version of polymerase gamma $^{179}$. It is therefore becoming clear that mtDNA copy number in the oocyte, embryo, and perhaps even the sperm ${ }^{180}$ may be playing a substantial part in the success of sexual reproduction.

Here, we investigated the role of mtDNA copy number in male and female fertility. We created germline-specific heterozygous TFAM knockout mice to reduce mtDNA content in ovulated oocytes to determine how reduced mtDNA content in these oocytes affects fertilization. By transferring the resulting low-mtDNA copy number embryos into pseudo-pregnant females we empirically determine the oocyte and 
embryonic mtDNA content necessary for development. In so doing, we also show that oocytes with mtDNA levels below the normal biological range can be successfully fertilized and proceed normally through pre-implantation development, but die during organogenesis. Conversely, we report that similar reductions in mtDNA copy number in the germline of males cause no obvious defects in fertility, sperm motility, or sperm count. 


\section{Materials and Methods}

\section{Transgenic mice and Genotyping}

All investigations were conducted in accordance with the Guide for the Care and Use of Agricultural Animals in Agricultural Research and Teaching and McGill University Animal Care Committee ethical guidelines for the care and use of laboratory animals. Heteroplasmic floxed TFAM mice were generated by crossing previously described OG2-BALB/NZB females ${ }^{141}$ to TFAM $^{\text {fl/fl }}$ males $^{146}$. F1 females were then backcrossed to TFAM $^{\mathrm{t} / \mathrm{fl}}$ males for 5 generations to generate Heteroplasmic floxed TFAM females that also carried the GOF18deltaPE transgene, henceforth termed TFAM ${ }^{\mathrm{fl} / 1 / 1}$ OG2-BALB/NZB. To generate heterozygous TFAM ${ }^{+-O G 2-B A L B / N Z B ~ a n i m a l s, ~ w e ~ c r o s s e d ~ m a l e s ~ c a r r y i n g ~}$

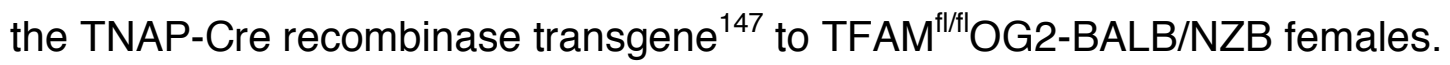
Genotyping for the presence of the GOF18deltaPE transgene ${ }^{141}$ and the TNAP-Cre recombinase transgene ${ }^{147}$ was performed as previously described. We used previously described methods for TFAM genotyping of offspring ${ }^{153}$. We determined the proportions of TFAM alleles using a modified last-cycle hot approach to incorporate gamma [ $\alpha$ $\left.{ }^{32} \mathrm{P}\right] \mathrm{dCTP}$ into the PCR amplicons generated curing the last amplification cycle. Amplicons from floxed (437bp) wild-type (404bp), and null (327bp) alleles were separated by gel electrophoresis on $12 \%$ acrylamide gels and quantified by densitometry. Radioactive intensity was normalized to amplicon length, and allelic ratios were normalized to heterozygous $\operatorname{TFAM}^{\mathrm{fl} /+}$ controls. The analysis of mitochondrial DNA heteroplasmy was performed as previously described ${ }^{141}$. 


\section{Cleavage Stage Embryo Isolation and Blastomere Biopsy}

Isolation of cleavage stage embryos and oocytes from mice was performed as previously described ${ }^{141}$. Blastomeres from 6-8 cell un-compacted and compacting embryos were isolated as previously described ${ }^{181}$. Briefly, each embryo was incubated for 5 min in $\mathrm{Ca}^{++}$and $\mathrm{Mg}^{++}$free medium (Cook Canada Inc., Stouffville, ON, Canada) to allow the blastomeres to decompact. The zona pellucida was removed using acid Tyrode's solution ( $\mathrm{pH}$ 2.5) (Sigma, Oakville, ON, Canada) and the cells washed through three drops of PBS containing $4 \mathrm{mg} / \mathrm{ml}$ bovine serum albumin (Sigma, Oakville, ON, Canada). The blastomeres were then disaggregated using a sterile pulled-glass pipette and individually pipetted into tubes containing a final volume of $10 \mathrm{ul}$ of PBS $+0.01 \%$ BSA for lysis, as downstream Q-PCR applications for the quantification of mtDNA were found to be incompatible with original lyses solutions ${ }^{181}$.

\section{Embryo Culture and Transfer into Surrogate Recipient}

Biopsied embryos were cultured until the blastocyst stage and transferred to the uteri of pseudo-pregnant recipient females as previously described ${ }^{182}$. Briefly, recipient females were mated with vasectomized CD-1 males (Charles River) and the day of plug was considered to be day 1. Heterozygous TFAM females used for embryo transfer experiments were mated with vasectomized males, and then 2 to 6 blastocysts were transferred into each uterine horn on the morning of day 4. Prior to transfer, compacted morulae were cultured in $10-\mu \mathrm{l}$ droplets of bicarbonate-buffered KSOM medium under mineral oil (Sigma) at $37^{\circ} \mathrm{C}$ in a humidified atmosphere of $5 \% \mathrm{CO} 2$ in air. After $24 \mathrm{~h}$ of 
incubation, blastocyst-stage embryos (as judged by the formation of blastocoelic cavity) were selected and transferred as described above. Uteri were recovered at E12.5 and embryos were analyzed to assess developmental progression.

\section{Sperm Collection for Motility Analyses}

12 wild type TFAM $\left(\mathrm{TFAM}^{+/+}\right.$and $\left.\mathrm{TFAM}^{\mathrm{fl} /+}\right)$ mice and 12 heterozygous $\left(\mathrm{TFAM}^{+/-}\right.$or TFAM $^{\mathrm{fl} /}$ ) mice at 4 months of age were used for the analysis of male fertility. All mice were weighed, anesthetized with isoflurane and euthanized by cervical dislocation.

\section{Sperm Motility}

Sperm motility was assessed as previously described ${ }^{183}$. Briefly, the caudal region of the right epididymidis of each mouse was placed in a microcentrifuge tube and stored at $-80 \mathrm{C}$ for subsequent sperm count analyses (see next section). The left caudal epididymidis of each animal was clamped both proximally and distally, excised, and rinsed in a 35 mm plastic Petri dish containing pre-warmed Hank's medium M199 (Invitrogen Canada, Inc., Burlington, ON) supplemented with $0.5 \%$ bovine serum albumin at $37 \mathrm{C}$. The cauda was then transferred to a fresh Petri dish, unclamped, and pierced at several points with the tip of a surgical dissection blade along the longitudinal axis of the cauda, releasing sperm into the medium. The cauda was removed and the Petri dish was placed at $37 \mathrm{C}$ for $5 \mathrm{~min}$ to allow sperm to disperse. Subsequently, an aliquot of the sperm suspension was appropriately diluted with medium and placed into an $80 \mathrm{~mm}$ 2X-CEL glass slide chamber (Hamilton-Thorne Biosciences, Beverly, MA). 
Approximately 30 scans per slide per individual mouse were analyzed using a Hamilton Thorne IVOS automated semen analyzer (Hamilton-Thorne Biosciences), using the default settings recommended by the manufacturer. Statistical and correlation analyses, and power tests of motility data, were done using Version 7.1 of the Statistica Data Miner for Windows (Statsoft, Inc., Tulsa, OK). The parameters of sperm motility are abbreviated as VAP (average path velocity), VSL (progressive velocity; i.e. average velocity measured in a straight line from beginning to end of track), VCL (track speed; i.e. average velocity measured over the actual point to point track followed by the cell), ALH (amplitude of lateral head displacement; i.e. average displacement path corresponding to average of the sperm track width), BCF (beat cross frequency; i.e. the frequency with which the sperm head crosses over the sperm average path in either direction), STR (average value of the ratio of VSL/VAP and measures the departure of the cell path from a straight line), and LIN (average value of the ratio of VSL/VCL and measures the departure of the cell track from a straight line).

\section{Sperm Counts}

Sperm counts were determined as previously described ${ }^{183}$. Briefly, the frozen right caudal epididymidis of each animal was thawed and homogenized in a $50 \mathrm{ml}$ conical tube containing $10-20 \mathrm{ml}$ of distilled water. A $100 \mathrm{ml}$ aliquot of the resulting homogenate was diluted with $100 \mathrm{ml}$ of distilled water in a $1.5 \mathrm{ml}$ microcentrifuge tube coated with "IDENT fluorescent dye" (Hamilton-Thorne Biosciences) and incubated at room temperature for $2 \mathrm{~min}$. The suspension was mixed and a $5 \mathrm{ml}$ aliquot was placed in a 20 
mm sperm analysis chamber (2X-CEL; Hamilton-Thorne Biosciences) and quantified with the IVOS semen analyzer.

\section{Mitochondria DNA Quantification}

Absolute mtDNA copy number measurements made in cleavage stage embryos and oocytes were performed as previously described ${ }^{141}$. Relative quantification of mtDNA levels measured in sperm and male reproductive tissues was determined by the ratio of the mitochondrial ND2 (mt-ND2) gene (DQ874614) to the single-copy nuclear-encoded B2M (Beta-2-Microglobulin) gene (NM_009735). Q-PCR reactions using B2M forward (5'TGTCAGATATGTCCTTCAGCAAGG3') and reverse (5'TGCTTAACTCTGCAGGCGTATG3') primers were performed using DNA Faststart Master Plus SYBR Green (Roche) on the Rotorgene 3000 (Corbett) using the following protocol: 35 cycles, denaturation at $95 \mathrm{C}$ for $10 \mathrm{~s}$, annealing at $62 \mathrm{C}$ for $15 \mathrm{~s}$, and extension at $72 \mathrm{C}$ for $20 \mathrm{~s}$. Melt-curve analyses reported on the specificity of the PCR products that were amplified. Post-run analyses of the amplification efficiency were performed and corrected using Rotorgene software and LINREGPCR programs ${ }^{134}$. Serial dilutions inputs for both the ND2 and B2M Q-PCR reactions were used to ensure identical amplification efficiencies, after which ratios for each sample were generated using arbitrary concentration units. Replicate serial dilutions within the linear range for both nuclear and mitochondrial genes were used to calculate relative mtDNA copy number. 


\section{Statistical Testing}

Chi-square tests were used to asses the difference in survival rates between low copy number TFAM ${ }^{+/-}$embryos and high copy number TFAM ${ }^{+/}$embryos, between the number of wild type and deleted TFAM alleles segregating in testcrosses and present in sperm samples from TFAM ${ }^{+/}$males. Groups producing $p$ values $<0.05$ were considered significantly different. 


\section{Results}

\section{Defining the Developmental Threshold of Mitochondrial DNA in the Early Embryo}

Although a critical threshold of oocyte mtDNA content ( 100,000 copies), is thought to be required for fertilization and embryonic development in mammals ${ }^{23,100,174,184}$, to our knowledge there is no direct experimental evidence to support this contention. In order to determine the critical developmental threshold, we first investigated the biological variability in mtDNA content in the mature oocyte of the mouse. To avoid potential contamination by sperm and cumulus cells, we measured the absolute mtDNA copy number in 2,4 , and 8 cell embryos generated by natural timed matings between proven breeders. In the absence of pre-implantation mtDNA replication, the levels of mtDNA in the mature oocyte accurately reflect those of the cleavage stage embryo ${ }^{5}$. In addition, damaged or poor quality oocytes unable to perform the initial cleavage divisions, and destined for destruction, were excluded from these analyses. We did not super-ovulate prior to copulation since it has been reported that gonadotropins (e.g. hCG) can inflate ATP levels and mtDNA copy number rodents $^{185}$. We analyzed 219 cleavage stage embryos and found an average of 161,000 $\pm 73,000$ copies of mitochondrial DNA (Figure 1) (median: 154,000 copies, range:

11,000 to 428,000 copies). Further analysis showed an asymmetry in the distribution of copy number in these embryos (skewness $=0.534$, kurtosis $=0.317$ ) with only 3 out of 219 embryos $(1.4 \%)$ from this non-normal distribution $(p<0.0001)$ containing fewer than 40,000 copies and $95 \%$ of embryos containing between 50,000 and 330,000 copies (Figure 1), making it likely that the single 11,000 copy number embryo be an outlier. 


\section{Oocyte fertilization and cleavage are not affected by low mtDNA content}

Previous reports linking oocyte competence and mitochondrial content suggested that low mtDNA content could prevent fertilization ${ }^{186}$. We sought to determine whether low mtDNA levels in the oocyte could directly affect its ability to be fertilized by sperm. As a source of oocytes containing decreased amounts of mtDNA, we elaborated on a previously used heteroplasmic mouse model in which we deleted a single copy of the TFAM gene using Cre-recombinase mediated germline excision of a floxed allele by crossing TNAP-Cre ${ }^{+/+}$males to a TFAM ${ }^{\text {fl/fl }}$ heteroplasmic females. Our TFAM ${ }^{+/-}$females, born to these F1 TFAM ${ }^{\mathrm{fl} /+}$;TNAP-Cre ${ }^{+}$mothers, generated cleavage stage embryos (and therefore mature oocytes) that contained an average of 69,000 copies of mtDNA (Figure 2a) (approximately $40 \%$ of control), which is similar to the previously reported decrease in mtDNA content observed somatic tissues of heterozygous TFAM adults and embryos. Reproduction in both males and females was normal, with normal litter sizes and numbers obtained in reciprocal testcrosses as well as intercrosses. However, in females whose germlines were made heterozygous for TFAM by Zp3-Cre recombinasemediate excision during folliculogenesis $\left(\operatorname{TFAM}^{\mathrm{fl} /+} ; \mathrm{Zp} 3-\mathrm{Cre}^{+}\right)$, we observed dramatically reduced female fertility. Interestingly, these females generated 2-cell zygotes that contained an average of 18,000 copies of mtDNA per embryo (approximately $11 \%$ of control) (Figure 2B). Zp3-Cre recombinase is active exclusively in the female germline, during oocyte growth. As predicted, we did not observe male infertility in TFAM ${ }^{\mathrm{fl} /+}$; Zp3$\mathrm{Cre}^{+}$male littermates. Since the number of cleavage stage embryos isolated from these same infertile TFAM ${ }^{\mathrm{fl}+}$; Zp3-Cre ${ }^{+}$females (mated to fertile males) was not significantly 
different than control (DNS), it was not possible to attribute the observed infertility to either aberrant ovulation or oocyte fertilization. We examined mtDNA copy number in another knockout mouse model for MPV17, which is a gene that, when mutated in humans, causes an autosomal recessive infantile liver-specific mitochondrial DNA depletion syndrome ${ }^{187}$. We did not observe a decrease in mtDNA copy number in embryos from MPV17 ${ }^{-/}$females ( $n=9$ ), which generated offspring and litter sizes comparable to controls. To our surprise, $\mathrm{COX}_{10^{+/-}}$(but not COX10 ${ }^{\mathrm{fl} /+} ; \mathrm{Zp} 3-\mathrm{Cre}{ }^{+}$or COX10 $\left.{ }^{\mathrm{fl} /+} ; \mathrm{Zp} 3-\mathrm{Cre} \mathrm{e}^{-}\right)$females exhibited a 2.5 fold increase in mean mtDNA copy number in cleavage stage embryos (Figure $2 \mathrm{C}$ ), but this did not affect either litter size or the number of ovulated oocytes (DNS). Like most other genes coding for factors required for the proper assembly of the respiratory chain complexes, homozygous deletion of COX10 in the mouse is incompatible with life ${ }^{172}$.

We occasionally observed developmental asynchrony in a minor fraction of embryos isolated between 0.5 to $3.5 \mathrm{dpc}$, both from wild type and all knockout females, but the rate of progression through cleavage did not correlate with mtDNA copy number. Taken together, these data argue strongly that low mtDNA copy number does not significantly impede either fertilization or pre-implantation development in the mouse.

\section{Low mtDNA copy number impairs post-implantation development}

In order to determine whether decreases in post-implantation viability and live birth number were directly related to the amount of mitochondrial DNA inherited from the oocyte, we transferred TFAM ${ }^{+/}$blastocysts into pseudo-pregnant wild-type females, and 
assessed the developmental outcome of these low mtDNA copy number embryos at either 10.5 or $12.5 \mathrm{dpc}$. Fertilized oocytes and cleavage stage embryos from TFAM ${ }^{+/-}$ females contain about 69,000 copies of mtDNA (Figure 2A). However, as the distribution of mtDNA copy number in $\mathrm{TFAM}^{+/-}$embryos overlaps with the normal distribution observed in wild-type embryos (Figure 2), it was not possible to simply transfer TFAM ${ }^{+-}$embryos; we needed to determine individual mtDNA content of these low-copy number blastocysts prior to transfer. To do so, we quantified mtDNA copy number by Q-PCR from single blastomeres that were biopsied from 8-cell $\mathrm{TFAM}^{+/}$ embryos. This allowed us to estimate the total embryonic content of mtDNA in these embryos, which we cultured overnight before performing the transfer (Table 1). Serving as controls, higher copy number $\mathrm{TFAM}^{+/-}$embryos were transferred to the right uterine horn while low copy number embryos were transferred to the left, allowing us to distinguish an implantation failure unrelated to embryonic content of mtDNA. We transferred 19 high copy number TFAM ${ }^{+/-}$embryos and 15 low copy number $\mathrm{TFAM}^{+/-}$ embryos into 4 surrogate females (Figure 3). Only 2 out of 19 (11\%) low-copy number embryos developed normally (including 1 our of 2 low-copy number embryos in group 7) compared to 9 out of 15 high-copy number embryos (60\%). In our hands, we obtain an $80 \%$ survival rate of $2.5 \mathrm{dpc}$ embryo transfers performed without biopsy of blastomeres and overnight culture, which is not significantly different from the survival rate measured in the TFAM+/- embryos serving as controls (Chi-squared test, $p=0.528$ ). However, we observed a dramatic reduction in post-implantation embryonic viability in the context of the low-copy number TFAM+/- embryos (Chi-squared test $\mathrm{P}<0.001$ ) (Figure 4). From 
these studies we conclude that an oocyte mtDNA content below $\sim 40-50,000$ copies (i.e. the low end of the biological distribution found in wild-type oocytes) is unlikely to progress normally through post-implantation development. It is worthwhile noting that while we did observe a single sub-threshold embryo (group 7; 26,000 copies) develop normally at E10.5, this discrepancy can be accounted for a single additional round of mtDNA replication and may represent a mechanism that "senses" boardline embryos (this may be a little far-fetched...can we discuss?).

\section{Reduction in mtDNA content in sperm does not affect function}

We failed to observe overt signs of infertility in $\mathrm{TFAM}^{+/-}$males, such as the inability to copulate, absence of vaginal plugs, or decreased litter sizes. However, the suggested link between mtDNA and male fertility reported in asthenozoospermic and oligozoospermic humans, ${ }^{188,189}$, and in mice carrying large-scale deletions of mtDNA ${ }^{190}$, led us to test whether a more subtle functional impairment could manifest in the sperm of $\mathrm{TFAM}^{+/-}$males, which contain mtDNA levels that contain significantly less mtDNA (Student's t-test $p<0.001$ ). We assessed several standard measures of motility in sperm isolated from the caudal epididymis of TFAM heterozygous and wild type littermates and observed no significant differences (Figure 5A). We also failed to detect differences in sperm count in these animals, despite a significant 3-fold relative reduction in mtDNA content. It is perhaps worthwhile to note that we observed greater variability in both sperm motility and sperm count among wild-type males born to 
mothers of different nuclear genetic backgrounds than between wild type and $\mathrm{TFAM}^{+-}$ littermates (DNS).

To exclude the possibility that the normal sperm function and count were the result of an allelic imbalance, namely the over-representation of wild-type sperm, we performed quantitative genotyping of TFAM alleles on DNA extracted the caudal epididymis sperm and from the male reproductive tract (epididymis and testes). To our surprise, we observed a significant allelic imbalance in favor of the deleted TFAM allele in the sperm (Figure 5B) that was reflected in offspring generated from recessive testcrosses between heterozygous TFAM males and wild type females. Live offspring generated from 7 separate recessive testcrosses demonstrated that of a total of 114 inherited TFAM alleles, 80 of those alleles were TFAM - (compared to an expected 62 alleles). In other words, approximately $65 \%$ of the time the offspring inherited the deleted allele from a heterozygous father rather than the Mendelian prediction of $50 \%$. When the reciprocal testcross of wild type males to heterozygous females was performed, the deleted allele was inherited at a frequency of 94 alleles out of a possible 193 alleles (53\%), indicating that allelic imbalance is a male-specific trait. 


\section{Discussion:}

In this study we test the existence of a developmentally important threshold of mtDNA copy number in the mature, ovulated oocyte of the mouse. While live offspring cannot be generated from the fertilization of oocytes with sub-threshold levels of mtDNA, we demonstrate that fertilization and pre-implantation development can proceed unimpeded. Consequently, the embryonic defect that was first recognized as decreased female fecundity in a line of heterozygous TFAM animals (which exhibit a reduction in mtDNA copy number) occurs during post-implantation development. We provide evidence from embryo transfer experiments that demonstrate that a reduction in mtDNA copy number in the female germline below 50,000 copies of mtDNA per mature oocyte is largely incompatible with normal embryonic development. On the other hand, a three-fold reduction in mtDNA copy number in male gametes does not affect function (sperm count, sperm motility, male fertility) even though spermatozoa normally contain 100 mtDNAs and rely on OXPHOS to power movement.

\section{Low mtDNA content does not prevent fertilization}

We demonstrate that reduced mtDNA copy content in sperm from TFAM heterozygous males does not impede fertilization per se, although it is clear that the generation of ATP by OXPHOS is essential for sperm motility ${ }^{189}$. TFAM heterozygous males have sperm counts and motility parameters indistinguishable from their wild-type littermates. However, sperm from these animals contain 3 times less mtDNA. Based on studies reporting an average of $100 \mathrm{mtDNAs} / \mathrm{sperm}$, this would mean that mature 
murine sperm can function normally with as few as 33 copies of mtDNA. While further studies are required to define the minimal copy number requirements for both spermatogenesis and fertilization of the egg, our results indicate that mature murine sperm contain more than the necessary number of mitochondrial genomes required to power their movement. Supporting this idea is a study demonstrating that mouse mtDNA molecules with large-scale deletions cause male-related infertility only when the mutant load is high ${ }^{190}$. In these mito-mice, impaired spermatogenesis and sperm number and motility begins to manifest once the mutant load exceeds $73 \%$; presumably leaving an estimated 27 functional copies of mtDNA in the mature sperm, which is similar to our study. However, it is unclear to what degree changes in behaviour contribute to this decreased reproductive capacity, given the incapacitating nature of the mitochondrial myopathy whose severity increases as a function of mutational load. It is also difficult to determine the residual function, if any, retained by partially deleted mtDNA molecules in the context of sperm function.

It is tempting to propose a critical mtDNA threshold for sperm function and perhaps male fertility based on these data, but we caution against this impulse since the mtDNA copy number decrease in sperm from TFAM heterozygous animals is also extended to the testes and caudal epididymis tissues. The development of the soma and germline in both sexes are deeply intertwined at virtually all stages of gametogenesis and the proper maturation of sperm is critically dependent upon proper development and function of both the sertoli and leydig cell populations present in the testes. In addition, fertilization is also contingent upon proper development of the male reproductive tract. 
Defining the crucial threshold of mtDNA content in the sperm will not be so straightforward, given the intricate interplay between germline and soma. We therefore propose that the mtDNA content of both mature sperm and reproductive tract tissues is in excess of that required for normal gametogenesis. This hypothesis is consistent with our reports of allelic imbalance in sperm generated from TFAM heterozygous males (either TFAM ${ }^{\mathrm{fl} /-}$ or $\mathrm{TFAM}^{+-}$) and the offspring generated from recessive testcrosses. Male transmission of a deleted TFAM allele occurs more often than would be predicted by chance alone $(p<0.0001)$, suggesting that the diminished content of mtDNA accompanying that deleted allele exercises some kind of functional advantage at some point during spermatogenesis. It is not immediately evident how a relatively lower number of genomes would be advantageous to the developing spermatozoa, although it seems as though evolutionary design has invoked a downregulation of both POLG and TFAM to yield a decrease in mtDNA copy number during spermatogenesis ${ }^{159,160}$. Oligospermic and asthenozoospermic men have sperm that contain significantly elevated levels of mtDNA ${ }^{100,191}$, prompting the hypothesis that reduced copy number in sperm decreases the likelihood of ROS-mediated damage to mtDNA, effectively mitigating potentially deleterious effects on sperm function. However, little compelling evidence exists to lend credence to the ROS hypothesis and if an upper limit of mtDNA content in mature spermatozoa truly exists, its successful definition will likely rest in the genetic manipulation of animal models with more uniform nuclear genetic backgrounds. Female germline manipulation of mtDNA copy number has allowed us to vary mtDNA copy number to a much greater degree. Despite severe reductions in mtDNA copy 


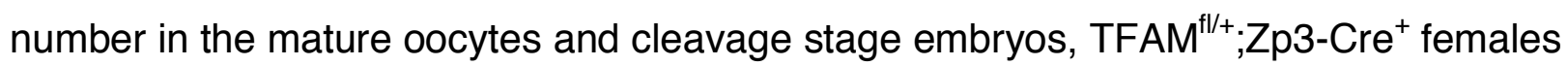
still produced normal numbers of fertilized oocytes, cleavage stage embryos, and live offspring, which indicate that neither fertilization nor pre-implantation development is affected. We failed to connect developmental asynchrony in the pre-implantation embryo to mtDNA levels in embryos generated from either the germline-specific $\mathrm{TFAM}^{+-}$ or the TFAM ${ }^{\mathrm{tl} /-}$, which generated litter sizes and numbers similar to controls. These data argue strongly that low mtDNA copy number in the ovulated oocyte does not negatively impact fertilization or pre-implantation development, suggesting that the high mtDNA levels observed in the mature oocytes of most mammals does not play a functional role during this period of development. 


\section{The Developmental Threshold of mtDNA and Post-implantation Viability}

Several reports of high mitochondrial number estimated in oocytes from a variety of mammals has led to the notion that there exists a critical threshold of mitochondrial DNA copy number required for the successful fertilization and development of the embryo. However, this number has yet to be defined and directly tested. It seems reasonable to speculate that the biological range in mtDNA content of the mature oocyte represents the viable amount that has been empirically determined by mammalian evolution. In humans, estimates of mtDNA copy number in presumably healthy metaphase II human oocytes range from 50,000 to $1,500,00^{96-100}$. While this range is much greater than what we observed in mice (Figure 1; range 11,000 to 428,000 copies per embryo), we found that the low end of the distribution coincided with the human data. In fact, we found very few presumably viable embryos to contain less than 50,000 copies of mtDNA and only 4 out of $219(1.8 \%)$ to contain 40,000 or fewer copies of mtDNA (these embryos each contained 11000, 24000, 30,000, and 40,000 copies). To test whether the observed low end of this distribution represents a functionally relevant critical threshold, we determined mtDNA copy number in embryos derived from transgenic females carrying a deleted copy of TFAM (a gene required for the replication and maintenance of mtDNA) and transferred these blastocysts to recipient surrogate females. Cleavage stage embryos isolated from these $\mathrm{TFAM}^{+/-}$female contained mtDNA levels (range 3,000 to $120,000)$, which was lower than the mean of our controls. Estimates of mtDNA content of these $\mathrm{TFAM}^{+/-}$embryos were calculated from the mtDNA levels 
measured in individual blastomeres biopsied at the 8-cell stage. Despite the invasive nature of this procedure, greater than $90 \%$ of biopsied embryos were successfully cultured to the blastocyst stage and transferred to the uterine horns of pseudo-pregnant recipient females. Of these cultured embryos with mtDNA levels above the hypothetical critical threshold, approximately $60 \%$ implanted and developed normally. We attribute this difference, while not statistically significant (Chi-squared test $p=0.528$ ), to the invasiveness of the manipulations that require disruption of the zona pellucida, the biopsy of individual blastomeres, and lengthy in vitro culture. Therefore, from these data we conclude that the minimal mtDNA copy number in the oocyte that is required for post-implantation development, and hence necessary for embryonic development as a whole is approximately 50,000 copies. Since mtDNA replication does not restart until the egg cylinder stage (about E6.0 in the mouse) ${ }^{15}$, embryos at the critical copy number threshold would be predicted to contain an average of 1 to 2 mtDNA molecules (and likely 1 organelle) per cell ${ }^{141}$, based on the naive assumption that mtDNAs are continuously and uniformly distributed to the cells of the developing postimplantation embryo. Nevertheless, from these data we conclude that the critical threshold for mtDNA copy number in the mature mouse oocyte has evolved on the basis of a functional consequence of mitochondrial insufficiency in the postimplantation embryo, lending strength to the claim that high mtDNA content in the mature female gametes is a genetic mechanism ensuring the faithful transmission of these organelles to all the cells of the developing embryo. 
However, the upper end of the biological range in wild type embryos does not appear to define critical upper limit of mtDNA copy number. COX10 $10^{+-}$ heteroplasmic females generate normal embryos with a mean copy number that is equal to the highest copy number found in wild type embryos (Figure 2). Our copy number measurements in embryos from $\mathrm{COX} 10^{+/-}$females (mean 423,000

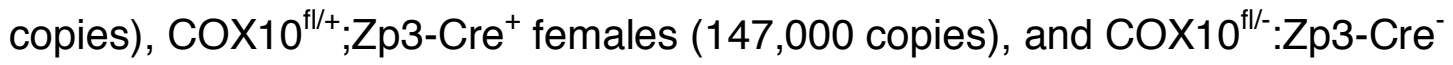
(151,000 copies) indicate that an early, but not late, heterozygous germline deletion of COX10 leads to an upregulation of mtDNA copy number, which is a typical compensatory response to OXPHOS deficiency adopted by many cell types. Mitochondrial hyper-proliferation has been documented in affected tissues from patients with inborn errors of OXPHOS (e.g. COX or Complex IV deficiency) ${ }^{192}$. However, when mutations are discovered in nuclear genes, such as those that encode COX assembly factors (e.g. COX10, COX15) their transmission follows an exclusively autosomal recessive pattern, presumably due to haplosufficiency ${ }^{193-196}$. In this study, we report that the soma and germline of $\mathrm{COX} 10^{+/-}$animals are clearly affected and therefore call into question the happlosufficiency of COX10. Given the absence of truly null alleles in enzymatic assembly factors for the respiratory chain, we propose that the purported haplosufficiency of human COX10, as evidenced by asymptomatic carries, actually reflects the residual function of the COX10 missense alleles in the carriers. Moreover, the live births of compound COX10 heterozygotes, which have about $30 \%$ of residual COX activity ${ }^{193}$, speaks to the residual function of these alleles that clearly yield sufficient ATP for in utero development. COX10-/- 
null human embryos, like their murine counterparts, likely succumb to spontaneous abortion after implantation, due to mitochondrial insufficiency. In this study, we genetically manipulate germline mtDNA copy number (range: $11,000$ to $1,400,000)$ and show that both fertilization and pre-implantation development can proceed unhindered, even in low-copy number embryos fated to abort following implantation. Coupled with the fact that the deletion of nuclear genes encoding factors essential to the biogenesis of mitochondria often cause death after implantation, we conclude that the checkpoints for implantation, cleavage, fertilization, and, to a certain degree, ovulation are not strictly regulated by mitochondrial metabolism or copy number.

\section{Clinical and Evolutionary Relevance}

The machinery involved in mitochondrial DNA maintenance, replication, and gene expression $^{8}$ has taken on new clinical significance ever since mutations the in the nuclear-encoded gamma DNA polymerase dedicated to the replication of mtDNA were proven to cause progressive and adult-onset neuropathies ${ }^{178}$. Since then, both loss-of-function and dominant negative mutations in POLG have been demonstrated to manifest in a variety of different metabolic disorders, which include a recurring theme of mitochondrial DNA depletion ${ }^{197}$. It is therefore not surprising that the original Y955C dominant POLG mutations responsible for disrupting the faithful replication of mtDNA in somatic tissues also cause familial forms of female reproductive disorders ${ }^{176,177}$. Anecdotal evidence of POLG patients seeking treatment by way of in-vitro fertilization (IVF) or other assisted 
reproductive technologies (ART) highlights the importance of defining the critical threshold of mtDNA in the general human population (personal communication, M. Zeviani). As in the mouse, it is likely that the normal biological range of mtDNA copy number in the oocytes of healthy fertile females defines the critical developmental threshold. While it is unlikely that the etiology of female-related infertility is entirely attributable to insufficient levels of mtDNA in the ovulated oocyte, we propose that mtDNA copy number should be examined when selecting ex-vivo cultured embryos for uterine transfer, particularly when blastomere biopsies are performed for the purposes of traditional pre-implantation genetic diagnoses. Regardless of the mean copy number, the lessons from our conditional knockout mouse models indicate a broad biological range (Figure 2). It would be prudent to transfer and implant embryos with the highest likelihood of successful development, which may be contingent upon mtDNA content in some cases. Future human studies will be needed to evaluate the developmentally and clinically-relevant threshold of mtDNA.

Irrespective of the minimal number of genomes required for human postimplantation development, the question still remains: why did evolution insist upon a flurry of mitochodnrial biogenesis within the growing postnatal follicle to yield a mature oocyte with tens or hundreds of thousands of copies of mtDNA, especially when the absence of continued mtDNA replication during preimplantation development ultimately reduces copy number to original levels at the time of embryogenic germline specification? It is clear that elevated levels of mtDNA are neither paramount to fertilization nor cleavage. In addition, we have 
recently demonstrated that the physical bottleneck created at the outset of gametogenesis is not involved in the rapid germline segregation of mtDNA sequence variants in the mouse ${ }^{141}$.

It has been proposed that the logic behind the inflation and deflation of mtDNA levels in the germline lies in the selective elimination of severely deleterious mtDNA mutations ${ }^{131,141}$. In order for the so-called filter for purifying selection to act during embryonic (female) germline development, it must be set in the context of low organellar content of mtDNA copy number in the primordial germ cells. Beginning embryogenesis with high levels of mtDNA and then halting replication during pre-implantation development would allow the incipient primordial germ cell population to effectively filter out severely deleterious mtDNA mutations ${ }^{130}$, which are predicted to occur 10-20 times more frequently than in the nucleus ${ }^{198}$. In the absence of an efficient DNA repair system, protective histones, and recombination, ROS-mediated damage of mtDNA, the mitochondrial genome would rapidly accumulate mutations and become entirely non-functional. Indeed, mice lacking the proof-reading function of the mitochondrial DNA polymerase accumulate somatic mtDNA mutational loads orders of magnitude greater than in wild type mice, and display symptoms of premature ageing ${ }^{179}$.

Hence, evolution seems to have devised a system balancing elevated levels of mtDNA in the mature oocyte that can sustain an exponential decrease in mitochondrial content that halted mtDNA replication causes, allowing mtDNA levels to dip to the levels required for purifying selection to act at the level of the 
organelle in the developing germline, yet not so low that it compromises postimplantation development (Figure 6). Nevertheless, even the most severely deleterious mutations can sneak through this purifying filter, which sometimes required several generations of selection to be completely eliminated. In fact, the majority of inherited forms of mitochondrial DNA diseases in humans, which occur at a frequency of 1 in 5000 , are caused by mutations that are not effectively eliminated by this system. The mechanism responsible for the elimination of mtDNA mutations has yet to be identified, but the study of spontaneous mutations in the germlines of mice carrying an error-prone mtDNA polymerase have lead us to advance a hypothesis that germline selection against severe mutations acts at the level of the organelle. We have proposed that a functional discrepancy between mitochondria carrying wild type versus mutated genomes exerts a slow but consistent advantage during the 15 mitotic divisions of the developing germline. We submit that the selective elimination of severely deleterious mutations would be most efficient when the mitochondrial content of mtDNA is lowest since clear differences in organellar fitness would manifest more quickly, particularly during the constant replication of mtDNA needed to keep step with the mitotic divisions of the germline during oogenesis. If selection were based on an indicator of mitochondrial function (e.g. membrane potential), which could be compromised by severe mtDNA mutations, the embryonic increase in the number of cells and mitochondrial genomes would provide a continuous opportunity to ensure that the developing germline were populated by the fittest mitochondria (i.e. highest membrane potential). Experimentally validation of whether the filter 
for purifying selection of severe mtDNA mutations is in fact contingent upon a physical mtDNA bottleneck in embryogenesis may provide clues as to the mechanistic basis of selection. 


\section{Figure Legends}

Figure 1: Distribution of mitochondrial DNA copy number in wild type embryos

Mitochondrial DNA copy number in wild type heteroplasmic cleavage stage embryos contain an average of $161,000 \pm 73,000$ copies of mtDNA per embryo $(n=219)$ and range between 11,000 to 428,000 copies. $95 \%$ of all embryos (208/219) contain between 50,000 and 330,000 copies.

Figure 2: Distribution of mitochondrial DNA copy number in germline knockout embryos

Comparison of mitochondrial DNA copy number in wild type heteroplasmic cleavage stage embryos to TFAM ${ }^{+-}$heteroplasmic females (A), $\operatorname{TFAM}^{\mathrm{fl} /+} ; \mathrm{Zp} 3-$ $\mathrm{Cre}^{+}$heteroplasmic females (B), and $\mathrm{COX} 10^{+/-}$females (C). Embryos from $\mathrm{TFAM}^{+/-}$heteroplasmic females (A, black) contained an average of 69,000 copies of mtDNA ( $n=74)$ while those from TFAM ${ }^{\mathrm{fl} /+} ; \mathrm{Zp3}-\mathrm{Cre}^{+}$heteroplasmic females (B, black) contained an average of 18,000 copies $(n=96)$. Embryos from heteroplasmic $\mathrm{COX} 10^{+/-}$females (C, black) contained an average of 424,000 copies of mtDNA $(n=54)$.

Figure 3: Defining the Critical Developmental Threshold of mtDNA Copy Number Mitochondrial DNA quantification of biopsied blastomeres from cleavage stage embryos (8-cell) was performed in order to estimate mtDNA content in cleavage 
stage embryos before transferring to surrogate recipients. Embryos were grouped into Low Copy or High Copy groups and transferred to separate uterine horns of the a surrogate. In some cases, it was possible to assign copy number in individual embryos, by examining the heteroplasmy measurements in the developed post-implantation embryo to the biopsied blastomere. Normal, stageappropriate morphologically development were scored as positive while aborted and developmentally delayed embryos were scored as negative. All images were acquired under the same magnification (25X).

\section{Figure 4: Embryo transfer and developmental outcome}

Bar graphs represent the mean frequency of implantation and survival, scored by normal morphological and developmental progression following embryo transfer of non-biopsied controls, Low Copy group embryos ( $n=19$, mtDNA copy number range: 3,558 to 55,000$)$, and High Copy group embryos ( $n=15$, mtDNA copy number range: 56,000 to 160,000 ). Low Copy and High Copy groups were determined to be statistically different $(p>0.001)$ but the developmental outcome between and the non-biopsied controls embryos and the High Copy group were no different $(p=0.52)$.

Figure 5: Measurement of Sperm Function and Allelic Bias in TFAM heterozygous males

Standard measures of sperm motility (VAP, VSL, VCL, ALH, BCF, STR, LIN, \% motile) and sperm count were assessed in caudal epididymal samples from 
TFAM heterozygous males (A). Bar graphs represent the results as a percentage of wild-type littermate controls of VAP. No statistically significant differences (student's t-test) were observed between groups for any of the measurements. A paternal allelic bias of the deleted TFAM allele from $\mathrm{TFAM}^{+-}$males was observed in these sperm samples as well as in the offspring of recessive testcrosses (B). Floxed TFAM, wild type TFAM, and maternally transmitted deleted TFAM alleles segregated in Mendelian fashion.

Figure 6: Critical Threshold of mtDNA Copy Number during

\section{Embryogenesis}

The model for the evolution of mtDNA copy number in the female germline indicates a critical threshold for mtDNA set at approximately 50,000 copies of mtDNA in the mature oocyte. Sub-threshold embryos can progress normally through cleavage but spontaneously abort after implantation. At E6.0, which is the stage at which mtDNA replication resumes, the cells of the embryo proper would be expected to contain an average of 215 copies, assuming equal distribution of mtDNA in the cells of the developing embryos. TFAM ${ }^{\mathrm{fl} /} ; \mathrm{Zp} 3-\mathrm{Cre}^{+}$ oocytes ( 18,000 copies of mtDNA) (red) are successfully fertilized and progress through cleavage but fail to develop normally following implantation, due to mitochondrial insufficiency. 
Figure 1

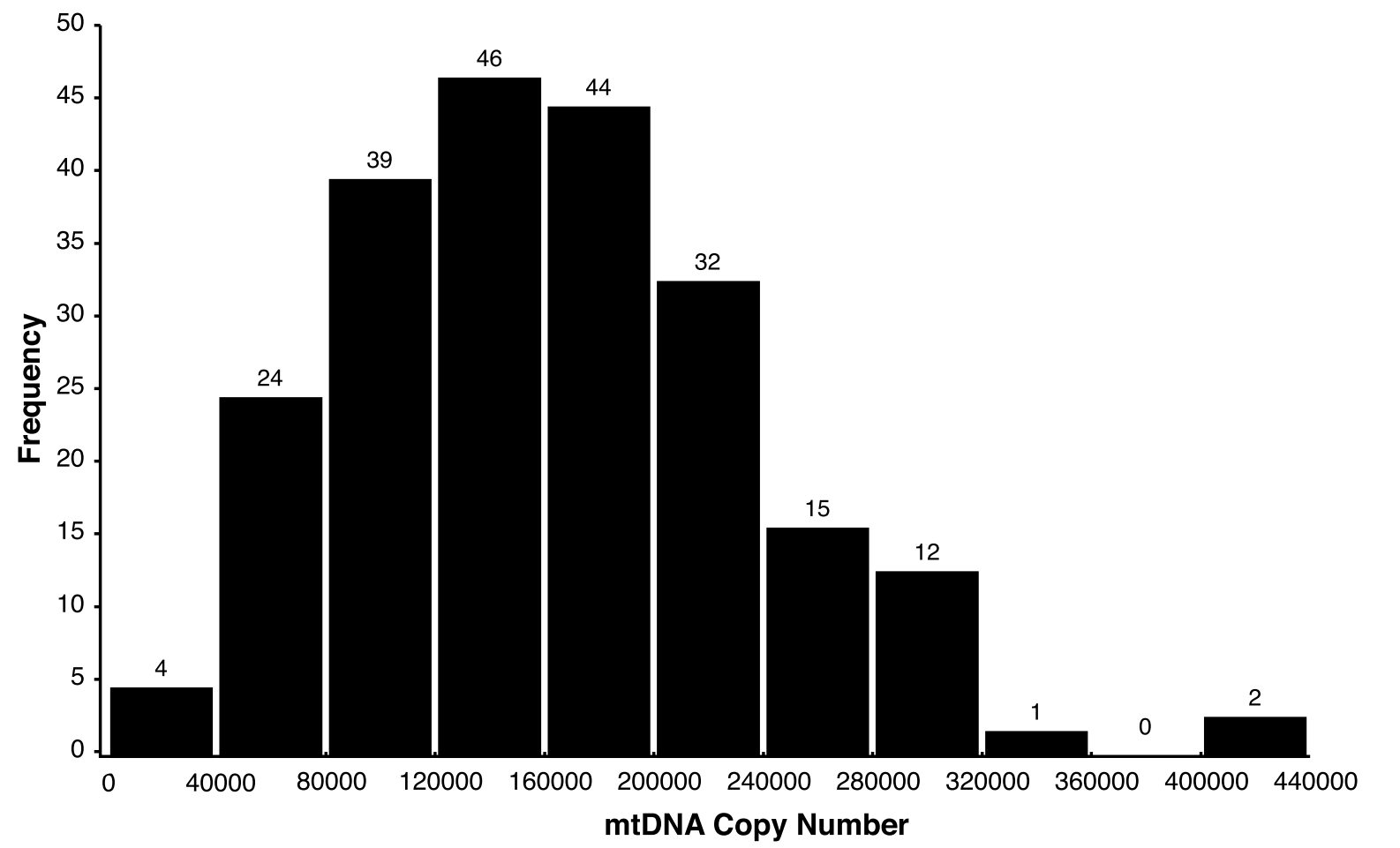




Figure 3

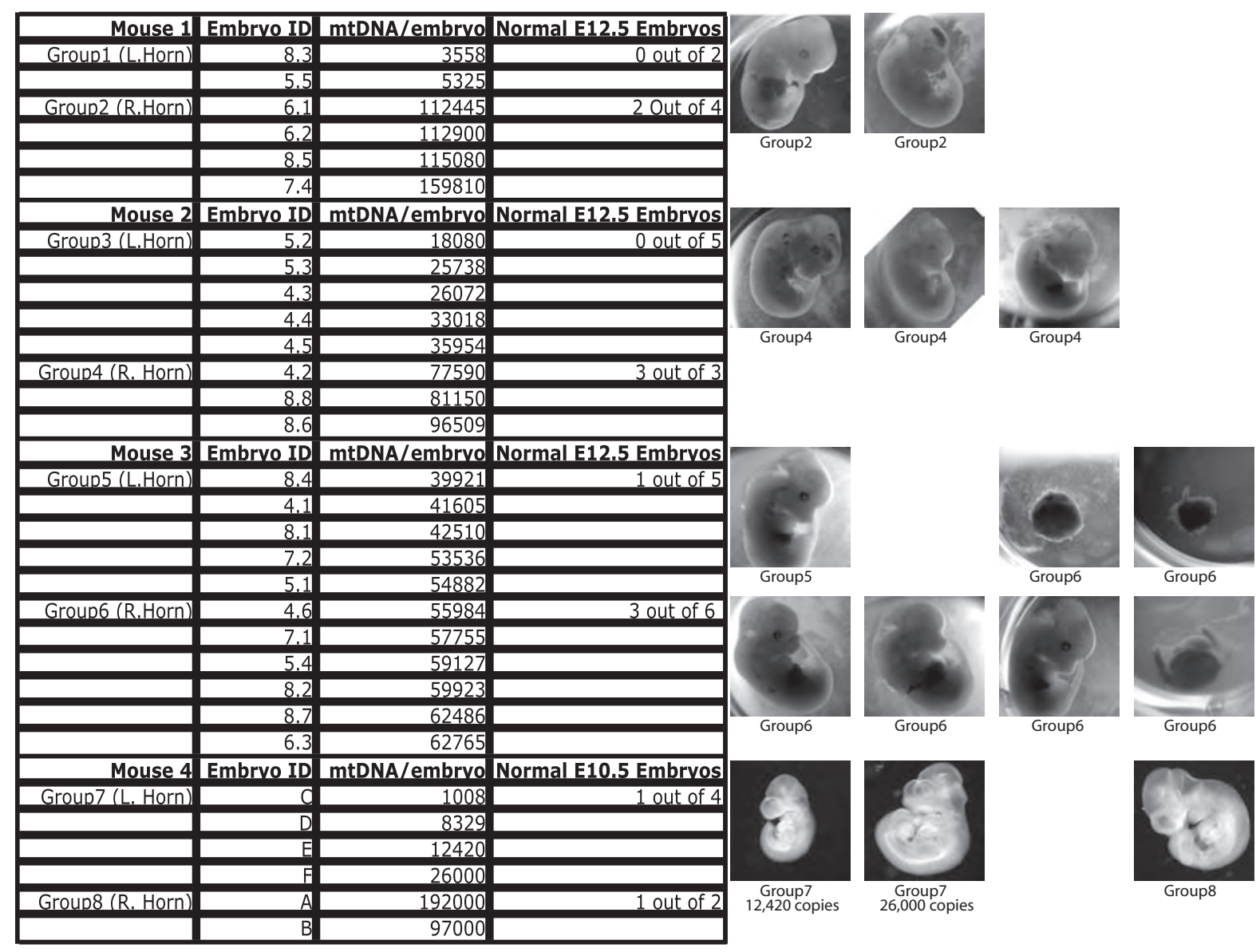


Figure 4

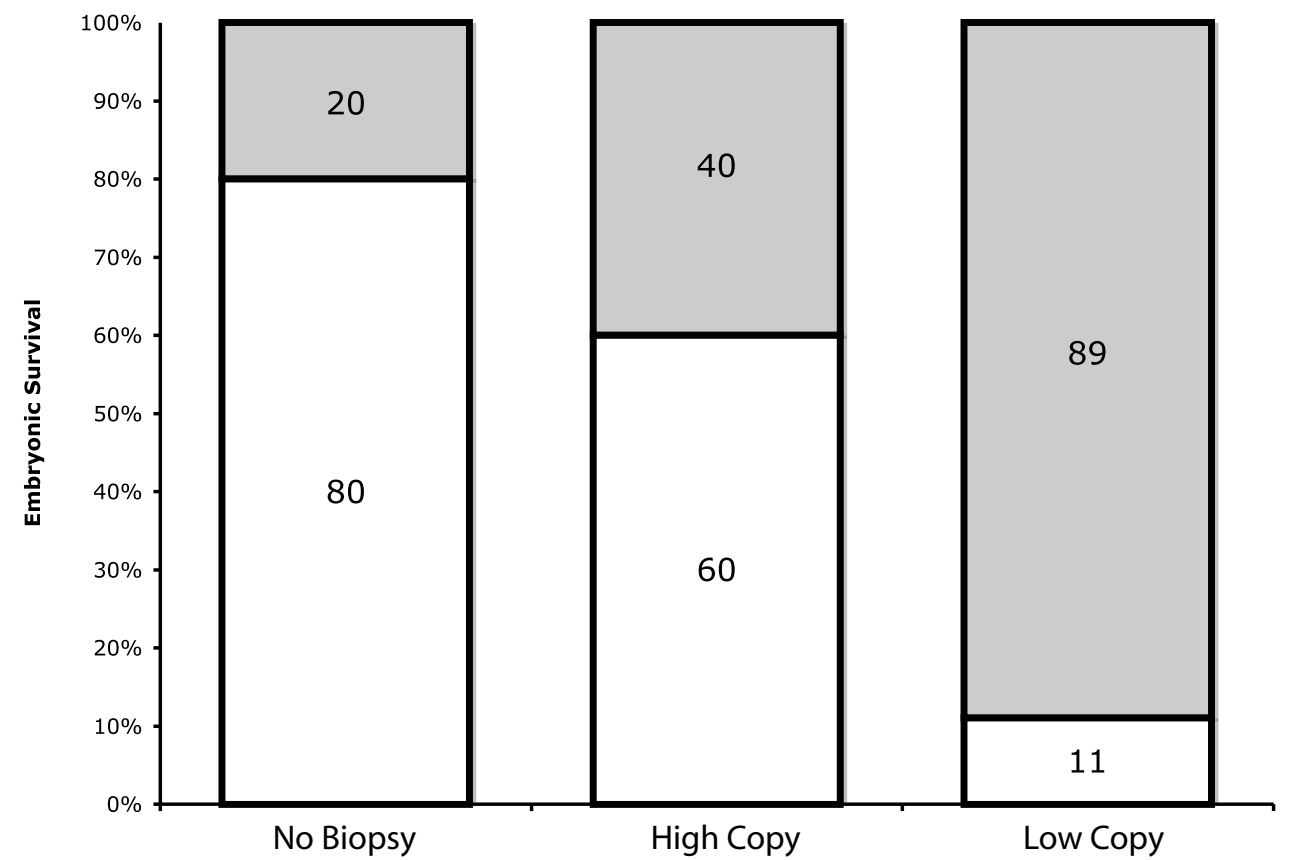




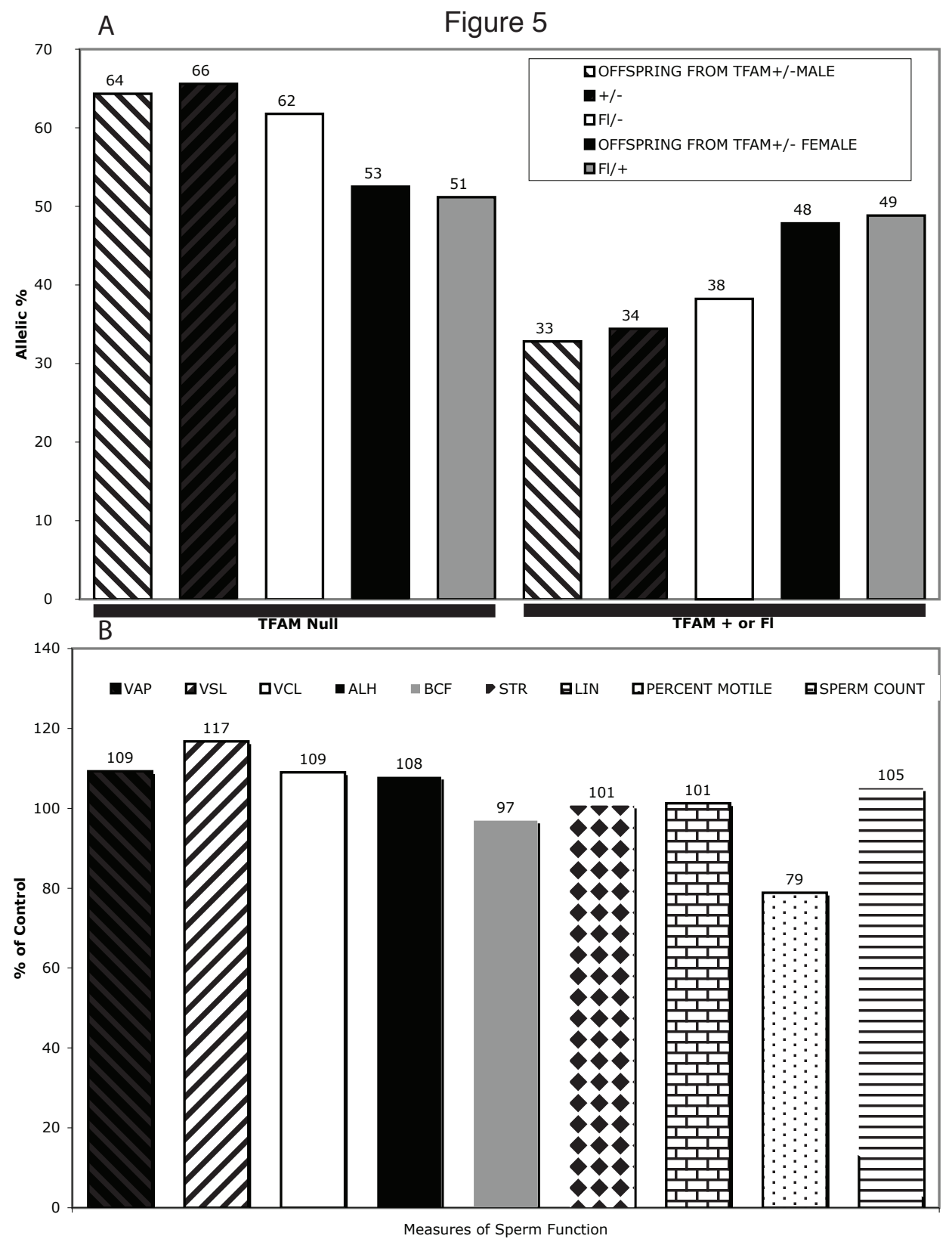


Figure 6

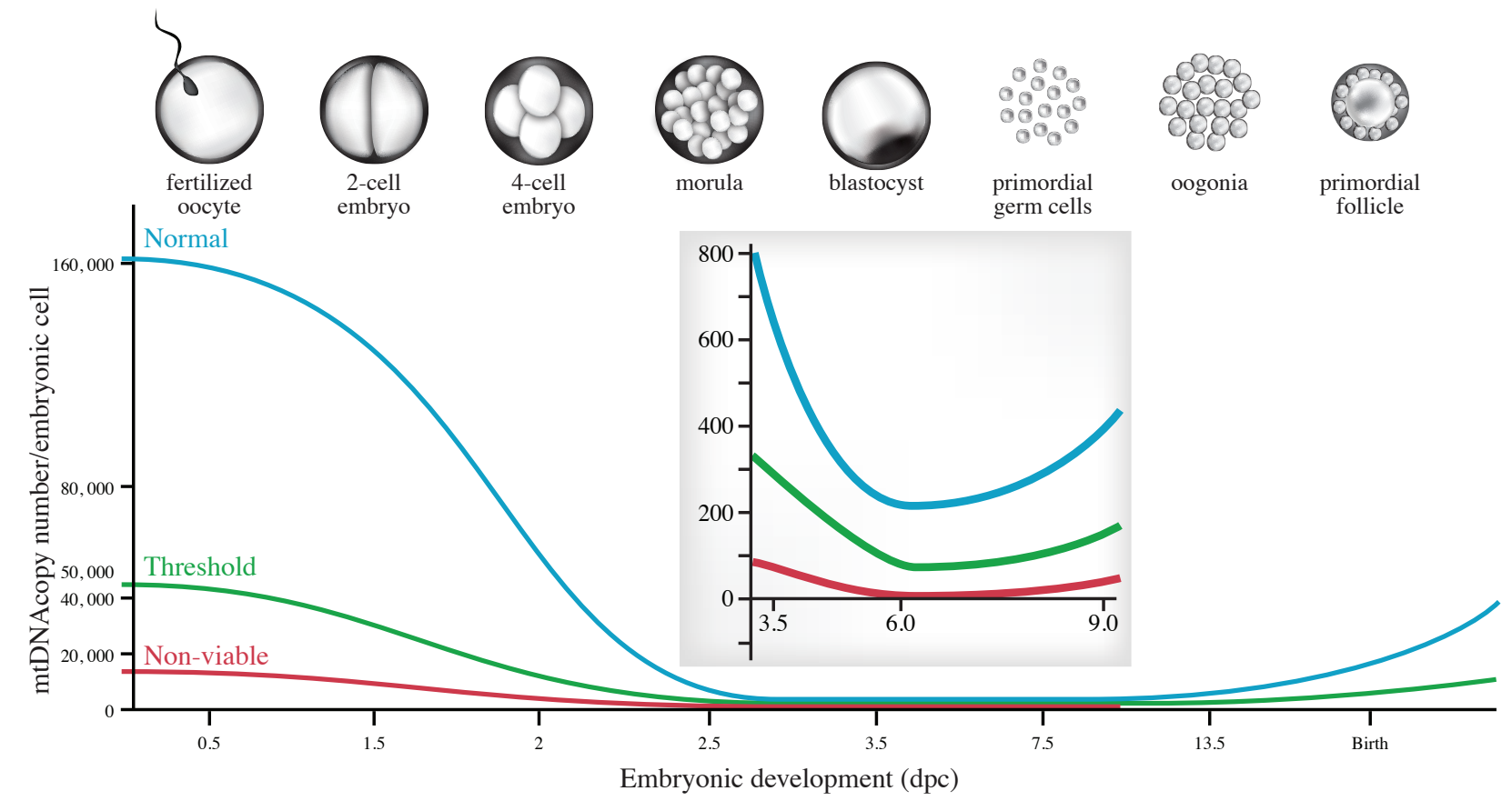




\section{General Discussion}

The purpose of my thesis was to explain the transmission of mitochondrial DNA in the female germline of the mouse. I analyzed the segregation of BALB and NZB mtDNA sequence variants and mtDNA copy number in the cells of the developing germline in order to establish a new working model for the mitochondrial DNA genetic bottleneck, which is presented in Chapter 1. Several lines of genetic and cell biological evidence that implicate a subgroup of replicating genomes as the mechanism responsible for the rapid segregation of mtDNA sequence variants in the germline of a heteroplasmic mouse model are presented. From these data, I adapted a population genetics equation ${ }^{142}$ to calculate the size of the mitochondrial genetic bottleneck, which accounts for the segregation of mtDNA in the germline of this mouse model. In Chapter 2, I show that early, but not late, germline depletion of TFAM in this heteroplasmic model leads to an increased rate of segregation due to a decreased nucleoid and mtDNA copy number in the oocyte. In addition to an unexpected ten-fold reduction in germline mtDNA copy number, late germline deletion of TFAM can also cause female infertility. I investigated the impact of mtDNA copy number in the germline in Chapter 3 and evaluated its importance to gametogenesis and embryogenesis.

The aim of Chapter 1 was to uncover the rules of transmission of mtDNA in the female germline. The rapid segregation of mtDNA sequence variants in heteroplasmic animal models and human pedigrees is due to an intergenerational mtDNA genetic bottleneck ${ }^{3}$, which was thought to immediately result from the 
creation of a physical mtDNA bottleneck in embryogenesis. At the time of specification, the germ cell precursors, termed primordial germ cells (PGCs), contain hundreds of mtDNAs. Since this would necessitate an expansion in both cell number and mtDNA copy number in order to regenerate the hundreds of thousands of copies of mtDNA present in mature oocyte, it seemed logical to propose that rapid segregation of mtDNA would occur during the first few mitotic divisions of the PGCs, when the pool of available mtDNA templates is smallest ${ }^{4}$.

In Chapter 1, I tracked the segregation of mtDNA in the female germline of a heteroplasmic mouse model by following the evolution of genotypic variance in the cells of the developing female germline. A previous post-doctoral fellow in the Shoubridge lab, Jack Jenuth, created this mouse model to study the germline segregation of $\mathrm{mtDNA}^{4}$ yet several important biological and statistical tools were lacking at the time of the original study, preventing a comprehensive and quantitative evaluation of mtDNA in the developing germline. Initially, the aims of my thesis were designed to be extensions his work.

Jenuth et al. undertook the manual isolation of histochemically identified PGCs, which limited the number of cells that were collected. They examined a handful of TNAP-positive PGCs along with a number of postnatal immature and mature oocytes and reported that the intercellular variation in heteroplasmy was much lower in the embryonic cells than in the post-natal cells. The authors advanced the hypothesis that the rapid segregation of mtDNA in the female germline was most likely taking place during the first few mitotic divisions of the PGCs. 
When beginning my work, I expected I would discover an increase in genotypic variance during early oogenesis. In order to more rapidly and easily isolate these embryonic cells we took advantage of an EGFP reporter transgene exhibiting strong and specific embryonic germ cell trans-activation from E8.5 to E14.5 (GOF18deltaPE-EGFP). The plan was to individually sort large numbers of embryonic germ cells by FACS and then determine mtDNA heteroplasmy and copy number by Real Time Q-PCR. Initial attempts at PGC isolation by FACS quickly revealed this method to be unreliable and inconsistent, forcing me to revert to manual isolation of these single cells.

The non-parametric analyses of genotypic variance in these cells failed to reveal rapid segregation of mtDNA during the mitotic proliferation of the PGCs. In an attempt to pinpoint the mitochondrial genetic bottleneck, Chinnery and coworkers used a computer program to model the expected rate of segregation based on measurements of mtDNA copy number they had made in the embryonic germline. These in silico predictions place the mitochondrial genetic bottleneck in early oogenesis. They postulated that $70 \%$ of the variance is generated by the time PGCs arise, even before mtDNA replication restarts and that most of the genotypic variance results from physical partitioning and sampling in the absence of mtDNA replication. This conclusion is not supported by the empirical measurements of germline heteroplasmy heteroplasmic variance presented in Chapter 1.

In Chapter 1, I ascribe the increase in genotypic variance in primary oocytes to the transition from primary to secondary follicles. The rapid 
segregation of mtDNA is most logically explained by the replication of a subgroup of templates, given the modest increase in mtDNA copy number between primary oocytes from primary and secondary follicle (13,000 copies to 26,000 copies). BrdU labeling of replicating mtDNA in the primary oocytes imaged by confocal and electron microscopy validated this hypothesis; however, the limited spatial resolution of confocal microscopy $(\sim 200 \mathrm{~nm})$ did not enable the accurate determination of either the number of replicating genomes or the structure of the sub-mitochondrial nucleoids in these cells. BrdU-labeling studies in the postnatal ovary also reveal that a proportion of cytoplasmic BrdU-positive punctae (which we interpret as replicating mtDNAs) display a lower degree of colocalization with markers of the nucleoid, such as TFAM and mt-SSB, than what is observed in postnatal somatic tissue sections. However, the highest degree of colocalization is seen in cultured C2C12 myoblast cells.

There are several possible interpretations of these observations. First, the absence of BrdU immunoreactivity in a TFAM-positive nucleoid puncta could be explained by the absence of replicating mtDNA, at least at the levels necessary for immunodetection with an anti-BrdU antibody. However, it is unlikely that the absence of TFAM co-immunoreactivity in BrdU-positive punctae represents naked molecules of replicating mtDNA. The mitochondrial genome requires supercoiling and compaction in order to fit and function within the confines of the mitochondrial matrix, so it is unlikely that the TFAM-negative BrdU-positive punctae actually reflect naked mtDNA. Rather, it is more plausible that the amount of TFAM present is below the immunodectable threshold: this represents 
a technical limitation that could theoretically extend to the immunological detection of any other protein component of the nucleoid. Nevertheless, the amount of immunodetectable TFAM in BrdU punctae may provide some clues as to the mechanism of replication of mtDNA. In vitro, the efficiency with which TFAM is able to bind and physically compact plasmid DNA (containing the same sequences found in mammalian mtDNA) has led to the hypothesis that TFAM virtually coats the entire mitochondrial genome, but general agreement regarding the stoichiometry of TFAM and mtDNA has yet to be acheived ${ }^{32,199-202}$. In such a tightly supercoiled state, it would be difficult for the transcription and replication machinery to gain access to the mtDNA. Therefore, the decompaction of the genome would require the release of (and hence local decrease in) TFAM protein from mtDNA in a manner similar to the decondensation of the nuclear DNA, which is regulated by the acetylation, methylation, and phosphorylation of certain amino acid residues of histones. While there is yet no compelling evidence that would suggest TFAM is subject to such post-translational modifications, it is tempting to invoke TFAM-mediated decompaction of mtDNA as a regulatory mechanism for replication and expression. The evidence of TFAM immunoreactivity colocalizing to mitochondria, imaged by both TEM and confocal microscopy, lends credence to the validity and specificity of the anti-TFAM antibody. While the colocalization of nucleoid markers and mtDNA in cultured cells is easily visualized by the incorporation of DNA-intercalating live dyes such as Pico Green ${ }^{203}$, there are no reports of such studies performed in vivo. It would be interesting to determine the degree of colocalization of these nucleoid markers 
and both replicating mtDNA (identified by BrdU incorporation) and quiescent mtDNA (visualized by FISH) with the aim of providing greater insight into the distribution and stochiometric requirements of TFAM in the process of mtDNA replication.

In practice, though, the spatial resolution limits of traditional confocal microscopes (which is $200 \mathrm{~nm}$ ) make it virtually impossible to resolve submitochondrial structures such as nucleoids. However, immunocytochemical study of the mitochondrial proteins and mtDNA would, in theory, be amenable to imaging with new generation imaging techniques such as $\mathrm{PALM}^{204}$ and STORM $^{205}$ that both report near-molecular resolution $(\sim 20 \mathrm{~nm})$ and which may be sufficient to determine the structure and organization of the nucleoid.

In the past, imaging of mtDNA and nucleoids by direct or indirect immunofluorescence confocal microscopy has been performed exclusively using cultured cells ${ }^{21,22,203}$. In light of the differing degrees of nucleoid markers and replicating mtDNA colocalization observed between cultured mouse cells and frozen sections of various mouse tissues I described in Chapter 1, it is important that we be cautious when extrapolating conclusions based on observations made in immortalized, potentially transformed (and thus highly aneuploid) cell lines to the biology of cells in vivo. While proper functioning of the enzyme complexes of the respiratory chain is an absolute requirement in virtually all mammalian cell types, examples of tissue-specific differences of nuclear-encoded mitochondrial proteins that are functionally indispensable in some tissues while less so in others implicate to functional redundancies and tissue-specific stochiometric 
requirements of mitochondrial proteins ${ }^{187,206,207}$. Adding another layer of complexity, the metabolic state of a tissue, cell, or organism could have a functional effect on the structure and composition of these multi-subunit complexes. In yeast, metabolic signals have been proposed to structurally alter the nucleoid ${ }^{26}$. Hence, we must acknowledge the possibility of such tissuespecificities and metabolic-sensitivities when designing experiments aimed at revealing the composition, structure, and function the mammalian nucleoid. If we are successful in unraveling the complexities of nucleoid dynamics, particularly as they relate to the signals that stimulate or repress the replication and expression of mtDNA, we may gain some more insight into the mechanism that selects the subgroup of replicating genomes in primary oocytes.

In the primary oocytes, the selection and amplification of these mtDNAs coincides with a transient and asymmetrical redistribution of mitochondria and mtDNA about a structure classically referred to as the Balbiani body $(\mathrm{Bb})$. While its existence has been known for more than 150 years ${ }^{126}$, the molecular function of this structure in mammals remains to be elucidated. Initially proposed by Spradling and coworkers to function in the inheritance of mitochondria in files, subsequent studies from the same group have called into question the relevance of the $\mathrm{Bb}^{208}$, whose transient presence in the developing murine oocyte made its definitive identification a difficult task ${ }^{127}$. Given its fleeting nature, we were excited to observe the co-occurrence of the $\mathrm{Bb}$ with the increase in genotypic variance in the post-natal oocyte. The asymmetrical aggregation of mitochondria about the $\mathrm{Bb}$ might have provided a simple explanation for template selection. 
However, it is not possible to invoke such a mechanism given the results presented in Chapter 1, wherein BrdU-labeling studies imaged with both immunohistochemistry and immuno-gold EM demonstrate BrdU-positive punctae in all regions of the primary oocyte, including in mitochondria situated furthest away from the $\mathrm{Bb}$. It should be noted that while numerous sections of primary oocytes were examined (oocytes progressing through different stages of folliculogenesis are captured in various regions of the same section) the resulting micrographs presented in Chapter 1 are merely a snapshot of the developing oocyte. It would be ideal to track the movements of mitochondria within individual primary oocytes in real time so to determine whether local mtDNA replication is exclusively initiated in mitochondria adjacent to the $\mathrm{Bb}$, which may later be displaced to more distant regions of the oocyte. Observing mitochondria via livecell imaging of primary oocytes from ovarian explants could be used to study the movement of mammalian mitochondria within primary oocytes.

Revealing the structure of the nucleoid and its relevance to mtDNA replication and expression, particularly in the selection of a subgroup of genomes designated for replication may provide us with a better understanding of the size of the mitochondrial genetic bottleneck. The current aforementioned limitations of confocal microscopy leave no choice but to evaluate the size of the mitochondrial genetic bottleneck (i.e. the number of segregating units) using alternative approaches.

In Chapter 2, I determined the size of the mitochondrial genetic bottleneck using a population genetics equation and then validated the working model for 
mtDNA segregation proposed in Chapter 1 by using germline-specific knockout mouse models that alter mtDNA copy number and OXPHOS function in the cells of the germline. The size of the mitochondrial genetic bottleneck in BALB heteroplasmic mice segregating BALB and NZB mtDNA was originally estimated to be about 185 segregating units ${ }^{4}$. Using the new derivation of the Sewell-Wright equation, I calculate that the mean bottleneck size in wild type heteroplasmic mice to between 135 and 189 segregating units depending upon the nuclear genetic background. The previous derivation, used by Jenuth et al, overestimates the bottleneck size by a factor of approximately 1.5 , relative to the new derivation I employed in the Chapter 2. Previously, the number of generations in the equation (represented by " $\mathrm{t}$ ") had been set to 15 to reflect the number of mitotic divisions of the germline. A new derivation is warranted particularly given that the mitochondrial genetic bottleneck occurs in the postmitotic female germ cell. This new derivation, which uses empirical measurements of mtDNA copy number and heteroplasmy variance to determine bottleneck size and the number of generations, makes no a priori assumptions regarding the percentage of replication genomes or the location of the mitochondrial genetic bottleneck. It differs from the original model insofar as it calculates the minimal number of mtDNA doublings, and not cell divisons, that would be required to generate the observed heteroplasmic variance and mtDNA copy number in the cells of developing germline. Since the size of the bottleneck is reduced by approximately $50 \%$ in female germlines from $\mathrm{TFAM}^{+-}$animals, which is explained by depletion of TFAM protein and nucleoid number in the 
developing post-natal ovary, the evidence in Chapter 2 also support the notion of the mitochondrial DNA nucleoid acting as the segregating unit in the germline of the mouse. It is important to remember, however, that the determination of bottleneck size (i.e number of segregating units) is a purely statistical calculation based on simplifying assumptions and should be validated using independent methodology. Nevertheless, the new derivation of the Sewell-Wright equation accounts for the observed mtDNA copy number and genotypic variance in the germline as well as the segregation pattern in heteroplasmic offspring.

While the segregation of neutral sequence variants has been instrumental in determining the mechanism and size of the mitochondrial genetic bottleneck, the next challenge will be to characterize the mechanism responsible segregation of pathogenic mtDNA mutations. The population incidence of mitochondrial diseases due to mutations in mtDNA is roughly 1 in 5000 and thus ranks among the most common types of inherited metabolic disorders ${ }^{209}$. Surprisingly, the occurrence of these mutations in the general population is far higher, with approximately 1 in 200 individuals carrying a pathogenic mtDNA mutation ${ }^{210}$. Until recently, selection against pathogenic mtDNA mutations in the germline was thought to be weak or non-existent as transmission in human pedigrees could be largely accounted for by random genetic drift ${ }^{4,75}$. However, relatively few mtDNA mutations are responsible for human mitochondrial diseases, and most occur in tRNA genes ${ }^{211}$. Using separate yet complementary approaches, the laboratories of Nils-Goran Larsson ${ }^{130}$ and Doug Wallace ${ }^{129}$ advanced an hypothesis for the existence of a germline filter for purifying selection, that would partly account for 
the observed discordance between pathogenic mutation frequency and disease incidence in human populations.

Wallace generated a mouse model carrying severe frameshift mutation in a gene coding for a subunit of complex I (ND6), which entirely abolished the enzymatic activity of the complex. While the mutation was first identified in homoplasmic state, Wallace and coworkers eventually isolated a functionally heteroplasmic line of embryonic stem cells, which contained the original ND6 mutation and a new mutation that restored the reading frame of the ND6 gene. They created chimeric animals from the injection of these cells into normal blastocysts and obtained a single founder female. Analyses of oocytes and offspring from this female showed that the frameshift mutation was selected against during oocyte maturation, suggesting that there is strong purifying selection in the germline for severe mtDNA mutations. I compared the reported genotypic variance in mature oocytes from this mouse model to the genotypic variance measured in wild-type oocytes and observed no significant differences, indicating that the selective elimination must be occurring before the early postnatal mitochondrial genetic bottleneck.

Stewart et al ${ }^{130}$ demonstrated the selective elimination of severe mtDNA mutations using a completely different strategy. They mated mice carrying a proof reading mutation in the gene coding for the mtDNA polymerase (POLG) with wildtype males to restore normal polymerase function, and tracked the evolution of mtDNA mutations that were caused by the defective polymerase in successive backcross generations. While mtDNA mutations were introduced randomly 
across the genome (with approximately 30 mutations per genome), the authors noted the conspicuous absence of non-synonymous mutations in protein-coding genes in the offspring of the second backcross generation. This bias supported the existence of a filter preventing the transmission of deleterious germline mtDNA mutations, but did not appear to select against either tRNA nor rRNA mutations in these animals.

These studies leave a number of key questions unanswered, the most important of which is what is the mechanism for purifying selection? Why do tRNA and rRNA mutations apparently escape purifying selection? Do mutations have to be severely deleterious to be subject to selection? How do some severe mutations in protein coding genes, such as those in ATP6 that are associated with early onset lethal human disease ${ }^{211}$, escape selection? Although mtDNA experiences a genetic bottleneck in the transmission from mother to offspring ${ }^{4}$, this cannot explain the bias against transmitting deleterious alleles. Fan et al suggest that selection could occur because the mutation mediates apoptosis of the developing oocytes (triggered by an increase in reactive oxygen species production), although the statistically identical genotypic variances measured in oocytes from Wallace's mutant mice and wild-type heteroplasmic mice do not support this hypothesis ${ }^{141}$. Moreover, the fact that very low proportions of the frameshifted ND6 gene, well below a mutant load that would elicit a cellular phenotype, are rapidly eliminated make it difficult to imagine how the filter for purifying selection could be acting at the cellular level. 
It seems much more likely that selection occurs at the level of the organelle itself. At least two mechanisms are possible. Mitochondria harboring mutant mtDNAs could be targeted for destruction by autophagy as there is some evidence that this process can be selective ${ }^{212}$. If this were the mechanism, functional impairment of germline "mitophagy" would be expected to lead to an increase in severe mutations in the next generation in mice carrying a defective mtDNA polymerase gene. Recently, a mouse deficient in mitochondrial autophagy in erythrocytes was identified ${ }^{213}$, although it remains to be determined whether the germline autophagy is affected in these mice.

Another mechanism would simply involve a relative selective advantage to wild-type mtDNAs during the mitochondrial expansion that occurs in oogenesis. MtDNA has a curious natural history during the development of the female germline. Mature oocytes contain nearly 200,000 copies of $\mathrm{mtDNA}^{6}$, thought to occur at 1-2 copies per organelle, which are apportioned without mtDNA replication to the cells of the early embryo. By the time mtDNA replication resumes, primordial germ cells contain approximately 100 mitochondria ${ }^{6}$.

The relatively low copy number of mtDNA per organelle in primordial germ cells might be important to expose the most severe mutations to selection. For mutations in protein coding genes the reduction in the amount of functional protein is directly proportional to the level of heteroplasmy, whereas the proportion of mutant tRNA genes typically must exceed $80 \%$ for expression of a biochemical phenotype ${ }^{211}$. Purifying selection could occur simply by making replication of mtDNA in the most dysfunctional mitochondria less efficient. This 
might for instance involve less efficient import of proteins necessary for mitochondrial biogenesis in organelles with a lowered membrane potential. The importance of starting such a process in early germline development is that selection can act on the multiple rounds of mtDNA replication, which are needed to regenerate the hundreds of thousands of copies of mtDNA present in the mature oocyte. Several lines of evidence presented in the final chapter of my thesis enable me to conclude that the large number of mtDNAs in the mature oocyte in fact plays a functional role in postimplantation development of the embryo.

In Chapter 3, I presented several lines of evidence to support the existence of a threshold of mtDNA important in the development of the embryo. The studies examining the role of mtDNA copy number in gametogenesis initially began with the observations I made when I was having difficulty successfully breeding heteroplasmic TFAM ${ }^{\mathrm{fl} /+} ; \mathrm{Zp} 3-\mathrm{Cre}^{+}$females. When it became clear that mtDNA copy number in oocytes from these animals was markedly reduced, experiments were designed to directly test the critical copy number requirement for embryogenesis. While the number of embryos examined in this study was not as great as in Chapter 1 and 2, the significant differences in the rates of postimplantation that exist between low mtDNA copy number and high mtDNA copy number $\mathrm{TFAM}^{+-}$embryos point to a developmentally crucial threshold of approximately 50,000 copies of mtDNA. But if 50,000 copies of mtDNA define an absolute developmental threshold, how might one reconcile the seemingly normal developmental progression followed by embryos with fewer than 50,000 copies 
(e.g. Mouse 4, Blastomere F)? Before outlining plausible explanations, I will first examine the results of the transfer experiment that appear to directly contradict the notion of a 50,000 mtDNA copy number threshold for development. In this example, the Low Copy group embryos $(n=4)$ transferred to Mouse 4 originated from different $\mathrm{TFAM}^{+/-}$mothers and had different enough heteroplasmy levels that the surviving E10.5 embryos could be traced back to the blastomere that was used estimate mtDNA copy number. Of these 4 transferred embryos (which contained some of the lowest mtDNA copy number embryos of all), only the embryos with the highest mtDNA copy number (Blastomere E and F) survived with the lower of the two exhibiting severe developmental delay. While the seemingly normal development of the remaining E10.5 embryo contradicts the proposed strict developmental threshold, it is worthwhile to note that a fertilized oocyte carrying 26,000 mtDNAs still carries $\sim 1.5$ times more mtDNA than the mean fertilized oocyte from the largely infertile $\operatorname{TFAM}^{\mathrm{fl} /+} ; \mathrm{Zp}^{+}$females. The postimplantation lethality that manifests in embryos derived from oocytes from $\operatorname{TFAM}^{\mathrm{fl}+} ; \mathrm{Zp} 3$ females (which contain about 18,000 copies) may be caused by mitochondrial insufficiency. Yet, these cells would be predicted to contain only a couple dozen copies of mtDNA per embryonic germ cell by the time mtDNA replication restarts (at E6.0), assuming equal distribution of organelles into the cells of the developing embryo. The lethality in these embryos may stem from defects in energy metabolism but could also reflect defects in other mitochondrial processes such as calcium homeostasis or apoptosis. 
While the precise signals and mechanism that restart mtDNA replication in the postimplantation embryo have yet to be uncovered, it is not impossible that postimplantation embryos resulting from the fertilization of oocytes carrying fewer than the 50,000 copies upregulate mitochondrial biogenesis or initiate mtDNA replication earlier in development as a cellular compensatory response (e.g. mtDNA upregulation in the germline of $\mathrm{COX} 10^{+/-}$females). Such variability in mtDNA replication may already exist under normal conditions in wild-type embryos and has been proposed to contribute to the biological variability of mtDNA copy number in $\mathrm{PGCs}^{5}$, which could reflect the cellular demands for energy production. However it is achieved, variability in the resumption of mitochondrial biogenesis and mtDNA replication could also impact the variability in embryonic lethality observed in homozygous knockout mouse models for some nuclear-encoded mitochondrial genes (Table 1).

In this thesis I describe several novel contributions to our understanding regarding the transmission of mitochondrial DNA in the female germline of the mouse and in so doing raise new questions regarding the evolutionary impetus driving the separation of the mitochondrial genetic and physical bottlenecks. If the mechanism responsible for the selective elimination of severe mutations in mtDNA relies on a reduction in mtDNA copy number per organelle and multiple rounds of mtDNA replication in order to expose and effectively eliminate severe mutations, the elevated levels of mtDNA copy number in the mature oocyte may simply reflect an evolutionary compromise: the oocyte levels of mtDNA are such that a sufficient amount of mtDNA are apportioned to the cells of the embryo, yet 
low enough in the primordial germ cells to provide the filter for purifying selection with a sufficient number of rounds of mtDNA replication over which to selectively and progressively eliminate severe mtDNA mutations. In addition to uncovering the functional and molecular basis of selection, it will be of great interest to determine if the efficiency of this filter is contingent upon mtDNA copy number in the PGCs. Indeed, a clear understanding of this mechanism will be necessary if we are to develop strategies aimed at preventing the transmission of pathogenic mtDNA mutations that, in humans, can cause a variety of multi-systemic disorders with no known cures. 


\begin{tabular}{|c|c|c|c|}
\hline Gene Name & Function & Embryonic Lethality & Study \\
\hline ACC1 & Fatty Acid Metabolism (acetyl-CoA to malonyl-CoA) & E8.5 & Abu-Elheiga et al., 2005 \\
\hline AIF1 & Apoptosis & E9 & Brown et al., 2006 \\
\hline APAF1 & Apoptosis & E16.5 & Cecconi et al., 1998 \\
\hline $\operatorname{cox} 10$ & OXHPOS (COX Assembly Factor) & E10.5 & This study \\
\hline COX17 & OXHPOS (COX Assembly Factor) & E8.5-10 & Takashi et al., 2002 \\
\hline CPT1B & Beta-oxidation & E9.5-11.5 & Ji et al., 2008 \\
\hline CYTC & OXPHOS (COX) & E8.5 & Li et al., 2000 \\
\hline ENGOG & Mitochondrial DNA Replication and Nuclear Fragmentation During Apoptosis & E2.5-E3.5 & Zhang et al., 2003 \\
\hline FRDA & Mitochondrial Iron Homeostasis & E7.5-E9.5 & Cossee et al., 2000 \\
\hline HD & Huntington's Disease & E8.5 & Nasir et al., 1995 \\
\hline MFN1 & Mitochondrial Dynamics & E12.5 & Chen et al., \\
\hline NMDMC & Mitochondrial Folate Metabolism & E12.5 & Di Pietro et al., 2002 \\
\hline$\overline{P O L G A}$ & Mitochondrial DNA Replication & E7.5 -E8.5 & Hance et al., 2005 \\
\hline RNASEH1 & Mitochondrial DNA Maintenance & E8.5 & Cerritelli et al., 2003 \\
\hline SLC25A19 & Mitochondrial deoxyribonucleotide transporter & E12 & Lindhurst et al., 2006 \\
\hline SURF1 & OXPHOS (COX Assembly Factor) & E6.5 to E12 & Agostino et al., 2003 \\
\hline TFAM & Mitochondrial DNA Maintenance, Replication, Expression & E8.5-E10.5 & Larsson et al., 1998 \\
\hline
\end{tabular}




\section{References:}

1. Bogenhagen, D. \& Clayton, D.A. Mouse $L$ cell mitochondrial DNA molecules are selected randomly for replication throughout the cell cycle. Cell 11, 719-27 (1977).

2. Shoubridge, E.A. \& Wai, T. Mitochondrial DNA and the mammalian oocyte. Curr Top Dev Biol 77, 87-111 (2007).

3. Hauswirth, W.W. \& Laipis, P.J. Mitochondrial DNA polymorphism in a maternal lineage of Holstein cows. Proc Natl Acad Sci U S A 79, 4686-90. (1982).

4. Jenuth, J.P., Peterson, A.C., Fu, K. \& Shoubridge, E.A. Random genetic drift in the female germline explains the rapid segregation of mammalian mitochondrial DNA [see comments]. Nat Genet 14, 146-51 (1996).

5. Cree, L.M. et al. A reduction of mitochondrial DNA molecules during embryogenesis explains the rapid segregation of genotypes. Nat Genet 40, 249-54 (2008).

6. Cao, L. et al. The mitochondrial bottleneck occurs without reduction of mtDNA content in female mouse germ cells. Nat Genet 39, 386-90 (2007).

7. Clayton, D.A. Replication and transcription of vertebrate mitochondrial DNA. Annu Rev Cell Biol 7, 453-78 (1991).

8. Shadel, G.S. \& Clayton, D.A. Mitochondrial DNA maintenance in vertebrates. Annu Rev Biochem 66, 409-35 (1997).

9. Yang, M.Y. et al. Biased incorporation of ribonucleotides on the mitochondrial L-strand accounts for apparent strand-asymmetric DNA replication. Cell 111, 495-505 (2002).

10. Holt, I.J., Lorimer, H.E. \& Jacobs, H.T. Coupled leading- and laggingstrand synthesis of mammalian mitochondrial DNA. Cell 100, 515-24. (2000).

11. Bowmaker, M. et al. Mammalian mitochondrial DNA replicates bidirectionally from an initiation zone. J Biol Chem 278, 50961-9 (2003).

12. Brown, T.A., Cecconi, C., Tkachuk, A.N., Bustamante, C. \& Clayton, D.A. Replication of mitochondrial DNA occurs by strand displacement with alternative light-strand origins, not via a strand-coupled mechanism. Genes Dev 19, 2466-76 (2005).

13. Brown, T.A. \& Clayton, D.A. Genesis and wanderings: origins and migrations in asymmetrically replicating mitochondrial DNA. Cell Cycle 5, 917-21 (2006).

14. Yasukawa, T. et al. Replication of vertebrate mitochondrial DNA entails transient ribonucleotide incorporation throughout the lagging strand. EMBO J 25, 5358-71 (2006).

15. Ebert, K.M., Liem, H. \& Hecht, N.B. Mitochondrial DNA in the mouse preimplantation embryo. J Reprod Fertil 82, 145-9 (1988).

16. Carrodeguas, J.A., Kobayashi, R., Lim, S.E., Copeland, W.C. \& Bogenhagen, D.F. The accessory subunit of Xenopus laevis mitochondrial DNA polymerase gamma increases processivity of the catalytic subunit of 
human DNA polymerase gamma and is related to class II aminoacyl-tRNA synthetases. Mol Cell Biol 19, 4039-46 (1999).

17. Fan, L., Sanschagrin, P.C., Kaguni, L.S. \& Kuhn, L.A. The accessory subunit of mtDNA polymerase shares structural homology with aminoacyltRNA synthetases: implications for a dual role as a primer recognition factor and processivity clamp. Proc Natl Acad Sci U S A 96, 9527-32 (1999).

18. Wong, T.W. \& Clayton, D.A. Isolation and characterization of a DNA primase from human mitochondria. J Biol Chem 260, 11530-5 (1985).

19. Shutt, T.E. \& Gray, M.W. Twinkle, the mitochondrial replicative DNA helicase, is widespread in the eukaryotic radiation and may also be the mitochondrial DNA primase in most eukaryotes. $J$ Mol Evol 62, 588-99 (2006).

20. Satoh, M. \& Kuroiwa, T. Organization of multiple nucleoids and DNA molecules in mitochondria of a human cell. Exp Cell Res 196, 137-40 (1991).

21. Legros, F., Malka, F., Frachon, P., Lombes, A. \& Rojo, M. Organization and dynamics of human mitochondrial DNA. J Cell Sci 117, 2653-62 (2004).

22. Gilkerson, R.W., Schon, E.A., Hernandez, E. \& Davidson, M.M. Mitochondrial nucleoids maintain genetic autonomy but allow for functional complementation. J Cell Biol 181, 1117-28 (2008).

23. Piko, L. \& Taylor, K.D. Amounts of mitochondrial DNA and abundance of some mitochondrial gene transcripts in early mouse embryos. Dev Biol 123, 364-74 (1987).

24. Hecht, N.B., Liem, H., Kleene, K.C., Distel, R.J. \& Ho, S.M. Maternal inheritance of the mouse mitochondrial genome is not mediated by a loss or gross alteration of the paternal mitochondrial DNA or by methylation of the oocyte mitochondrial DNA. Dev Biol 102, 452-61. (1984).

25. Kaufman, B.A. et al. In organello formaldehyde crosslinking of proteins to mtDNA: identification of bifunctional proteins. Proc Natl Acad Sci U S A 97, 7772-7 (2000).

26. Chen, X.J., Wang, X., Kaufman, B.A. \& Butow, R.A. Aconitase couples metabolic regulation to mitochondrial DNA maintenance. Science $\mathbf{3 0 7}$, 714-7 (2005).

27. Bogenhagen, D.F., Wang, Y., Shen, E.L. \& Kobayashi, R. Protein components of mitochondrial DNA nucleoids in higher eukaryotes. Mol Cell Proteomics (2003).

28. Bogenhagen, D.F., Rousseau, D. \& Burke, S. The layered structure of human mitochondrial DNA nucleoids. J Biol Chem 283, 3665-75 (2008).

29. Garrido, N. et al. Composition and dynamics of human mitochondrial nucleoids. Mol Biol Cell 14, 1583-96 (2003).

30. Holt, I.J. et al. Mammalian mitochondrial nucleoids: organizing an independently minded genome. Mitochondrion 7, 311-21 (2007). 
31. Alam, T.I. et al. Human mitochondrial DNA is packaged with TFAM. Nucleic Acids Res 31, 1640-5 (2003).

32. Kaufman, B.A. et al. The mitochondrial transcription factor TFAM coordinates the assembly of multiple DNA molecules into nucleoid-like structures. Mol Biol Cell 18, 3225-36 (2007).

33. Ekstrand, M.I. et al. Mitochondrial transcription factor A regulates mtDNA copy number in mammals. Hum Mol Genet 13, 935-44 (2004).

34. Davis, A.F. \& Clayton, D.A. In situ localization of mitochondrial DNA replication in intact mammalian cells. J Cell Biol 135, 883-93 (1996).

35. Birky, C.W., Jr. The Inheritance of genes in mitochodria and chloroplasts: Laws, Mechanisms, and Models. Annu Rev Genet 35, 125-48 (2001).

36. Gross, N.J., Getz, G.S. \& Rabinowitz, M. Apparent turnover of mitochondrial deoxyribonucleic acid and mitochondrial phospholipids in the tissues of the rat. J Biol Chem 244, 1552-62 (1969).

37. Fernandez-Silva, P., Martinez-Azorin, F., Micol, V. \& Attardi, G. The human mitochondrial transcription termination factor (mTERF) is a multizipper protein but binds to DNA as a monomer, with evidence pointing to intramolecular leucine zipper interactions. Embo J 16, 1066-79. (1997).

38. Martin, M., Cho, J., Cesare, A.J., Griffith, J.D. \& Attardi, G. Termination factor-mediated DNA loop between termination and initiation sites drives mitochondrial rRNA synthesis. Cell 123, 1227-40 (2005).

39. Walberg, M.W. \& Clayton, D.A. In vitro transcription of human mitochondrial DNA. Identification of specific light strand transcripts from the displacement loop region. J Biol Chem 258, 1268-75 (1983).

40. Bonawitz, N.D., Clayton, D.A. \& Shadel, G.S. Initiation and beyond: multiple functions of the human mitochondrial transcription machinery. Mol Cell 24, 813-25 (2006).

41. Falkenberg, M. et al. Mitochondrial transcription factors B1 and B2 activate transcription of human mtDNA. Nat Genet 31, 289-94 (2002).

42. Gaspari, M., Larsson, N.G. \& Gustafsson, C.M. The transcription machinery in mammalian mitochondria. Biochim Biophys Acta 1659, 14852 (2004).

43. Ojala, D., Montoya, J. \& Attardi, G. tRNA punctuation model of RNA processing in human mitochondria. Nature 290, 470-4 (1981).

44. Linder, $\mathrm{T}$. et al. A family of putative transcription termination factors shared amongst metazoans and plants. Curr Genet 48, 265-9 (2005).

45. Park, C.B. et al. MTERF3 is a negative regulator of mammalian mtDNA transcription. Cell 130, 273-85 (2007).

46. Roberti, M. et al. MTERF3, the most conserved member of the mTERFfamily, is a modular factor involved in mitochondrial protein synthesis. Biochim Biophys Acta 1757, 1199-206 (2006).

47. Spremulli, L.L., Coursey, A., Navratil, T. \& Hunter, S.E. Initiation and elongation factors in mammalian mitochondrial protein biosynthesis. Prog Nucleic Acid Res Mol Biol 77, 211-61 (2004). 
48. O'Brien, T.W. Evolution of a protein-rich mitochondrial ribosome: implications for human genetic disease. Gene 286, 73-9 (2002).

49. Chiquoine, A.D. The identification, origin, and migration of the primordial germ cells in the mouse embryo. Anat Rec 118, 135-46 (1954).

50. Ginsburg, M., Snow, M.H. \& McLaren, A. Primordial germ cells in the mouse embryo during gastrulation. Development 110, 521-8 (1990).

51. Saitou, M., Barton, S.C. \& Surani, M.A. A molecular programme for the specification of germ cell fate in mice. Nature 418, 293-300 (2002).

52. Tanaka, S.S. \& Matsui, Y. Developmentally regulated expression of mil-1 and mil-2, mouse interferon-induced transmembrane protein like genes, during formation and differentiation of primordial germ cells. Mech Dev 119 Suppl 1, S261-7 (2002).

53. Tanaka, S.S., Yamaguchi, Y.L., Tsoi, B., Lickert, H. \& Tam, P.P. IFITM/Mil/fragilis family proteins IFITM1 and IFITM3 play distinct roles in mouse primordial germ cell homing and repulsion. Dev Cell 9, 745-56 (2005).

54. Lawson, K.A. et al. Bmp4 is required for the generation of primordial germ cells in the mouse embryo. Genes Dev 13, 424-36 (1999).

55. Ying, Y., Qi, X. \& Zhao, G.Q. Induction of primordial germ cells from murine epiblasts by synergistic action of BMP4 and BMP8B signaling pathways. Proc Natl Acad Sci U S A 98, 7858-62 (2001).

56. Yoshimizu, T. et al. Germline-specific expression of the Oct-4/green fluorescent protein (GFP) transgene in mice. Dev Growth Differ 41, 675-84 (1999).

57. Szabo, P.E., Hubner, K., Scholer, H. \& Mann, J.R. Allele-specific expression of imprinted genes in mouse migratory primordial germ cells. Mech Dev 115, 157-60 (2002).

58. Tam, P.P. \& Snow, M.H. Proliferation and migration of primordial germ cells during compensatory growth in mouse embryos. J Embryol Exp Morphol 64, 133-47 (1981).

59. Molyneaux, K.A., Stallock, J., Schaible, K. \& Wylie, C. Time-lapse analysis of living mouse germ cell migration. Dev Biol 240, 488-98 (2001).

60. Buehr, $M$. The primordial germ cells of mammals: some current perspectives. Exp Cell Res 232, 194-207 (1997).

61. Fox, N., Damjanov, I., Martinez-Hernandez, A., Knowles, B.B. \& Solter, D. Immunohistochemical localization of the early embryonic antigen (SSEA-1) in postimplantation mouse embryos and fetal and adult tissues. Dev Biol 83, 391-8 (1981).

62. Wartenberg, H., Hilscher, B. \& Hilscher, W. Germ cell kinetics during early ovarian differentiation: an analysis of the oogonial cell cycle and the subsequent changes in oocyte development during the onset of meiosis in the rat. Microsc Res Tech 40, 377-97 (1998).

63. Godin, I., Wylie, C. \& Heasman, J. Genital ridges exert long-range effects on mouse primordial germ cell numbers and direction of migration in culture. Development 108, 357-63 (1990). 
64. Johnson, J., Canning, J., Kanedo, T., Pru, J.K. \& Tilly, J.L. Germline Stem Cells and Follicular Renewal in the Postnatal Mammalian Ovary. Obstet Gynecol Surv 59, 518-520 (2004).

65. Gosden, R.G. Germline stem cells in the postnatal ovary: is the ovary more like a testis? Hum Reprod Update 10, 193-5 (2004).

66. Jansen, R.P. \& de Boer, K. The bottleneck: mitochondrial imperatives in oogenesis and ovarian follicular fate. Mol Cell Endocrinol 145, 81-8. (1998).

67. Snow, M.H. Gastrulation in the mouse: Growth and regionalization of the epiblast. J Embryol Exp Morphol 42, 293-303 (1977).

68. Jansen, R.P. Germline passage of mitochondria: quantitative considerations and possible embryological sequelae. Hum Reprod 15

Suppl 2, 112-28. (2000).

69. Mulnard, J.G. [Microcinematographic analysis of the mouse egg development from stage 2 to the blastocyst]. Arch Biol (Liege) 78, 107-39 (1967).

70. Mulnard, J.G. [The phagocytic proterties of the trophoblast during the first phases of ovo-implantation in mice]. Arch Biol (Liege) 78, 575-94 (1967).

71. Barlow, P.W. \& Sherman, M.I. The biochemistry of differentiation of mouse trophoblast: studies on polyploidy. J Embryol Exp Morphol 27, 447-65 (1972).

72. Strickland, S., Reich, E. \& Sherman, M.I. Plasminogen activator in early embryogenesis: enzyme production by trophoblast and parietal endoderm. Cell 9, 231-40 (1976).

73. Brenner, C.A., Adler, R.R., Rappolee, D.A., Pedersen, R.A. \& Werb, Z. Genes for extracellular-matrix-degrading metalloproteinases and their inhibitor, TIMP, are expressed during early mammalian development. Genes Dev 3, 848-59 (1989).

74. Koehler, C.M. et al. Replacement of bovine mitochondrial DNA by a sequence variant within one generation. Genetics 129, 247-55 (1991).

75. Chinnery, P.F. et al. The inheritance of mitochondrial DNA heteroplasmy: random drift, selection or both? Trends Genet 16, 500-5. (2000).

76. Thundathil, J., Filion, F. \& Smith, L.C. Molecular control of mitochondrial function in preimplantation mouse embryos. Mol Reprod Dev 71, 405-13 (2005).

77. McConnell, J.M. \& Petrie, L. Mitochondrial DNA turnover occurs during preimplantation development and can be modulated by environmental factors. Reprod Biomed Online 9, 418-24 (2004).

78. Marchington, D.R., Hartshorne, G.M., Barlow, D. \& Poulton, J. Homopolymeric tract heteroplasmy in mtDNA from tissues and single oocytes: support for a genetic bottleneck. Am J Hum Genet 60, 408-16. (1997).

79. Brown, D.T., Samuels, D.C., Michael, E.M., Turnbull, D.M. \& Chinnery, P.F. Random genetic drift determines the level of mutant mtDNA in human primary oocytes. Am J Hum Genet 68, 533-6. (2001). 
80. Blok, R.B., Gook, D.A., Thorburn, D.R. \& Dahl, H.H. Skewed segregation of the mtDNA nt 8993 (T-->G) mutation in human oocytes. Am J Hum Genet 60, 1495-501 (1997).

81. Steffann, J. et al. Analysis of mtDNA variant segregation during early human embryonic development: a tool for successful NARP preimplantation diagnosis. J Med Genet 43, 244-7 (2006).

82. White, S.L. et al. Mitochondrial DNA mutations at nucleotide 8993 show a lack of tissue- or age-related variation. J Inherit Metab Dis 22, 899-914 (1999).

83. Chinnery, P.F. et al. The epidemiology of pathogenic mitochondrial DNA mutations. Ann Neurol 48, 188-93. (2000).

84. Inoue, K. et al. Generation of mice with mitochondrial dysfunction by introducing mouse mtDNA carrying a deletion into zygotes. Nat Genet 26, 176-81. (2000).

85. DiMauro, S. \& Schon, E.A. Mitochondrial respiratory-chain diseases. $N$ Engl J Med 348, 2656-68 (2003).

86. Chinnery, P.F. et al. Risk of developing a mitochondrial DNA deletion disorder. Lancet 364, 592-6 (2004).

87. Perez, G.I., Trbovich, A.M., Gosden, R.G. \& Tilly, J.L. Mitochondria and the death of oocytes. Nature 403, 500-1. (2000).

88. Krakauer, D.C. \& Mira, A. Mitochondria and germ-cell death. Nature 400, 125-6. (1999).

89. Boulet, L., Karpati, G. \& Shoubridge, E.A. Distribution and threshold expression of the tRNA(Lys) mutation in skeletal muscle of patients with myoclonic epilepsy and ragged-red fibers (MERRF). Am J Hum Genet 51, 1187-200 (1992).

90. Macmillan, C., Lach, B. \& Shoubridge, E.A. Variable distribution of mutant mitochondrial DNAs (tRNA(Leu[3243])) in tissues of symptomatic relatives with MELAS: the role of mitotic segregation. Neurology 43, 1586-90 (1993).

91. Schon, E.A. et al. A direct repeat is a hotspot for large-scale deletion of human mitochondrial DNA. Science 244, 346-9. (1989).

92. Keefe, D.L., Niven-Fairchild, T., Powell, S. \& Buradagunta, S. Mitochondrial deoxyribonucleic acid deletions in oocytes and reproductive aging in women. Fertil Steril 64, 577-83 (1995).

93. Brenner, C.A. et al. Mitochondrial DNA deletion in human oocytes and embryos. Mol Hum Reprod 4, 887-92 (1998).

94. Barritt, J.A., Brenner, C.A., Cohen, J. \& Matt, D.W. Mitochondrial DNA rearrangements in human oocytes and embryos. Mol Hum Reprod 5, 92733 (1999).

95. Hsieh, R.H. et al. Multiple rearrangements of mitochondrial DNA in unfertilized human oocytes. Fertil Steril 77, 1012-7 (2002).

96. Chan, C.C. et al. Mitochondrial DNA content and 4977 bp deletion in unfertilized oocytes. Mol Hum Reprod 11, 843-6 (2005). 
97. Steuerwald, N. et al. Quantification of mtDNA in single oocytes, polar bodies and subcellular components by real-time rapid cycle fluorescence monitored PCR. Zygote 8, 209-15 (2000).

98. Reynier, P. et al. Mitochondrial DNA content affects the fertilizability of human oocytes. Mol Hum Reprod 7, 425-9 (2001).

99. Barritt, J.A., Kokot, M., Cohen, J., Steuerwald, N. \& Brenner, C.A. Quantification of human ooplasmic mitochondria. Reprod Biomed Online 4, 243-7 (2002).

100. May-Panloup, P. et al. Low oocyte mitochondrial DNA content in ovarian insufficiency. Hum Reprod 20, 593-7 (2005).

101. Michaels, G.S., Hauswirth, W.W. \& Laipis, P.J. Mitochondrial DNA copy number in bovine oocytes and somatic cells. Dev Biol 94, 246-51 (1982).

102. Birky, C.W., Jr. Uniparental inheritance of mitochondrial and chloroplast genes: mechanisms and evolution. Proc Natl Acad Sci U S A 92, 11331-8 (1995).

103. Hurst, L.D., Atlan, A. \& Bengtsson, B.O. Genetic conflicts. Q Rev Biol 71, 317-64. (1996).

104. Sutovsky, P. et al. Ubiquitin tag for sperm mitochondria. Nature 402, 3712. (1999).

105. Gyllensten, U., Wharton, D., Josefsson, A. \& Wilson, A.C. Paternal inheritance of mitochondrial DNA in mice see comments]. Nature 352, 255-7 (1991).

106. Kaneda, $\mathrm{H}$. et al. Elimination of paternal mitochondrial DNA in intraspecific crosses during early mouse embryogenesis. Proc Natl Acad Sci U S A 92, 4542-6. (1995).

107. Shitara, H., Hayashi, J.I., Takahama, S., Kaneda, H. \& Yonekawa, H. Maternal inheritance of mouse mtDNA in interspecific hybrids: segregation of the leaked paternal mtDNA followed by the prevention of subsequent paternal leakage. Genetics 148, 851-7. (1998).

108. Zhao, $X$. et al. Further evidence for paternal inheritance of mitochondrial DNA in the sheep (Ovis aries). Heredity 93, 399-403 (2004).

109. Gur, Y. \& Breitbart, H. Mammalian sperm translate nuclear-encoded proteins by mitochondrial-type ribosomes. Genes Dev 20, 411-6 (2006).

110. Sutovsky, P. Ubiquitin-dependent proteolysis in mammalian spermatogenesis, fertilization, and sperm quality control: killing three birds with one stone. Microsc Res Tech 61, 88-102 (2003).

111. Sutovsky, P. et al. Ubiquitinated sperm mitochondria, selective proteolysis, and the regulation of mitochondrial inheritance in mammalian embryos. Biol Reprod 63, 582-90. (2000).

112. Nishimura, Y. et al. Active digestion of sperm mitochondrial DNA in single living sperm revealed by optical tweezers. Proc Natl Acad Sci U S A 103, 1382-7 (2006).

113. Schwartz, M. \& Vissing, J. Paternal inheritance of mitochondrial DNA. $N$ Engl J Med 347, 576-80 (2002). 
114. Filosto, M. et al. Lack of paternal inheritance of muscle mitochondrial DNA in sporadic mitochondrial myopathies. Ann Neurol 54, 524-6 (2003).

115. Taylor, R.W. et al. Genotypes from patients indicate no paternal mitochondrial DNA contribution. Ann Neurol 54, 521-4 (2003).

116. Thorburn, D.R. Mitochondrial disorders: prevalence, myths and advances. $J$ Inherit Metab Dis 27, 349-62 (2004).

117. Barritt, J.A., Brenner, C.A., Malter, H.E. \& Cohen, J. Mitochondria in human offspring derived from ooplasmic transplantation. Hum Reprod 16, 513-6. (2001).

118. St John, J.C. Ooplasm donation in humans: the need to investigate the transmission of mitochondrial DNA following cytoplasmic transfer. Hum Reprod 17, 1954-8 (2002).

119. Templeton, A. Ooplasmic transfer--proceed with care. N Engl J Med 346, 773-5 (2002).

120. Hsieh, R.H. et al. Decreased expression of mitochondrial genes in human unfertilized oocytes and arrested embryos. Fertil Steril 81 Suppl 1, 912-8 (2004).

121. Olivo, P.D., Van de Walle, M.J., Laipis, P.J. \& Hauswirth, W.W. Nucleotide sequence evidence for rapid genotypic shifts in the bovine mitochondrial DNA D-loop. Nature 306, 400-2 (1983).

122. Laipis, P.J., Van de Walle, M.J. \& Hauswirth, W.W. Unequal partitioning of bovine mitochondrial genotypes among siblings. Proc Natl Acad Sci U S A 85, 8107-10 (1988).

123. Yeom, Y.I. et al. Germline regulatory element of Oct-4 specific for the totipotent cycle of embryonal cells. Development 122, 881-94 (1996).

124. Hayashi, K., de Sousa Lopes, S.M. \& Surani, M.A. Germ cell specification in mice. Science 316, 394-6 (2007).

125. Kucej, M. \& Butow, R.A. Evolutionary tinkering with mitochondrial nucleoids. Trends Cell Biol 17, 586-92 (2007).

126. Kloc, M., Bilinski, S. \& Etkin, L.D. The Balbiani body and germ cell determinants: 150 years later. Curr Top Dev Biol 59, 1-36 (2004).

127. Pepling, M.E., Wilhelm, J.E., O'Hara, A.L., Gephardt, G.W. \& Spradling, A.C. Mouse oocytes within germ cell cysts and primordial follicles contain a Balbiani body. Proc Natl Acad Sci U S A 104, 187-92 (2007).

128. Cox, R.T. \& Spradling, A.C. A Balbiani body and the fusome mediate mitochondrial inheritance during Drosophila oogenesis. Development 130, 1579-90 (2003).

129. Fan, W. et al. A mouse model of mitochondrial disease reveals germline selection against severe mtDNA mutations. Science 319, 958-62 (2008).

130. Stewart, J.B., Freyer, C., Elson, J.L., Cansu, Z., Trifunovic, A., Larsson, NG. Strong purifying selection in transmission of mitochondrial DNA. PLOS Biology 6, 1-9 (2008).

131. Shoubridge, E.A. \& Wai, T. Medicine. Sidestepping mutational meltdown. Science 319, 914-5 (2008). 
132. Zhang, Y.H. et al. Mouth cell collection device for newborn mice. Mol Genet Metab 89, 164-7 (2006).

133. El Shourbagy, S.H., Spikings, E.C., Freitas, M. \& St John, J.C. Mitochondria directly influence fertilisation outcome in the pig. Reproduction 131, 233-45 (2006).

134. Ramakers, C., Ruijter, J.M., Deprez, R.H. \& Moorman, A.F. Assumptionfree analysis of quantitative real-time polymerase chain reaction (PCR) data. Neurosci Lett 339, 62-6 (2003).

135. Battersby, B.J., Redpath, M.E. \& Shoubridge, E.A. Mitochondrial DNA segregation in hematopoietic lineages does not depend on MHC presentation of mitochondrially encoded peptides. Hum Mol Genet 14, 2587-94 (2005).

136. McKee, M.D. \& Nanci, A. Postembedding colloidal-gold immunocytochemistry of noncollagenous extracellular matrix proteins in mineralized tissues. Microsc Res Tech 31, 44-62 (1995).

137. Brown, M.B., Forsythe, A.B. Robust tests for the equality of variances. $J$ Am Stat Assoc 69, 364-367 (1974).

138. Pan, G. Confidence intervals for comparing two scale parameters based on Levene's statistics. J Nonparam Stat 14, 459-476 (2007).

139. Lim, T.-S., Loh, W-Y. A comparison of tests of equality of variances. Comp Stat Data Analysis 22, 287-301 (1995).

140. Battersby, B.J., Loredo-Osti, J.C. \& Shoubridge, E.A. Nuclear genetic control of mitochondrial DNA segregation. Nat Genet 33, 183-6 (2003).

141. Wai, T., Teoli, D. \& Shoubridge, E.A. The mitochondrial DNA genetic bottleneck results from replication of a subpopulation of genomes. Nat Genet 40, 1484-8 (2008).

142. Solignac, M., Genermont, J., Monnerot, M. \& Mounolou, J.C. Drosophila Mitochondrial Genetics: Evolution of Heteroplasmy through Germ Line Cell Divisions. Genetics 117, 687-696 (1987).

143. Wonnapinij, P., Chinnery, P.F. \& Samuels, D.C. The distribution of mitochondrial DNA heteroplasmy due to random genetic drift. Am J Hum Genet 83, 582-93 (2008).

144. Jenuth, J.P., Peterson, A.C. \& Shoubridge, E.A. Tissue-specific selection for different mtDNA genotypes in heteroplasmic mice. Nat Genet 16, 93-5 (1997).

145. Shadel, G.S. Expression and maintenance of mitochondrial DNA: new insights into human disease pathology. Am J Pathol 172, 1445-56 (2008).

146. Larsson, N.G. et al. Mitochondrial transcription factor $A$ is necessary for mtDNA maintenance and embryogenesis in mice. Nat Genet 18, 231-6. (1998).

147. Lomeli, H., Ramos-Mejia, V., Gertsenstein, M., Lobe, C.G. \& Nagy, A. Targeted insertion of Cre recombinase into the TNAP gene: excision in primordial germ cells. Genesis 26, 116-7 (2000). 
148. Lewandoski, M., Wassarman, K.M. \& Martin, G.R. Zp3-cre, a transgenic mouse line for the activation or inactivation of loxP-flanked target genes specifically in the female germ line. Curr Biol 7, 148-51 (1997).

149. Epifano, O., Liang, L.F., Familari, M., Moos, M.C., Jr. \& Dean, J. Coordinate expression of the three zona pellucida genes during mouse oogenesis. Development 121, 1947-56 (1995).

150. Larsson, N.G. et al. Segregation and manifestations of the mtDNA tRNA(Lys) A-->G(8344) mutation of myoclonus epilepsy and ragged-red fibers (MERRF) syndrome. Am J Hum Genet 51, 1201-12 (1992).

151. Chinnery, P.F. \& Samuels, D.C. Relaxed replication of mtDNA: A model with implications for the expression of disease. Am J Hum Genet 64, 115865 (1999).

152. Newman, S.M., Zelenaya-Troitskaya, O., Perlman, P.S. \& Butow, R.A. Analysis of mitochondrial DNA nucleoids in wild-type and a mutant strain of Saccharomyces cerevisiae that lacks the mitochondrial HMG box protein Abf2p. Nucleic Acids Res 24, 386-93 (1996).

153. Ekstrand, M. \& Larsson, N.G. Breeding and genotyping of Tfam conditional knockout mice. Methods Mol Biol 197, 391-400 (2002).

154. St John, J.C., Bowles, E.J. \& Amaral, A. Sperm mitochondria and fertilisation. Soc Reprod Fertil Supp/ 65, 399-416 (2007).

155. Lansman, R.A., Avise, J.C. \& Huettel, M.D. Critical experimental test of the possibility of "paternal leakage" of mitochondrial DNA. Proc Natl Acad Sci U S A 80, 1969-71 (1983).

156. Zouros, E. The exceptional mitochondrial DNA system of the mussel family Mytilidae. Genes Genet Syst 75, 313-8 (2000).

157. Kvist, L., Martens, J., Nazarenko, A.A. \& Orell, M. Paternal leakage of mitochondrial DNA in the great tit (Parus major). Mol Biol Evol 20, 243-7 (2003).

158. Larsson, N.G., Oldfors, A., Garman, J.D., Barsh, G.S. \& Clayton, D.A. Down-regulation of mitochondrial transcription factor A during spermatogenesis in humans. Hum Mol Genet 6, 185-91. (1997).

159. Rantanen, A., Jansson, M., Oldfors, A. \& Larsson, N.G. Downregulation of Tfam and mtDNA copy number during mammalian spermatogenesis. Mamm Genome 12, 787-92 (2001).

160. Amaral, A., Ramalho-Santos, J. \& St John, J.C. The expression of polymerase gamma and mitochondrial transcription factor $A$ and the regulation of mitochondrial DNA content in mature human sperm. Hum Reprod 22, 1585-96 (2007).

161. el Meziane, A., Callen, J.C. \& Mounolou, J.C. Mitochondrial gene expression during Xenopus laevis development: a molecular study. EMBO J 8, 1649-55 (1989).

162. Kameyama, Y., Filion, F., Yoo, J.G. \& Smith, L.C. Characterization of mitochondrial replication and transcription control during rat early development in vivo and in vitro. Reproduction 133, 423-32 (2007). 
163. Vallejo, C.G., Lopez, M., Ochoa, P., Manzanares, M. \& Garesse, R. Mitochondrial differentiation during the early development of the brine shrimp Artemia franciscana. Biochem J 314 ( Pt 2), 505-10 (1996).

164. Houghton, F.D. Energy metabolism of the inner cell mass and trophectoderm of the mouse blastocyst. Differentiation 74, 11-8 (2006).

165. Cerritelli, S.M. et al. Failure to produce mitochondrial DNA results in embryonic lethality in Rnaseh1 null mice. Mol Cell 11, 807-15 (2003).

166. Hance, N., Ekstrand, M.I. \& Trifunovic, A. Mitochondrial DNA polymerase gamma is essential for mammalian embryogenesis. Hum Mol Genet 14, 1775-83 (2005).

167. Nasir, J. et al. Targeted disruption of the Huntington's disease gene results in embryonic lethality and behavioral and morphological changes in heterozygotes. Cell 81, 811-23 (1995).

168. Agostino, A. et al. Constitutive knockout of Surf1 is associated with high embryonic lethality, mitochondrial disease and cytochrome c oxidase deficiency in mice. Hum Mol Genet 12, 399-413 (2003).

169. Cossee, M. et al. Inactivation of the Friedreich ataxia mouse gene leads to early embryonic lethality without iron accumulation. Hum Mol Genet 9, 1219-26 (2000).

170. Di Pietro, E., Sirois, J., Tremblay, M.L. \& MacKenzie, R.E. Mitochondrial NAD-dependent methylenetetrahydrofolate dehydrogenasemethenyltetrahydrofolate cyclohydrolase is essential for embryonic development. Mol Cell Biol 22, 4158-66 (2002).

171. Abu-Elheiga, L. et al. Mutant mice lacking acetyl-CoA carboxylase 1 are embryonically lethal. Proc Natl Acad Sci U S A 102, 12011-6 (2005).

172. Takahashi, Y. et al. Mammalian copper chaperone Cox17p has an essential role in activation of cytochrome $\mathrm{C}$ oxidase and embryonic development. Mol Cell Biol 22, 7614-21 (2002).

173. Ji, S. et al. Homozygous carnitine palmitoyltransferase $1 \mathrm{~b}$ (muscle isoform) deficiency is lethal in the mouse. Mol Genet Metab 93, 314-22 (2008).

174. Santos, T.A., El Shourbagy, S. \& St John, J.C. Mitochondrial content reflects oocyte variability and fertilization outcome. Fertil Steril 85, 584-91 (2006).

175. O-165 quantification of mtDNA copy number in the human secondary oocyte. Hum Reprod 14, 2419 (1999).

176. Pagnamenta, A.T. et al. Dominant inheritance of premature ovarian failure associated with mutant mitochondrial DNA polymerase gamma. Hum Reprod 21, 2467-73 (2006).

177. Luoma, P. et al. Parkinsonism, premature menopause, and mitochondrial DNA polymerase gamma mutations: clinical and molecular genetic study. Lancet 364, 875-82 (2004).

178. Van Goethem, G., Dermaut, B., Lofgren, A., Martin, J.J. \& Van Broeckhoven, C. Mutation of POLG is associated with progressive external ophthalmoplegia characterized by mtDNA deletions. Nat Genet 28, 211-2. (2001). 
179. Trifunovic, A. et al. Premature ageing in mice expressing defective mitochondrial DNA polymerase. Nature 429, 417-23 (2004).

180. Rovio, A.T. et al. Mutations at the mitochondrial DNA polymerase (POLG) locus associated with male infertility. Nat Genet 29, 261-2 (2001).

181. Dean, N.L. et al. Prospect of preimplantation genetic diagnosis for heritable mitochondrial DNA diseases. Mol Hum Reprod 9, 631-8 (2003).

182. Mohamed, O.A. et al. Uterine Wnt/beta-catenin signaling is required for implantation. Proc Natl Acad Sci U S A 102, 8579-84 (2005).

183. Hermo, L. et al. Alterations in the testis of hormone sensitive lipasedeficient mice is associated with decreased sperm counts, sperm motility, and fertility. Mol Reprod Dev 75, 565-77 (2008).

184. Spikings, E.C., Alderson, J. \& St John, J.C. Transmission of mitochondrial DNA following assisted reproduction and nuclear transfer. Hum Reprod Update 12, 401-15 (2006).

185. Lee, S.T., Oh, S.J., Lee, E.J., Han, H.J. \& Lim, J.M. Adenosine triphosphate synthesis, mitochondrial number and activity, and pyruvate uptake in oocytes after gonadotropin injections. Fertil Steril 86, 1164-9 (2006).

186. Zeng, H.T. et al. In vitro-matured rat oocytes have low mitochondrial deoxyribonucleic acid and adenosine triphosphate contents and have abnormal mitochondrial redistribution. Fertil Steril (2008).

187. Spinazzola, A. et al. MPV17 encodes an inner mitochondrial membrane protein and is mutated in infantile hepatic mitochondrial DNA depletion. Nat Genet 38, 570-5 (2006).

188. Pereira, L. et al. No evidence for an mtDNA role in sperm motility: data from complete sequencing of asthenozoospermic males. Mol Biol Evol 24, 868-74 (2007).

189. Ruiz-Pesini, E., Diez-Sanchez, C., Lopez-Perez, M.J. \& Enriquez, J.A. The role of the mitochondrion in sperm function: is there a place for oxidative phosphorylation or is this a purely glycolytic process? Curr Top Dev Biol 77, 3-19 (2007).

190. Nakada, K. et al. Mitochondria-related male infertility. Proc Natl Acad Sci U $S$ A 103, 15148-53 (2006).

191. Song, G.J. \& Lewis, V. Mitochondrial DNA integrity and copy number in sperm from infertile men. Fertil Steril 90, 2238-44 (2008).

192. Clark, K.M. et al. An mtDNA mutation in the initiation codon of the cytochrome $\mathrm{C}$ oxidase subunit II gene results in lower levels of the protein and a mitochondrial encephalomyopathy. Am J Hum Genet 64, 1330-9. (1999).

193. Antonicka, $\mathrm{H}$. et al. Mutations in COX10 result in a defect in mitochondrial heme A biosynthesis and account for multiple, early-onset clinical phenotypes associated with isolated COX deficiency. Hum Mol Genet 12, 2693-702 (2003). 
194. Valnot, I. et al. A mutation in the human heme A:farnesyltransferase gene (COX10) causes cytochrome c oxidase deficiency. Hum Mol Genet 9, 1245-9. (2000).

195. Antonicka, $\mathrm{H}$. et al. Mutations in COX15 produce a defect in the mitochondrial heme biosynthetic pathway, causing early-onset fatal hypertrophic cardiomyopathy. Am J Hum Genet 72, 101-14 (2003).

196. Sacconi, S. et al. Mutation screening in patients with isolated cytochrome c oxidase deficiency. Pediatr Res 53, 224-30 (2003).

197. Hudson, G. \& Chinnery, P.F. Mitochondrial DNA polymerase-gamma and human disease. Hum Mol Genet 15 Spec No 2, R244-52 (2006).

198. Parsons, T.J. et al. A high observed substitution rate in the human mitochondrial DNA control region. Nat Genet 15, 363-8 (1997).

199. Takamatsu, C. et al. Regulation of mitochondrial D-loops by transcription factor A and single-stranded DNA-binding protein. EMBO Rep 3, 451-6 (2002).

200. Fisher, R.P., Lisowsky, T., Breen, G.A. \& Clayton, D.A. A rapid, efficient method for purifying DNA-binding proteins. Denaturation-renaturation chromatography of human and yeast mitochondrial extracts. $J$ Biol Chem 266, 9153-60 (1991).

201. Shen, E.L. \& Bogenhagen, D.F. Developmentally-regulated packaging of mitochondrial DNA by the HMG-box protein mtTFA during Xenopus oogenesis. Nucleic Acids Res 29, 2822-8 (2001).

202. Maniura-Weber, K., Goffart, S., Garstka, H.L., Montoya, J. \& Wiesner, R.J. Transient overexpression of mitochondrial transcription factor A (TFAM) is sufficient to stimulate mitochondrial DNA transcription, but not sufficient to increase mtDNA copy number in cultured cells. Nucleic Acids Res 32 , 6015-27 (2004).

203. Ashley, N., Harris, D. \& Poulton, J. Detection of mitochondrial DNA depletion in living human cells using PicoGreen staining. Exp Cell Res 303, 432-46 (2005).

204. Rust, M.J., Bates, M. \& Zhuang, X. Sub-diffraction-limit imaging by stochastic optical reconstruction microscopy (STORM). Nat Methods 3 , 793-5 (2006).

205. Bates, M., Huang, B., Dempsey, G.T. \& Zhuang, X. Multicolor superresolution imaging with photo-switchable fluorescent probes. Science 317, 1749-53 (2007).

206. Leary, S.C. et al. The human cytochrome c oxidase assembly factors SCO1 and SCO2 have regulatory roles in the maintenance of cellular copper homeostasis. Cell Metab 5, 9-20 (2007).

207. Chan, D.C. Mitochondrial dynamics in disease. N Engl J Med 356, 1707-9 (2007).

208. Cox, R.T. \& Spradling, A.C. Milton controls the early acquisition of mitochondria by Drosophila oocytes. Development 133, 3371-7 (2006).

209. Haas, R.H. et al. Mitochondrial disease: a practical approach for primary care physicians. Pediatrics 120, 1326-33 (2007). 
210. Elliott, H.R., Samuels, D.C., Eden, J.A., Relton, C.L. \& Chinnery, P.F. Pathogenic mitochondrial DNA mutations are common in the general population. Am J Hum Genet 83, 254-60 (2008).

211. Taylor, R.W. \& Turnbull, D.M. Mitochondrial DNA mutations in human disease. Nat Rev Genet 6, 389-402 (2005).

212. Scherz-Shouval, R. \& Elazar, Z. ROS, mitochondria and the regulation of autophagy. Trends Cell Biol 17, $422-7$ (2007).

213. Sandoval, $\mathrm{H}$. et al. Essential role for Nix in autophagic maturation of erythroid cells. Nature 454, 232-5 (2008). 
Canadian Nuclear Commission canadienne Safety Commission

\section{de sûreté nucléaire}

$01187-2-08.0$

Licence Number Numéro de permis

\section{I) LICENSEE}

PERMIS PORTANT SUR LES SUBSTANCES NUCLÉAIRES ET LES APPAREILS A RAYONNEMENT Pursuant to section $24(2)$ of the Nuclear Safety and Control Act,
this licence is issued to:

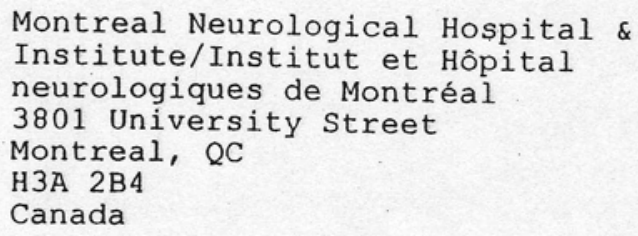

hereinafter «the licensee».

This licence is valid from: May 12003 to April 302008.

III) LICENSED ACTIVITIES

This licence authorizes the licensee to possess, transfer, import, export, use and store the nuclear substances and the prescribed equipment listed in section IV) of this licence.

This licence is issued for: consolidated licence: between 11 and 50 locations, inclusive, where radolsotopes are usea (849)

IV) NUCLEAR SUBSTANCES AND PRESCRIBED EQUIPMENT

\begin{tabular}{|c|c|c|c|c|}
\hline$T E M$ & $\begin{array}{c}\text { NUCLEAR } \\
\text { SUBSTANCE }\end{array}$ & Un & $\begin{array}{l}\text { D. SOL } \\
\text { IMUM } \\
\text { ITITY }\end{array}$ & URCE \\
\hline 1 & Carbon 14 & & 11 & GBq \\
\hline 2 & Calcium 45 & & 800 & $\mathrm{MBq}$ \\
\hline 3 & Chromium 51 & & 2 & GBq \\
\hline 4 & Fluorine 18 & & 2 & GBq \\
\hline 5 & Iron 55 & & 100 & $\mathrm{MBq}$ \\
\hline 6 & Gallium 68 & & 400 & $\mathrm{MBq}$ \\
\hline 7 & Hydrogen 3 & & 33 & GBq \\
\hline 8 & Iodine 125 & & 4 & GBq \\
\hline 9 & Iodine 131 & & 2 & GBq \\
\hline 10 & Krypton 85 & & 74 & GBq \\
\hline 11 & Phosphorus & 32 & 11 & $\mathrm{GBq}$ \\
\hline 12 & Phosphorus & 33 & 2 & $\mathrm{GBq}$ \\
\hline 13 & Sulfur 35 & & 22 & GBq \\
\hline 14 & Technetium & $99 m$ & 11 & GBq \\
\hline 15 & Cesium 137 & & $\mathrm{n} / \mathrm{a}$ & \\
\hline 16 & Radium 226 & & $\mathrm{n} / \mathrm{a}$ & \\
\hline 17 & Europium 15 & & $\mathrm{n} / \mathrm{a}$ & \\
\hline 18 & $\begin{array}{l}\text { Germanium } 6 \\
\text { Gallium } 68\end{array}$ & $58 /$ & $\mathrm{n} / \mathrm{a}$ & \\
\hline 19 & Cesium 137 & & $\mathrm{n} / \mathrm{a}$ & \\
\hline
\end{tabular}

SEALED SOURCE
MAXIMUM
QUANTITY
$\mathrm{n} / \mathrm{a}$
$\mathrm{n} / \mathrm{a}$
$\mathrm{n} / \mathrm{a}$
$\mathrm{n} / \mathrm{a}$
$\mathrm{n} / \mathrm{a}$
$\mathrm{n} / \mathrm{a}$
$\mathrm{n} / \mathrm{a}$
$\mathrm{n} / \mathrm{a}$
$\mathrm{n} / \mathrm{a}$
$\mathrm{n} / \mathrm{a}$
$\mathrm{n} / \mathrm{a}$
$\mathrm{n} / \mathrm{a}$
$\mathrm{n} / \mathrm{a}$
$\mathrm{n} / \mathrm{a}$
$140 \mathrm{kBq}$
$370 \mathrm{kBq}$
$740 \mathrm{kBq}$
$1 \mathrm{GBq}$
$1 \mathrm{GBq}$

\section{EOUIPMENT \\ MAKE AND MODEL}

$n / a$
$n / a$
$n / a$
$n / a$
$n / a$
$n / a$
$n / a$
$n / a$
$n / a$
$n / a$
$n / a$
$n / a$
$n / a$
$n / a$

Beckman LS series Wallac 1200 Series Wallac 1400 series $\mathrm{n} / \mathrm{a}$

$\mathrm{n} / \mathrm{a}$

The total quantity of an unsealed nuclear substance in possession shall not exceed the corresponding listed unsealed source maximum quantity. The total quantity of nuclear substance per sealed source shall not exceed its corresponding listed sealed source maximum quantity. Sealed sources shall only be used in the corresponding listed equipment. 


\section{McGill University Animal Use Protocol - Research}

For Office Use Only:

Protocol \#:

Approval End Date:

Tacility Committee:
TNAtle:
Molecular determinants of mitochondrial DNA organization and segregation aka Nuclear genetic control of mitochondrial DNA segregation (must match the title of the funding source application)
New Application
凶Renewal of Protocol \# $\underline{5092}$
Pilot
Category (see section 11):

\section{Investigator Data:}

Principal Investigator:

Unit/Department:

Eric A. Shoubridge

Neurology \& Neurosurgrey

Address:

M.N.I 3801 University Street, Montreal, Quebec H3A 2B4
Phone \#: 514-398-1997

Fax\#: 514-398-1509

Email: eric@ericpc.mni.mcgill.ca

\section{Emergency Contacts: Two people must be designated to handle emergencies.}

Name: Eric Shoubridge

Work \#: 398-1997 Emergency \#:

(514) $935-1836$

Name: Timothy Wai

Work \#: 398-8523 Emergency \#:

(514) 591-2819



Investigator's Statement: The information in this application is exact and complete. I assure that all care and use of animals in this proposal will be in accordance with the guidelines and policies of the Canadian Council on Animal Care and those of McGill University. I shal for one year and must be approved on an annual basis. I will ensure-that ay cohaporacors as approved. I understand that this approval is vali

Principal Investigator's signature:



\begin{tabular}{|l|l|l|}
\hline Chair, Facility Animal Care Committee: & & Date: \\
\hline Animal Compliance Office: & & Date: \\
\hline Chair, Ethics Subcommittee (as per UACC policy): & & Date: \\
\hline Approved Animal Use & Beginning: & Ending: \\
\hline
\end{tabular}

This protocol has been approved with the modifications noted in Section 13 . 


\section{Research Personnel and Qualifications}

List the names of the Principal Investigator and of all individuals who will be in contact with animals in this study and their employment classification (investigator, technician, research assistant, undergraduate/ graduate student, fellow). and the supervision received must be described. Training if an undergraduate student is involved, the role of the student www.animalcare.mcgill.ca for details. Each person listed in this atory for all personnel listed here. Refer to protocol. (Space will expand as needed)

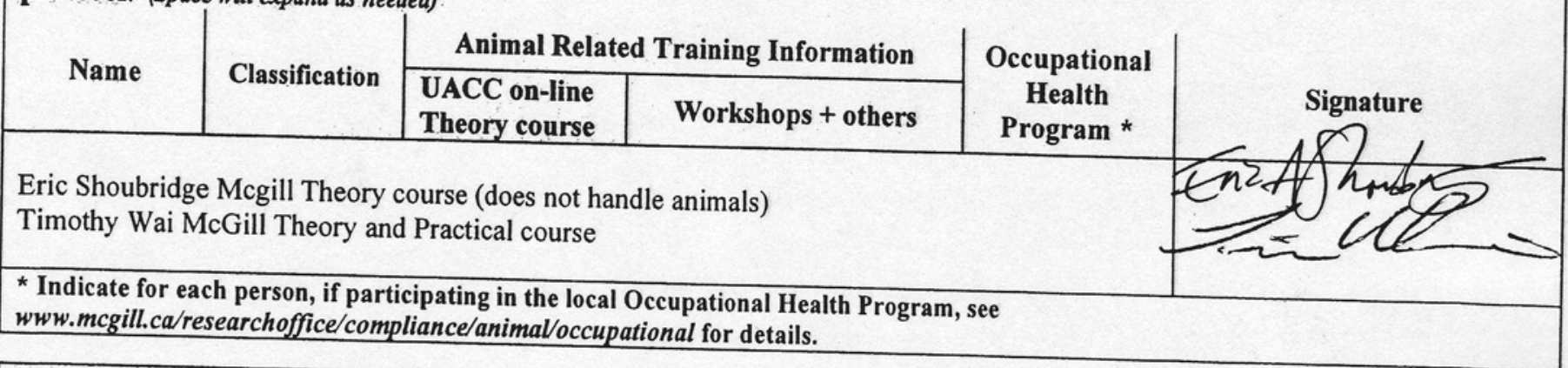

5. Summary (in language that will be understood by members of the general public) 5 a) AIMS AND BENEFITS: Describe, in a short paragraph, the overall aim of the study and its potential benefit to
human/animal health or to the advancement of scientific knowledge.

The goal of this study is to understand how mitochondrial DNAs are transmitted from mother to offspring using the mouse as an these diseases remaining largely unpredictable. An underst cause incurable multisystem diseases with genetic counselling of bottleneck will directly impact the understanding of how humans inherit mitochondrial of the so-called mitochondrial genetic

5 b) SPECIFIC OBJECTIVES OF THE STUDY: Summarize in point form the primary objectives of this study. Using mutliple transgenic mouse strains we will determine the size and shape of the mitochondrial genetic bottleneck during the development of the female germline which is responsible for the propagation and elimination of mutant mitochondrial DNA Conditional knockouts affecting either mitochondrial DNA amounts and function will be carried out in cells of the female germline both at early and late stages in order to determine the importance of these parameters on the size and shape of the
bottleneck

5 c) Indicate if and how the current goals differ from those in last year's application.

no different

5 d) List the section / subsection numbers where significant changes have been made

5 e) KEYWORDS: Using keywords only, list the procedures used on animals (e.g. anaesthesia, breeding colony, For a more complete list of suggested keywords survival surgery, euthanasia by exsanguination, behavioural studies). (www.mcgill.ca/researchoffice/compliance/anim refer to Appendix 1 of the Guidelines

Breeding, DNA isolation, tail clipping, C-section, Embryo islolation 
6 a) Purpose of Animal Use (Check most appropriate one):

1. $\square$ Studies of a fundamental nature/basic research

2. $\$ Studies for medical purposes relating to human/animal diseases/disorders

3. $\square$ Regulatory testing

4. $\square$ Development of products/appliances for human/veterinary medicine

5. If for Teaching, use the Animal Use Protocol form for Teaching (www.mcgill.ca/researchoffice/compliance/animal/forms)

6 b) Will field studies be conducted? NO $₫$ YES $\square$ If yes, complete "Field Study Form"

Will the project involve genetically altering animals? NO $\$ YES $\square$ If yes, complete SOP \#5 or \#6 Will the project involve breeding animals? NO $\square$ YES $\triangle$ If breeding transgenics or knockouts, complete SOP\#4

\section{Animal Data}

7 a) Please justify the need for live animals versus alternate methods (e.g. tissue culture, computer simulation) We are investigating the intergenerational transmission of mitochondrial DNA, which can only be done in live animals.

$7 \mathrm{~b})$ Describe the characteristics of the animal species selected that justifies its use in the proposed study ( consider characteristics such as body size, species, strain, data from previous studies or unique anatomic/physiological features)

The floxed transgenic mice (TFAMloxP and COX10-loxP) are required to provide an excisable targets for CreRecombinase, exogenously expressed from different promoters in the TNAP-Cre mice. The Oct4-GFP transgene provides a marker used in the isolation of the cells of the female germline.

7 c) Description of animals

Quality Control Assurance: To prevent introduction of infectious diseases into animal facilities, a health status report or veterinary inspection certificate may be required prior to receiving animals from all non-commercial sources or from commercial sources whose animal health status is unknown or questionable.
Quarantine and further testing may be required for these animals. If more than 6 columns are needed, please attach another page

\begin{tabular}{|l|l|l|l|l|l|l|} 
& Sp/strain 1 & Sp/strain 2 & Sp/strain 3 & Sp/strain 4 & Sp/strain 5 & Sp/strain 6 \\
\hline Species & mouse & mouse & mouse & mouse & & \\
\hline Supplier/Source & In house & in house & in house & in house & & \\
\hline Strain & $\begin{array}{l}\text { Balb/C X } \\
\text { C57BI6/J } \\
\text { T/FAM-loxP }\end{array}$ & $\begin{array}{l}\text { CD1 COX10- } \\
\text { loxP }\end{array}$ & $\begin{array}{l}\text { C57Bl6/J } \\
\text { Oct4-GFP }\end{array}$ & $\begin{array}{l}\text { C57B16/J } \\
\text { TNAP-Cre }\end{array}$ & & \\
\hline Sex & M/F & M/F & M/F & M/F & & \\
\hline Age/Wt & various & various & various & various & & \\
\hline \# To be purchased & & & & & & \\
\hline $\begin{array}{l}\text { \# Produced by in- } \\
\text { house breeding }\end{array}$ & 200 & 20 & 20 & 20 & & \\
\hline $\begin{array}{l}\text { \# Other } \\
\text { (e.g.field studies) }\end{array}$ & & & & 20 & 20 & \\
\hline $\begin{array}{l}\text { \#needed at one } \\
\text { time }\end{array}$ & 50 & 20 & & $20 / y r$ & & \\
\hline \# per cage & & & $20 / y r$ & $20 / y r$ & & \\
\hline TOTAL\# /YEAR & $100 / y r$ & & & & & \\
\hline
\end{tabular}

7 d) Explanation of Animal Usage: BASED ON THE EXPERIMENTAL OBJECTIVES OF THE PROJECT, describe the number of animals required for one year. Include information on experimental and control groups, \# per
group, and failure rates.

For breeding, specify how many adults are used, number of offspring produced, and how many offspring are used in experimental procedures.

The arithmetic explaining how the total of animals for each column in the table above is calculated should be made clear.
(Space will expand as needed) 
We are examining the female germline of our heteroplasmic mouse model both in the presence and absence of excisable transgenes that, when conditionally excised, may affect the segregation of mitochondrial DNA. To study the wild type biology of the female germline, we must intercross Oct4-GFP males with heteroplasmic females and examine at least 150 embryos, or 15 to 30 crosses, depending upon fecundity, in order define the size and shape of the mtDNA genetic bottleneck. We will need to generate 4 separate multiple transgenic lines to study the effect of germline conditional knockouts and their effect on the size and shape of this genetic bottleneck. Approximately 150 embryos per line will be examined from 15 to 30 crosses. Cage requirements for mating will be dependent upon fecundity of females.

\section{Animal Husbandry and Care}

8 a) If projects involves non-standard cages, diet and/or handling, please specify

$8 \mathrm{~b})$ Is there any component to the proposed procedures which will result in immunosuppression or decreased immune function (e.g. stress, radiation, steroids, chemotherapeutics, genetic modification of the immune system)? NO $\square \quad$ YES $\square$ if yes, specify:

8 c) Indicate area(s) where animal use procedures will be conducted:

Building: MNI Room: animal quarters

Indicate area(s) all facilities where animals will be housed:

Building: MNI Room: animal quarters

If animal housing and animal use are in different locations, briefly describe procedures for transporting animals:

\section{Standard Operating Procedures (SOPs)}

Complete this section if you plan to use any of the UACC SOPs listed below. IT IS UACC POLICY THAT THESE SOPS BE USED WHEN APPLICABLE. Any proposed variation of the SOPs must be described and justified. The Standard Operating Procedures can be found at the UACC website at www.mcgill.ca/researchoffice/compliance/animal/procedures
The completed and signed SOP form must be attached to the protocol.

Check all SOPs that will be used:

Blood Collection UACC\#1

\begin{tabular}{|ll|l|}
\hline Blood Collection UACC\#1 & $\square$ & Collection of Amphibian Oocytes UACC\#9 \\
\hline Anaesthesia in rodents UACC\#2 & $\square$ & Rodent Survival Surgery UACC\#10 \\
\hline Analgesia in rodents UACC\#3 & $\square$ & Anaesthesia \& Analgesia Neonatal Rodents UACC\#11 \\
\hline Breeding transgenics/knockouts UACC\#4 & $\square$ & Stereotaxic Survival Surgery in Rodents UACC\#12 \\
\hline Transgenic Generation UACC\#5 & $\square$ & Field Studies Form \\
\hline Knockout/in Generation UACC\#6 & $\square$ & Phenotype Disclosure Form \\
\hline Production of Monoclonal Antibodies UACC\#7 & $\square$ & Other, specify: \\
\hline Production of Polyclonal Antibodies UACC\#8 & $\square$ & \\
\hline
\end{tabular}

10. Description of Procedures 10 a). IF A PROCEDURE IS COVERED BY AN SOP, WRITE “AS PER SOP”, NO FURTHER DETAIL IS
REQUIRED.

FOR EACH EXPERIMENTAL GROUP, DESCRIBE ALL PROCEDURES AND TECHNIQUES, WHICH ARE NOT PART OF THE SOPS, IN THE ORDER IN WHICH THEY WILL BE PERFORMED - surgical procedures, immunizations, behavioural tests, immobilization and restraint, food/water deprivation, requirements for post-operative care, sample collection, substance administration, special monitoring, etc Appendix 2 of the Guidelines (www.mcgill.ca/researchoffice/compliance/animal/guidelines) provides a sample list of points that should be addressed in this section. 
Categories of Invasiveness (from the CCAC Categories of Invasiveness in Animal Experiments). Please refer to this document for a
more detailed description of categories.

Category A: Studies or experiments on most invertebrates or no entire living material.

Category B: Studies or experiments causing little or no discomfort or stress. These might include holding animals captive, injection, percutaneous blood sampling, accepted euthanasia for tissue harvest, acute non-survival experiments in which the animals are completely
anaesthetized.

Category C: Studies or experiments involving minor stress or pain of short duration. These might include cannulation or restraint, overnight food and/s water deprivation which exceedia, minor surgery under anaesthesia, such as biopsy; short periods of animals that involve short-term stressful restraint. anaesthesia with subsequent recovery prot involve moderate to severe distress or discomfort. These might include major surgery under immunization with complete Freund's adjuvant, application of or more) periods of physical restraint; induction of behavioural stresses, accordance with University policy). Category E: Procedures that involve inflicting severe pain, near, at or above the pain threshold of unanaesthetized, conscious animals. Not confined to but may include exposure to noxious stimuli or agents whose effects are unknown; exposure to drugs or chemicals at levels that (may) markedly impair physiological systems and which cause death, severe pain or extreme distress or physical trauma on unanaesthetized animals. According to University policy, E level studies are not permitted.

12. Potential Hazards to Personnel and Animals It is the responsibility of the investigator to obtain the necessary Biohazard and/or Radiation Safety permits before this protocol is submitted for review.

\section{A copy of these certificates must be attached, if applicable.}

No hazardous materials will be used in this study: $\square$

12 a) Indicate which of the following will be used in animals:

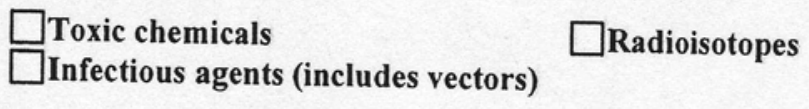

12 b) Complete the following table for each agent to be used (use additional page as required):

\begin{tabular}{|l|l|l|l|}
\hline Agent name & & & \\
\hline Dosage & & & \\
\hline Route of administration & & & \\
\hline Frequency of administration & & & \\
\hline Duration of administration & & & \\
\hline Number of animals involved & & & \\
\hline Survival time after administration & & & \\
\hline
\end{tabular}

12 c) After administration the animals will be housed in:

the animal care facility $\square$ laboratory under supervision of laboratory personnel

Please note that cages must be appropriately labeled at all times.

12 d) Describe potential health risk (s) to humans or animals:

12 e) Describe measures that will be used to reduce risk to the environment and all project and animal facility personnel:

12 f) If using cell lines, have they been tested?

$\square$ Yes If yes, What human and/or animal pathogens have been tested?

No If no, justify:

13. Reviewer's Comments and Modifications (to be completed by ACC only): The Animal Care Committee has made the following modification(s) to this animal use procedure protocol during the review process. Please make these changes to your copy and comply with the recommended changes as a condition of approval. 


\section{MCGILL UNIVERSITY \\ UNIVERSITY ANIMAL CARE COMMITTEE}

UACC Standard Operating Procedure \# 4

October 2005 version

\section{TRANSGENIC OR KNOCKOUT I IN MOUSE BREEDING}

1. INTRODUCTION

Standard Operating Procedures (SOPs) provide a detailed description of commonly used procedures. SOPS offer investigators an alternative to writing detailed procedures on their protocol forms. Any deviation from the approved procedures must be clearly described and justified in the Animal Use Protocol form (AUP). Approval of the protocol only) must be attached to the AUP form. The SOP for that project only. A signed SOP form (pages up to signature

2. INFORMATION REQUIRED 2.1 Species/strain(s) (must refer to the Sp/strain column \# of the table in "Description of animals"
section in main protocol):

\section{$2.2 \quad$ Transgenic? $\bigotimes$}

Gene inserted (include promoter \& gene): Oct4-GFP

TNAP-Cre

Target tissue: germline

Ancillary tissue:
Knockout? \

Gene deleted: TFAM loxP COX10 loxP

Target tissue: germline

Ancillary tissue:
Knock/in?

Gene deleted initially:

Gene inserted:

Target tissue:

Ancillary tissue: 2.3 Supplier: Please include a complete address, contact person, phone, fax and email information. Note
that a Certificate of health must be available to FACC personnel PRIOR to animal arrival.

1. Commercial:

2. Academic: animals have been here for 3 years

2.4 Phenotype: Include any trait included in a published article or reported to you by the originating a decreased lifespan.

1. Heterozygotes: no phenotype

2. Homozygotes: TFAM and COX10 deletions are embryonic lethal

2.5 Clinical endpoint - General clinical endpoint criteria signs apply.

Supply criteria specific to phenotype: none

Include plans to monitor or alleviate this distress:

Frequency of monitoring: daily

2.6 Are there changes to this SOP indicated in the AUP form? $\square$ YES $\triangle N O$ If yes, specify changes:

2.7 PI Signature:

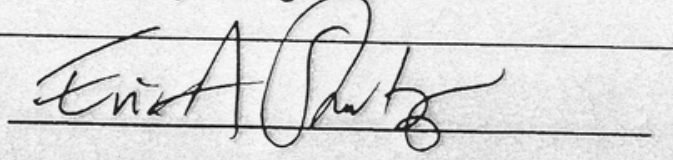
Date: 22 fotenfer, 2008 


\section{APPLICATION TO USE BIOHAZARDOUS MATERIALS ${ }^{*}$}



Projects involving potentially biohazardous materials should not be commenced without approval from Environmental Health \& Safety. Submit applications before 1) starting new projects, 2) renewing existing projects, or 3) changing the nature
of the biohazardous materials within existing projects.

\section{PRINCIPAL INVESTIGATOR: Dr. Eric A. Shoubridge \\ DEPARTMENT: Neurology/Neurosurgery}

PHONE: $398-1997$

FAX: 398-1509

3801, University street, Room: 660

ADDRESS: $\quad$ H3A 2B4

PROJECT TITLE:

E-MAIL: eric@ericpc.mni.mcgill.ca

*Transmission genetics of mammalian mitochondrial DNA. New Fund-NIH

*Assembly of cytochrome c oxidase in mitochondrial encephalomyopathy-MDA \#228772

2. EMERGENCY: Person(s) designated to handle emergencies

Name: Eric A. Shoubridge

Name: Tim Johns

Phone No: work: 398-1997

home: $\quad 935-1836$

Phone No: work: $398-8523$ home: 488-9771

\section{FUNDING SOURCE OR AGENCY (specify):}

228772

Grant No.: New Fund

Beginning date:

\begin{tabular}{l} 
MDA, NIH \\
\hline July 1, 2005 \\
July 1, 2005
\end{tabular}

July 1,2005
July 1,2005
June 30,2009

June 30, 2009

4. Indicate if this is

$\bigotimes$ Renewal: procedures previously approved without alterations.

Approval End Date: June 30, 2005

$\bigotimes$ New funding source: project previously reviewed and approved under an application to another agency.
Agency:
N/H

Approval End Date:

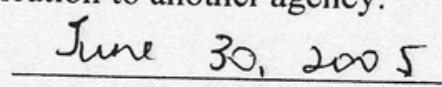

$\square$ New project: project not previously reviewed.

$\square$ Approved project: change in biohazardous materials or procedures.

$\square$ Work/project involving biohazardous materials in teaching/diagnostics.

CERTIFICATION STATEMENT: Environmental Health \& Safety approves the experimental procedures proposed and certifies with the applicant that the experiment will be in accordance with the principles outlined in Health Canada's "Laboratory Biosafety Guidelines" and in the "McGill Laboratory Biosafety Manual".

Containment Level (select one):

Principal Investigator or course director:

Approved by Environmental Health \& Safety:
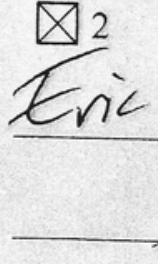



$$
\text { Exping: }
$$

3

date:



date:



06

o9 


\section{McGill University}

\section{APPLICATION TO USE BIOHAZARDOUS MATERIALS*}



Projects involving potentially biohazardous materials should not be commenced without approval from Environmental Health \& Safety. Submit applications before 1) starting new projects, 2) renewing existing projects, or 3) changing the nature
of the biohazardous materials within existing projects.

\section{PRINCIPAL INVESTIGATOR: Dr. Eric A. Shoubridge \\ DEPARTMENT: Neurology/Neurosurgery}

PHONE: $398-1997$

FAX: 398-1509

\section{1, University street, Room: 660}

ADDRESS: Montreal, Quebec

PROJECT TITLE: H3A 2B4

*Transmission genetics of mammalian mitochondrial DNA. New Fund-NIH

*Assembly of cytochrome $c$ oxidase in mitochondrial encephalomyopathy-MDA \#228772

E-MAIL: eric@ericpc.mni.mcgill.ca

2. EMERGENCY: Person(s) designated to handle emergencies

Name: Eric A. Shoubridge

Name: Tim Johns

Phone No: work: 398-1997

Phone No: work: $398-8523$

home: $\quad 935-1836$

home: 488-9771

\section{FUNDING SOURCE OR AGENCY (specify):}

228772

Grant No.: New Fund

Beginning date:

MDA, NIH

July 1,2005

July 1, 2005
June 30, 2009

June 30, 2009

\section{Indicate if this is}

$\bigotimes$ Renewal: procedures previously approved without alterations.

Approval End Date: June 30, 2005

$\bigotimes$ New funding source: project previously reviewed and approved under an application to another agency.

$$
\text { Agency: } \mathrm{N} / \mathrm{H}
$$

New project: project not previously reviewed

Approval End Date:

End date:

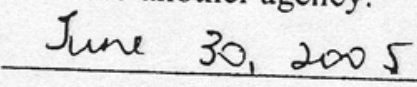

Approved project: change in biohazardous materials or procedures.

Work/project involving biohazardous materials in teaching/diagnostics.

CERTIFICATION STATEMENT: Environmental Health \& Safety approves the experimental procedures proposed and certifies with the applicant that the experiment will be in accordance with the principles outlined in Health Canada's "Laboratory Biosafety Guidelines" and in the "McGill Laboratory Biosafety Manual".

Containment Level (select one):

Principal Investigator or course director:

Approved by Environmental Health \& Safety:


Exmini:<smiles>C1CCCC1</smiles>
date:

date:
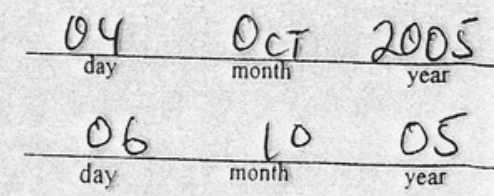


\section{APPLICATION TO USE BIOHAZARDOUS MATERIALS*}

Projects involving potentially biohazardous materials should not be commenced without approval from Environmental Health \& Safety. Submit applications before 1) starting new projects, 2) renewing existing projects, or 3) changing the nature of the biohazardous materials within existing projects.

\section{PRINCIPAL INVESTIGATOR: Dr. Eric A. Shoubridge}

DEPARTMENT: Neurology/Neurosurgery
PHONE: 398-1997

FAX: $398-1509$

3801, University street, Room: 660

ADDRESS: H3A 2B4

\section{Montreal, Quebec}

PROJECT TITLE:

E-MAIL: eric@ericpc.mni.mcgill.ca

*Transmission genetics of mammalian mitochondrial DNA. New Fund-NIH

*Assembly of cytochrome c oxidase in mitochondrial encephalomyopathy-MDA \#228772

2. EMERGENCY: Person(s) designated to handle emergencies

Name: Eric A. Shoubridge Phone No: work: 398-1997 home: $\quad 935-1836$

Name: Tim Johns Phone No: work: $\quad 398-8523$ home: $\quad 488-9771$

3. FUNDING SOURCE OR AGENCY (specify): 228772

Grant No.: New Fund Beginning date:

\begin{tabular}{l} 
MDA, NIH \\
\hline July 1, 2005 \\
July 1, 2005
\end{tabular}

July 1,2005

End date:
June 30,2009

June 30, 2009

4. Indicate if this is

$\bigotimes$ Renewal: procedures previously approved without alterations.

Approval End Date: June 30, 2005

$\bigotimes$ New funding source: project previously reviewed and approved under an application to another agency.
Agency:
N/H
Approval End Date:
June 30, 2005

$\square$ New project: project not previously reviewed.

$\square$ Approved project: change in biohazardous materials or procedures.

$\square$ Work/project involving biohazardous materials in teaching/diagnostics.

CERTIFICATION STATEMENT: Environmental Health \& Safety approves the experimental procedures proposed and certifies with the applicant that the experiment will be in accordance with the principles outlined in Health Canada's

"Laboratory Biosafety Guidelines" and in the "McGill Laboratory Biosafety Manual".

Containment Level (select one): $\quad \square 1 \quad \square_{2}$

Principal Investigator or course director:

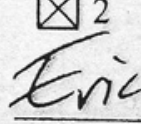

Approved by Environmental Health \& Safety:

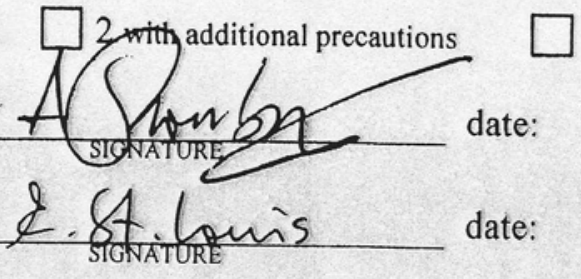

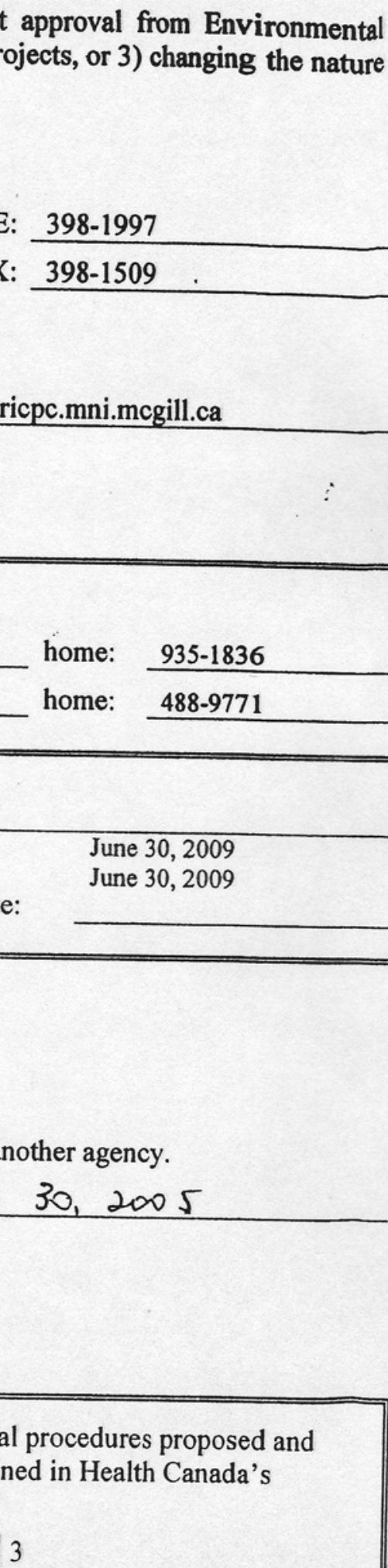

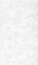


This is a License Agreement between Timothy WAI ("You") and Nature Publishing Group ("Nature Publishing Group") provided by Copyright Clearance Center ("CCC"). The license consists of your order details, the terms and conditions provided by Nature Publishing Group, and the payment terms and conditions.

\section{All payments must be made in full to CCC. For payment instructions, please see information listed at the bottom of this form.}

License Number

License date

Licensed content publisher

Licensed content publication

Licensed content title

Licensed content author

Volume number

Issue number

Pages

Year of publication

Portion used

Requestor type

Type of Use

Billing Type

Company

Billing Address

Customer reference info

Total

0.00 USD

2008

Text

Student

Invoice

Canada
2112620886280

Jan 19, 2009

Nature Publishing Group

Nature Genetics

The mitochondrial DNA genetic bottleneck results from replication of a subpopulation of genomes

Timothy Wai, Daniella Teoli, Eric A Shoubridge

Thesis / Dissertation

Timothy WAI

Montreal Neurological Institute

3801 University Street

Montreal, QC H3A2B4

Terms and Conditions
Terms and Conditions for Permissions 
Nature Publishing Group hereby grants you a non-exclusive license to reproduce this material for this purpose, and for no other use, subject to the conditions below:

1. NPG warrants that it has, to the best of its knowledge, the rights to license reuse of this material. However, you should ensure that the material you are requesting is original to Nature Publishing Group and does not carry the copyright of another entity (as credited in the published version). If the credit line on any part of the material you have requested indicates that it was reprinted or adapted by NPG with permission from another source, then you should also seek permission from that source to reuse the material.

2. Permission granted free of charge for material in print is also usually granted for any electronic version of that work, provided that the material is incidental to the work as a whole and that the electronic version is essentially equivalent to, or substitutes for, the print version. Where print permission has been granted for a fee, separate permission must be obtained for any additional, electronic re-use (unless, as in the case of a full paper, this has already been accounted for during your initial request in the calculation of a print run). NB: In all cases, web-based use of full-text articles must be authorized separately through the 'Use on a Web Site' option when requesting permission.

3. Permission granted for a first edition does not apply to second and subsequent editions and for editions in other languages (except for signatories to the STM Permissions Guidelines, or where the first edition permission was granted for free).

4. Nature Publishing Group's permission must be acknowledged next to the figure, table or abstract in print. In electronic form, this acknowledgement must be visible at the same time as the figure/table/abstract, and must be hyperlinked to the journal's homepage.

5. The credit line should read:

Reprinted by permission from Macmillan Publishers Ltd: [JOURNAL NAME] (reference citation), copyright (year of publication)

For AOP papers, the credit line should read:

Reprinted by permission from Macmillan Publishers Ltd: [JOURNAL NAME], advance online publication, day month year (doi: 10.1038/sj.[JOURNAL ACRONYM].XXXXX)

6. Adaptations of single figures do not require NPG approval. However, the adaptation should be credited as follows:

Adapted by permission from Macmillan Publishers Ltd: [JOURNAL NAME] 
(reference citation), copyright (year of publication)

7. Translations of 401 words up to a whole article require NPG approval. Please visit http://www.macmillanmedicalcommunications.com for more information.

Translations of up to a 400 words do not require NPG approval. The translation should be credited as follows:

Translated by permission from Macmillan Publishers Ltd: [JOURNAL NAME] (reference citation), copyright (year of publication).

We are certain that all parties will benefit from this agreement and wish you the best in the use of this material. Thank you.

v1.1

Gratis licenses (referencing $\$ 0$ in the Total field) are free. Please retain this printable license for your reference. No payment is required.

If you would like to pay for this license now, please remit this license along with your payment made payable to "COPYRIGHT CLEARANCE CENTER" otherwise you will be invoiced within $\mathbf{3 0}$ days of the license date. Payment should be in the form of a check or money order referencing your account number and this license number 2112620886280.

If you would prefer to pay for this license by credit card, please go to http://www.copyright.com/creditcard to download our credit card payment authorization form.

Make Payment To:

Copyright Clearance Center

Dept 001

P.O. Box 843006

Boston, MA 02284-3006

If you find copyrighted material related to this license will not be used and wish to cancel, please contact us referencing this license number 2112620886280 and noting the reason for cancellation.

Questions? customercare@copyright.com or 877-622-5543 or +1-978-646-2777. 